\title{
EXACT RIEMANN SOLUTIONS TO SHALLOW WATER EQUATIONS
}

\author{
BY
}

EE HAN (Institut fuer Analysis und Numerik, Otto-von-Guericke-Universitaet Magdeburg, D-39106 Magdeburg, Germany)

AND

GERALD WARNECKE (Institut fuer Analysis und Numerik, Otto-von-Guericke-Universitaet Magdeburg, D-39106 Magdeburg, Germany)

\begin{abstract}
We determine completely the Riemann solutions to the shallow water equations with a bottom step, including the dry bed problem. The nonstrict hyperbolicity of this first-order system of partial differential equations leads to resonant waves and nonunique solutions. To address these difficulties we construct the $\mathrm{L}-\mathrm{M}$ and $\mathrm{R}-\mathrm{M}$ curves in the state space. For the bottom step elevated from left to right, we classify the L-M curve into five different cases and the $\mathrm{R}-\mathrm{M}$ curve into two different cases based on the subcritical and supercritical Froude number of the Riemann initial data as well as the jump of the bottom step. The behaviors of all basic cases of the $\mathrm{L}-\mathrm{M}$ and $\mathrm{R}-\mathrm{M}$ curves are fully analyzed. We observe that the non-uniqueness of the Riemann solutions is due to bifurcations on the $\mathrm{L}-\mathrm{M}$ or $\mathrm{R}-\mathrm{M}$ curves. The possible solutions including classical waves and resonant waves as well as dry bed state are solved in a uniform framework for any given Riemann initial data.
\end{abstract}

1. Introduction. In this paper we are concerned with the shallow water system of hyperbolic equations, which can be written in the form

$$
\frac{\partial \mathbf{W}}{\partial t}+\frac{\partial \mathbf{F}(\mathbf{W})}{\partial x}=-\mathbf{H}(\mathbf{W}) z_{x}
$$

where

$$
\mathbf{W}=\left[\begin{array}{c}
z \\
h \\
h u
\end{array}\right], \quad \mathbf{F}(\mathbf{W})=\left[\begin{array}{c}
0 \\
h u \\
h u^{2}+g h^{2} / 2
\end{array}\right], \quad \mathbf{H}(\mathbf{W})=\left[\begin{array}{c}
0 \\
0 \\
g h
\end{array}\right]
$$

Received June 12, 2012.

2010 Mathematics Subject Classification. 76B15, 76H05, 35L60.

Key words and phrases. Shock waves, rarefaction waves, velocity function, stationary waves, resonant waves, Froude number, nonuniqueness solutions.

The first author is supported by Micro-Macro-Interactions in structured Media and Particle Systems.

E-mail address: eehan@math.uni-bremen.de

E-mail address: warnecke@ovgu.de 
see, e.g., Stoker [2]. The independent variables $z, h$ and $u$ denote, respectively, the bottom topography, the water height and the water velocity, while $g$ is the gravity constant. Usually the bottom topography $z$ is assumed to be given a priori.

The shallow water equations (1.1) model incompressible flows on a bottom bed under the assumption that the depth of the fluid is much smaller than the wave length of the disturbances considered. It has wide applications in fluid dynamics; for example, tidal flows in an estuary, hydraulic jumps, river beds and channels, tsunamis, etc. The system has also been studied from a mathematical point of view. A particular feature of the system (1.1) is the presence of the bottom topography $z(x)$. This geometric variable is independent of time and leads to a stationary source and a nonconservative term.

We only reference a few publications. LeFloch [18] complemented a related nonconservative system with an additional trivial equation $z_{t}=0$. It introduces a linear degenerate field with a 0 -speed eigenvalue. As a result the system (1.1) becomes a nonstrictly hyperbolic system. Due to the coincidence of eigenvectors the system becomes degenerate at sonic states; see, e.g., Alcrudo and Benkhaldoun [5]. Bernettia et al. [7] studied an enlarged system and used the energy to rule out solutions that are physically inadmissible. Andrianov [3] proposed an example which has two solutions for one set of initial data to show that different numerical schemes may approach different exact solutions. Li and Chen [10] studied the generalized Riemann problem for the current system. LeFloch and Thanh in [19,20] investigated the exact Riemann solutions. They obtained most of the possible solutions for given initial data. However, they omitted one possible type of solution, which is denoted as the wave configuration $E$ in this work. Moreover, they did not give complete proofs for the existence and uniqueness of the solutions, especially for the conjecture in [20, Remark 6, p. 7646]. Besides the papers we just mentioned, considerable work has been devoted to the topic of the shallow water equations; see, e.g., 1, 2, 12, 14 and the references therein.

In this work we propose a uniform framework to solve the system (1.1) with the following Riemann initial data

$$
\mathbf{W}(x, 0)= \begin{cases}\mathbf{W}_{L}, & x<x_{0}, \\ \mathbf{W}_{R}, & x>x_{0},\end{cases}
$$

where $\mathbf{W}_{q}$ with $q=L$ or $R$ are constant. There are three wave curves for the system (1.1). The first and second wave curves are given by the physically relevant parts of the rarefaction and shock curves. The third wave curve, denoted as the stationary wave curve, is due to the variation of the bottom step. Since the governing system is nonstrictly hyperbolic, the mutual positions of the stationary wave curve with respect to the rest of the two elementary waves cannot be determined a priori. To address this difficulty, we introduce the $\mathrm{L}-\mathrm{M}$ and $\mathrm{R}-\mathrm{M}$ curves in the state plane for the construction of solutions to Riemann problems. The idea is motivated by Marchesin and Paes-Leme 21] as well as our previous work for the exact Riemann solutions to Euler equations in ducts with discontinuous cross-section in [9].

During this work we always assume without loss of generality that $z_{L}<z_{R}$. The opposite case can be treated as the mirror-image problem by reflecting the Riemann initial data in terms of $x=x_{0}$ and setting the velocities in the opposite direction. We 
take into account the stationary wave curves by deriving a velocity function. Owing to this function, the $\mathrm{L}-\mathrm{M}$ and $\mathrm{R}-\mathrm{M}$ curves with $z_{L}<z_{R}$ can be, respectively, classified into five and two different cases by the subcritical or supercritical Froude number of the Riemann initial data as well as the jump of the bottom step. This new classification is very helpful for a systematic consideration of solutions. It is given for the $\mathrm{L}-\mathrm{M}$ curves at the beginning of Section 4.2 and for the $\mathrm{R}-\mathrm{M}$ curves in Section 4.3 . Note that each of these curves leads to more than one wave configuration, depending on the Riemann initial data. We obtain the 7 wave configurations, denoted as $A, B, C, D, E, F, G$, that do not have a dry bed state and 6 that do have a dry bed state in the solution. The dry bed states are like vacuum states in gas dynamics and therefore we index the corresponding wave configurations with subscript letter $v$.

We find that the water can always spread across a lowered bottom step. But the water can go across an elevated bottom step if and only if a critical step size $z_{\max }$ is larger than the actual jump height of the bottom step. The critical step size $z_{\text {max }}$ is determined by the height and Froude number of the inflow state.

We carefully study the monotonicity and smoothness properties of the $\mathrm{L}-\mathrm{M}$ and $\mathrm{R}-$ $\mathrm{M}$ curves in each case. Note that the introduction of these curves and the use of the velocity function make our approach to the solution of the Riemann problem different from the previous work. We feel that this makes the solution procedure clearer and simpler. Observe that a bifurcation occurs for certain cases. This bifurcation introduces nonunique solutions and validates the conjecture in [20, Remark 6, p. 7646]. Specifically, we solve the dry bed problem of the solution in this framework. Here the dry bed problem refers two subcases. One is for the water propagating to a dry bed; see Toro [1]. The other one is for the dry bed state emerging due to the motion of the flow.

The organization of the paper is as follows. We briefly review the fundamental concepts and notions for the governing system in Section 2. In Section 3 we discuss the stationary wave curves. Our main focus is in Section 4, which contains the definition of the $\mathrm{L}-\mathrm{M}$ and $\mathrm{R}-\mathrm{M}$ curves and the complete analysis of their structures. All the possible wave configurations are illustrated in this section. The algorithm for determining the exact solutions is explained in Section 5. Finally we make some conclusions in Section 6.

2. The shallow water system. We now derive the quasilinear form of the system (1.1). Set $\mathbf{V}=(z, h, u)^{T}$; then

$$
\mathbf{V}_{t}+\mathbf{A}(\mathbf{V}) \mathbf{V}_{x}=0
$$

where the Jacobian matrix $\mathbf{A}(\mathbf{V})$ is in the form

$$
\mathbf{A}(\mathbf{V})=\left(\begin{array}{lll}
0 & 0 & 0 \\
0 & u & h \\
g & g & u
\end{array}\right)
$$

The eigenvalues of $\mathbf{A}(\mathbf{V})$ are

$$
\lambda_{0}=0, \quad \lambda_{1}=u-c, \quad \lambda_{2}=u+c,
$$

where $c=\sqrt{g h}$ is the sound speed. The eigenvalues are referred to as the characteristic speeds. The system (1.1) is not strictly hyperbolic as a result of the fact that $\lambda_{0}$ can 
coincide with any of the two other eigenvalues. The corresponding right eigenvectors are

$$
\mathbf{R}_{0}=\left(\begin{array}{c}
\frac{c^{2}-u^{2}}{c^{2}} \\
1 \\
-\frac{u}{h}
\end{array}\right), \quad \mathbf{R}_{1}=\left(\begin{array}{c}
0 \\
1 \\
-\frac{c}{h}
\end{array}\right), \quad \mathbf{R}_{3}=\left(\begin{array}{c}
0 \\
1 \\
\frac{c}{h}
\end{array}\right)
$$

One can easily show that

$$
\mathbf{R}_{0} \rightarrow \mathbf{R}_{k} \quad \text { as } \quad \lambda_{k} \rightarrow 0 \quad \text { for } \quad k=1,2 .
$$

Consequently the system (1.1) is degenerate for the states at which the eigenvalues $\lambda_{1}$ or $\lambda_{2}$ coincide with $\lambda_{0}$. Specifically, this state is the sonic state at which $u= \pm c$.

We use the terminology $k$-waves, $k=0,1,2$, to denote the waves associated to the $k$-characteristic fields when the eigenvalues are distinct from each other. Here the 1and 2-waves are shocks (hydraulic jumps) or rarefactions. Traditionally the 0 -wave is named the stationary wave due to the jump of the bottom step. Note that a 0 -speed shock or a transonic rarefaction wave will coincide with the stationary wave. In such a case these elementary waves will be involved in the stationary wave [15]. We name these combined waves as the resonant waves. They will be studied in detail later; see also Han et al. 9].

We define the shock and rarefaction curves. Let $\mathbf{U}_{q}=\left(h_{q}, u_{q}\right)^{T}$ be any state in the state space. The shock speed $\sigma_{k}$ and the velocity $u$ can be expressed as follows:

$$
\begin{aligned}
\sigma_{k}\left(h ; \mathbf{U}_{q}\right) & =u_{q} \pm h \sqrt{\frac{g}{2}\left(\frac{1}{h}+\frac{1}{h_{q}}\right)}, \\
u & =u_{q} \pm\left(h-h_{q}\right) \sqrt{\frac{g}{2}\left(\frac{1}{h}+\frac{1}{h_{q}}\right)},
\end{aligned}
$$

where $h>h_{q}$ and the subscript $k=1,2$ represents the number of the wave family. The sign - is taken when $k=1$ while the sign + is taken when $k=2$. The detailed derivation can be found in Francisco and Benkhaldoun [5]. The admissible shock curves $S_{k}\left(\mathbf{U}_{q}\right)$, $k=1,2$ denote the states which are connected to the state $\mathbf{U}_{q}$ by an admissible 1 -shock or 2 -shock respectively. Set

$$
S_{k}\left(\mathbf{U}_{q}\right)=\left\{(h, u) \mid u=u_{q} \pm\left(h-h_{q}\right) \sqrt{\frac{g}{2}\left(\frac{1}{h}+\frac{1}{h_{q}}\right)} \text { with } h>h_{q}\right\}, k=1,2 .
$$

Generally the shock curve $S_{k}\left(\mathbf{U}_{q}\right)$ contains three components, namely,

$$
\begin{aligned}
S_{k}^{ \pm}\left(\mathbf{U}_{q}\right) & =\left\{(h, u) \mid(h, u) \in S_{k}\left(\mathbf{U}_{q}\right) \text { and } \sigma_{k}\left(h ; \mathbf{U}_{q}\right) \gtrless 0\right\}, \\
S_{k}^{0}\left(\mathbf{U}_{q}\right) & =\left\{(h, u) \mid(h, u) \in S_{k}\left(\mathbf{U}_{q}\right) \text { and } \sigma_{k}\left(h ; \mathbf{U}_{q}\right)=0\right\} .
\end{aligned}
$$

We study the state set $S_{k}^{0}\left(\mathbf{U}_{q}\right)$. Note that we have the shock speed

$$
\sigma_{k}\left(h ; \mathbf{U}_{q}\right)=u_{q} \pm h \sqrt{\frac{g}{2}\left(\frac{1}{h}+\frac{1}{h_{q}}\right)}=0 \quad \text { with } \quad h>h_{q} .
$$


Therefore, introducing the Froude number $F_{q}:=\frac{u_{q}}{c_{q}}=\frac{u_{q}}{\sqrt{g h_{q}}}$, we obtain

$$
\left(\frac{h}{h_{q}}\right)^{2}+\frac{h}{h_{q}}-2 F_{q}^{2}=0 .
$$

There are two solutions to (2.10) which are

$$
h_{1}=\frac{-1+\sqrt{1+8 F_{q}^{2}}}{2} h_{q}, \quad h_{2}=\frac{-1-\sqrt{1+8 F_{q}^{2}}}{2} h_{q} .
$$

Note that $h_{1}>h_{q}$ and $h_{2}<0<h_{q}$, so $h_{1}$ is the physically relevant solution to (2.9). Hence the set $S_{k}^{0}\left(\mathbf{U}_{q}\right)$ contains only one state. Hereafter we use $\hat{\mathbf{U}}_{q}=S_{k}^{0}\left(\mathbf{U}_{q}\right)$ to denote it; then we have

$$
\hat{h}_{q}=\frac{-1+\sqrt{1+8 F_{q}^{2}}}{2} h_{q} .
$$

Since $\hat{h}_{q} \hat{u}_{q}=h_{q} u_{q}$, we get $\hat{u}_{q}=\frac{h_{q} u_{q}}{\hat{h}_{q}}$. Direct calculation yields

$$
\hat{u}_{q}=\frac{1+\sqrt{1+8 F_{q}^{2}}}{4 F_{q}^{2}} u_{q} .
$$

For the rarefaction curves, similarly, we use $R_{k}\left(\mathbf{U}_{q}\right)$ to denote the states $\mathbf{U}$ which can be connected to $\mathbf{U}_{q}$ by a $k$-rarefaction wave, i. e.

$$
R_{k}\left(\mathbf{U}_{q}\right)=\left\{(h, u) \mid u=u_{q} \pm 2\left(c-c_{q}\right) \text { with } h \leq h_{q}\right\}, k=1,2 .
$$

2.1. The 1-wave and 2-wave curves. Generally the $k$-wave curves $T_{k}\left(\mathbf{U}_{q}\right), k=1,2$, are defined as the sets of states which can be connected to the initial state $\mathbf{U}_{q}$ by admissible waves. That is to say we have

$$
T_{1}\left(\mathbf{U}_{L}\right)=R_{1}\left(\mathbf{U}_{L}\right) \cup S_{1}\left(\mathbf{U}_{L}\right), \quad T_{2}\left(\mathbf{U}_{R}\right)=R_{2}\left(\mathbf{U}_{R}\right) \cup S_{2}\left(\mathbf{U}_{R}\right) .
$$

Obviously $T_{1}\left(\mathbf{U}_{L}\right)$ and $T_{2}\left(\mathbf{U}_{R}\right)$ are the admissible wave curves associated to the characteristic field with $\lambda_{1}$ and $\lambda_{2}$ in the state space respectively.

For simplicity we define the following function

$$
f\left(h ; h_{q}\right):= \begin{cases}2\left(\sqrt{g h}-c_{q}\right), & \text { if } h \leq h_{q}, \\ \left(h-h_{q}\right) \sqrt{\frac{g}{2}\left(\frac{1}{h}+\frac{1}{h_{q}}\right)}, & \text { if } h>h_{q} .\end{cases}
$$

We will consider $f\left(h ; h_{q}\right)$ as a function of $h$ for given parameter $h_{q}$. Therefore the $k$-wave curve $T_{k}\left(\mathbf{U}_{q}\right), k=1,2$ can be rewritten as

$$
\begin{aligned}
& T_{1}\left(\mathbf{U}_{q}\right)=\left\{\mathbf{U} \mid u=u_{q}-f\left(h ; h_{q}\right), h \geq 0\right\}, \\
& T_{2}\left(\mathbf{U}_{q}\right)=\left\{\mathbf{U} \mid u=u_{q}+f\left(h ; h_{q}\right), h \geq 0\right\} .
\end{aligned}
$$

Lemma 2.1. The function $f\left(h ; h_{q}\right)$ is continuously differentiable, strictly increasing and concave.

Proof. The function $f\left(h ; h_{q}\right)$ is twice continuous due to $\lim _{h \rightarrow h_{q}-} f\left(h ; h_{q}\right)=$ $\lim _{h \rightarrow h_{q}+} f\left(h ; h_{q}\right)=0$. The derivative of the function $f\left(h ; \mathbf{Q}_{q}\right)$ is

$$
f^{\prime}\left(h ; h_{q}\right):= \begin{cases}\sqrt{\frac{g}{h}}, & \text { if } h \leq h_{q}, \\ \sqrt{\frac{g}{2}} \frac{\frac{1}{h}+\frac{2}{h_{q}}+\frac{h_{q}}{h^{2}}}{2 \sqrt{\frac{1}{h}+\frac{1}{h_{q}}}} & \text { if } h>h_{q} .\end{cases}
$$


Therefore we have

$$
f^{\prime}\left(h ; h_{q}\right)>0
$$

and $\lim _{h \rightarrow h_{q}} f^{\prime}\left(h ; h_{q}\right)=\sqrt{\frac{g}{h_{q}}}$. To see the convexity of the function, we need to consider the second derivative of the function $f\left(h ; h_{q}\right)$. Actually we have

$$
f^{\prime \prime}\left(h ; h_{q}\right):= \begin{cases}-\frac{1}{2} \sqrt{g} h^{-\frac{3}{2}}, & \text { if } h \leq h_{q}, \\ -\frac{\sqrt{g}}{4 \sqrt{2}} \frac{\frac{5}{h^{3}}+\frac{3 h_{q}}{h^{4}}}{\left(\frac{1}{h}+\frac{1}{h_{q}}\right)^{\frac{3}{2}}}, & \text { if } h>h_{q} .\end{cases}
$$

It follows that $f^{\prime \prime}\left(h ; h_{q}\right)<0$. Moreover, we have $\lim _{h \rightarrow h_{q}} f^{\prime \prime}\left(h ; h_{q}\right)=-\frac{1}{2} \sqrt{g} h_{q}^{-\frac{3}{2}}$. This is enough to confirm the lemma.

Lemma 2.1 reveals that the 1 -wave curve $T_{1}\left(\mathbf{U}_{L}\right)$ is a strictly decreasing concave curve, while the 2 -wave curve $T_{2}\left(\mathbf{U}_{R}\right)$ is a strictly increasing convex curve in the $(u, h)$ state plane. Therefore these two curves have at most one intersection point. To find whether the intersection point exists, we need to consider the state with $h=0$, which corresponds to the dry bed of the water; see Toro [11. For the 1-wave curve $T_{1}\left(\mathbf{U}_{L}\right)$ and the 2 -wave curve $T_{2}\left(\mathbf{U}_{R}\right)$ we take $h=0$ in (2.17) and (2.16). We obtain two velocities

$$
u_{0 L}=u_{L}+2 c_{L}
$$

and

$$
u_{0 R}=u_{R}-2 c_{R} .
$$

These are the velocities of the water covering or uncovering a dry state $h=0$. The two curves $T_{1}\left(\mathbf{U}_{L}\right)$ and $T_{2}\left(\mathbf{U}_{R}\right)$ will interact if $u_{0 L} \geq u_{0 R}$, i.e.

$$
u_{R}-u_{L} \leq 2\left(c_{L}+c_{R}\right) \text {. }
$$

In this case the intersection point of $T_{1}\left(\mathbf{U}_{L}\right)$ and $T_{2}\left(\mathbf{U}_{R}\right)$ uniquely exists. Otherwise, if $u_{0 L}<u_{0 R}$, there is no intersection point. The solution involves a dry bed intermediate state.

Next we turn to study two specific dry bed problems. Both of them concern the water receding from the jump of the dry bed. The first problem has the Riemann initial data

$$
(h, u)(x, 0)= \begin{cases}\left(h_{L}, u_{L}\right), & x<0 \\ (0,0), & x>0\end{cases}
$$

with the restriction that $u_{0 L}<0$. In such a case the 2 -wave of the solution is missing while the 1 -wave is a rarefaction wave on the left side. The corresponding solution is given as

$$
(h, u)(x, t)= \begin{cases}\left(h_{L}, u_{L}\right), & \frac{x}{t} \leq u_{L}-c_{L} \\ \left(\frac{\left(u_{L}+2 c_{L}-\frac{x}{t}\right)^{2}}{9 g}, \frac{u_{L}+2 c_{L}+2 \frac{x}{t}}{3}\right), & u_{L}-c_{L}<\frac{x}{t}<u_{0 L}, \\ (0,0), & \frac{x}{t}>u_{0 L} .\end{cases}
$$

The other problem has the Riemann initial data

$$
(h, u)(x, 0)= \begin{cases}(0,0), & x<0, \\ \left(h_{R}, u_{R}\right), & x>0,\end{cases}
$$


with $u_{0 R}>0$. Similarly, the 1 -wave of the solution is missing and the $2-$ wave is a rarefaction wave on the right side. The exact solution of this case is shown in the following:

$$
(h, u)(x, t)= \begin{cases}\left(h_{R}, u_{R}\right), & \frac{x}{t} \geq u_{R}+c_{R}, \\ \left(\frac{\left(u_{R}-2 c_{R}-\frac{x}{t}\right)^{2}}{9 g}, \frac{u_{R}-2 c_{R}+2 \frac{x}{t}}{3}\right), & u_{R}+c_{R}>\frac{x}{t} \geq u_{0 R}, \\ (0,0), & \frac{x}{t}<u_{0 R} .\end{cases}
$$

The jump of the bottom step does not affect the solution in these two examples. However for the Riemann problem (1.1), (2.24) or (2.26) but with $u_{0 L}>0$ or $u_{0 R}>0$ respectively, the jump of the bottom step induces an additional wave. The motion of the flow becomes more complicated, not to mention the general Riemann problem of (1.1) and (1.3) with $h_{L}>0$ and $h_{R}>0$. There the jump of the bottom step greatly affects the motion of the flow. So, in the next section, we study the stationary wave due to the jump of the bottom step.

3. The stationary wave curve. The stationary wave curve for the system (1.1) is defined by the ODE system

$$
\frac{\partial \mathbf{F}(\mathbf{U})}{\partial x}=-\mathbf{H}(\mathbf{U}) z_{x}
$$

Motivated by Alcrudo and Benkhaldoun [5] and references cited therein, we have the following Lemma.

Lemma 3.1. For the smooth bottom topography the sonic state can only appear when the bottom function reaches a maximum.

Proof. The ODE system (3.1) asserts the following equations

$$
\begin{aligned}
\frac{\partial h u}{\partial x} & =0 \\
u \frac{\partial u}{\partial x}+g \frac{\partial h+z}{\partial x} & =0
\end{aligned}
$$

Therefore we have

$$
\left(1-\frac{u^{2}}{c^{2}}\right) \frac{h}{u} u_{x}=z_{x}
$$

The relation (3.3) shows that for smooth lowered bottom topography, i.e. $z_{x}<0$, the velocity of the water decreases when $u^{2}<c^{2}$ and vice versa. Similarly for smooth elevated bottom topography, i.e. $z_{x}>0$, the velocity increases when $u^{2}<c^{2}$ and vice versa. So we can conclude that the quantity $z$ as a function of $x$ has a maximum at the sonic state $u^{2}=c^{2}$.

In this work we regard the stationary wave as a transition layer located at $x=0$ with 0 width. In this approach the discontinuous variation of the bottom step is viewed as the limiting case of locally monotonic bottom slope going to infinity. This idea has been used by Alcrudo and Benkhaldoun [5], LeFloch and Thanh [19,20], Toro [11] etc. for the shallow water systems. Han et. al. 9] also adopted it to solve the Riemann problem for duct flows. 
3.1. The stationary wave. In this section we use the subscript $i$ to signify the inflow variables and $o$ to signify the outflow variables. Assume that the piecewise constant bottom topography has the values $z_{i}$ and $z_{o}$, while the upstream flow state is $\left(h_{i}, u_{i}\right)$ (which is known) and the downstream flow state is $(h, u)$. Here $z_{i}$ will be $z_{L}$ if $u>0$, while it is $z_{R}$ if $u<0$. With the analogous consideration $z_{o}$ will be determined.

Let us assume that $h_{i}, h>0$. One can easily derive the following relations from the system (3.1)

$$
\begin{aligned}
h u & =h_{i} u_{i}, \\
\frac{u^{2}}{2}+g\left(h+z_{o}\right) & =\frac{u_{i}^{2}}{2}+g\left(h_{i}+z_{i}\right) .
\end{aligned}
$$

The formula (3.4) implies the following conditions:

(1) $u_{i}$ and $u$ have the same sign,

(2) $u_{i}=0 \Longleftrightarrow u=0$.

Our aim is to calculate the downstream state $(h, u)$. Specifically if $u_{i}=0$ and $h_{i}+z_{i}-z_{o}>$ 0 , we have $u=0$ and $h=h_{i}+z_{i}-z_{o}$. Otherwise if $u_{i}=0$ and $h_{i}+z_{i}-z_{o}<0$, we have $u=0$ and $h=0$. In the following analysis we always assume that $u_{i} \neq 0$. For simplicity we can use the notation $\mathbf{U}=\mathbf{J}\left(z_{o} ; \mathbf{U}_{i}, z_{i}\right)$ to represent the explicit solution $\mathbf{U}:=(h, u)$ implicitly given by (3.4) and (3.5). Our aim is to calculate the downstream state $(h, u)$ of the flow for the known upstream flow $\left(h_{i}, u_{i}\right)$. A velocity function is derived from (3.4) and (3.5) to be

$$
\Psi\left(u ; \mathbf{U}_{i}, z_{i}, z_{o}\right):=\frac{u^{2}}{2}+\frac{c_{i}^{2} u_{i}}{u}-\frac{u_{i}^{2}}{2}-g h_{i}+g\left(z_{o}-z_{i}\right) .
$$

The behavior of the velocity function is analyzed in the following lemma.

Lemma 3.2. Consider

$$
u^{*}=\left(u_{i} c_{i}^{2}\right)^{\frac{1}{3}} .
$$

Then the velocity function $\Psi\left(u ; \mathbf{U}_{i}, z_{i}, z_{o}\right)$ has the following properties:

(1) $\Psi\left(u ; \mathbf{U}_{i}, z_{i}, z_{o}\right)$ decreases if $u<u^{*}$;

(2) $\Psi\left(u ; \mathbf{U}_{i}, z_{i}, z_{o}\right)$ increases if $u>u^{*}$;

(3) $\Psi\left(u ; \mathbf{U}_{i}, z_{i}, z_{o}\right)$ has the minimum value at $u=u^{*}$. Moreover, we have $\left(u^{*}\right)^{2}=$ $\left(c^{*}\right)^{2}$.

Proof. The velocity function $\Psi\left(u ; \mathbf{U}_{i}, z_{o}\right)$ is smooth since if $u_{i}>0$ the existence region for $u$ is $u>0$,. Otherwise if $u_{i}<0$ the existence region for $u$ is $u<0$. Therefore the derivative of $\Psi\left(u ; \mathbf{U}_{i}, z_{i}, z_{o}\right)$ is

$$
\frac{\partial \Psi\left(u ; \mathbf{U}_{i}, z_{i}, z_{o}\right)}{\partial u}=u-\frac{u_{i} c_{i}^{2}}{u^{2}}
$$

Consequently we get

$$
\frac{\partial \Psi\left(u ; \mathbf{U}_{i}, z_{i}, z_{o}\right)}{\partial u} \begin{cases}<0, & \text { if } u<u^{*}, \\ =0, & \text { if } u=u^{*}, \\ >0, & \text { if } u>u^{*}\end{cases}
$$

It follows that the velocity function $\Psi\left(u ; \mathbf{U}_{i}, z_{i}, z_{o}\right)$ is decreasing when $u<u^{*}$, increasing when $u>u^{*}$ and has the minimum value at $u=u^{*}$. 
Since

$$
c^{2}=g h=\frac{g h_{i} u_{i}}{u}
$$

we get the formula

$$
u \frac{\partial \Psi}{\partial u}\left(u ; \mathbf{U}_{i}, z_{i}, z_{o}\right)=u^{2}-\frac{g u_{-} h_{-}}{u}=u^{2}-c^{2} .
$$

From $\frac{\partial \Psi\left(u^{*} ; \mathbf{U}_{i}, z_{i}, z_{o}\right)}{\partial u}=0$ we obtain $\left(u^{*}\right)^{2}=\left(c^{*}\right)^{2}$.

REMARK 3.3. Lemma 3.2 shows that the equation $\Psi\left(u ; \mathbf{U}_{i}, z_{i}, z_{o}\right)=0$ may have two, one or no solutions. Further discussions are as follows.

(1) If the minimum value $\Psi\left(u^{*} ; \mathbf{U}_{i}, z_{i}, z_{o}\right)<0$, the equation $\Psi\left(u ; \mathbf{U}_{i}, z_{i}, z_{o}\right)=0$ has two roots. Assume that the root closer to 0 is $u_{l}$ and the other one is $u_{r}$, with $c_{l}$ and $c_{r}$ being the corresponding sound speeds. Then according to (3.11), $u_{l}^{2}-c_{l}^{2}<0$ and $u_{r}^{2}-c_{r}^{2}>0$. It is well known that the transition from subcritical to supercritical channel flow can only occur at points of maximum of the bottom function [5]. So physically we can take the one which satisfies

$$
\operatorname{sign}\left(u_{q}^{2}-c_{q}^{2}\right)=\operatorname{sign}\left(u_{i}^{2}-c_{i}^{2}\right)
$$

where $q=l$ or $r$. However, one special case is that if the inflow state $\mathbf{U}_{i}$ is a sonic state, i.e. $u_{i}^{2}=c_{i}^{2}$, then (3.12) no longer holds. There are two possible solutions $u_{l}$ and $u_{r}$ to the corresponding velocity function (3.6); which one is to be chosen depends on the requirement of the specific problem. The details will be given later.

(2) If $\Psi\left(u^{*} ; \mathbf{U}_{i}, z_{i}, z_{o}\right)=0$, the equation $\Psi\left(u ; \mathbf{U}_{i}, z_{i}, z_{o}\right)=0$ has exactly one solution which is the sonic state, i.e. $u=u^{*}$.

(3) If $\Psi\left(u^{*} ; \mathbf{U}_{i}, z_{i}, z_{o}\right)>0$, the equation $\Psi\left(u ; \mathbf{U}_{i}, z_{i}, z_{o}\right)=0$ has no solution.

The procedure for calculating the outflow state $\mathbf{U}=\mathbf{J}\left(z_{o} ; \mathbf{U}_{i}, z_{i}\right)$ is summarized in Algorithm 1] However it is necessary to analyze the existence region for $\mathbf{U}=\mathbf{J}\left(z_{o} ; \mathbf{U}_{i}, z_{i}\right)$ to determine it a priori.

3.2. Existence of the stationary wave. Remark 3.3 reveals that the velocity function may have no solutions. To be more precise, we now consider the existence conditions for the outflow state $(h, u)$ of the stationary wave introduced above. According to Lemma 3.2. it is equivalent to evaluate the minimum value of the velocity function $\Psi\left(u ; \mathbf{U}_{i}, z_{i}, z_{o}\right)$ being not larger than 0, i.e.

$$
\Psi\left(u^{*} ; \mathbf{U}_{i}, z_{i}, z_{o}\right)=\frac{3}{2}\left(u_{i} c_{i}^{2}\right)^{\frac{2}{3}}-c_{i}^{2}-\frac{u_{i}^{2}}{2}+g\left(z_{o}-z_{i}\right) \leq 0 .
$$

We introduce the Froude number $F_{i}:=\frac{u_{i}}{c_{i}}$; then we have

$$
h_{i}\left(\frac{3}{2}\left(F_{i}\right)^{\frac{2}{3}}-\frac{F_{i}^{2}}{2}-1\right)+z_{o}-z_{i} \leq 0 .
$$

Therefore we obtain

We know that

$$
z_{o}-z_{i} \leq h_{i}\left(\frac{F_{i}^{2}}{2}-\frac{3}{2} F_{i}^{\frac{2}{3}}+1\right) .
$$

$$
\frac{F_{i}^{2}}{2}-\frac{3}{2} F_{i}^{\frac{2}{3}}+1 \geq 0
$$




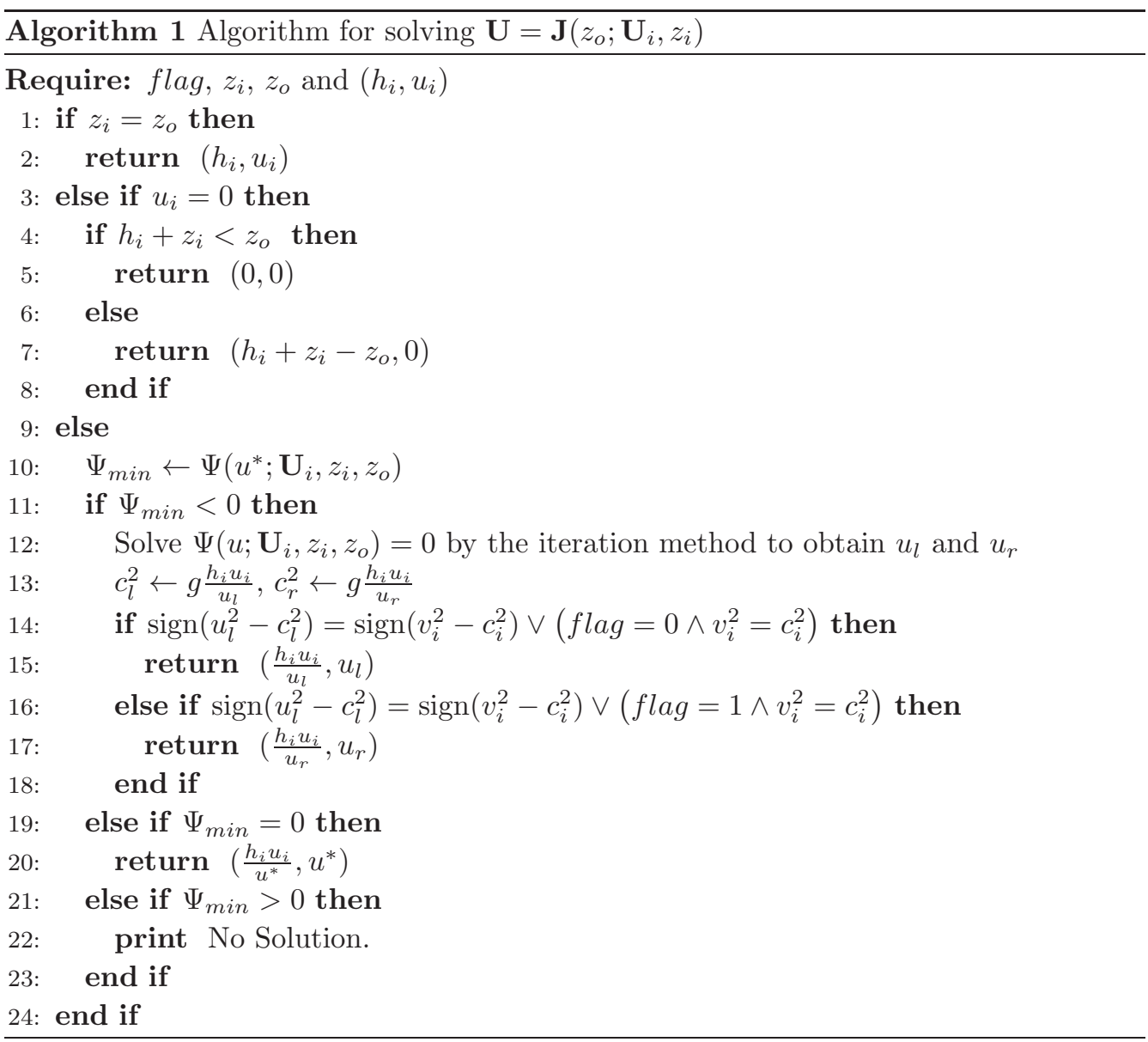

It reaches 0 if and only if $F_{i}=1$. Hence, the above computation motivates the following theorem.

TheOREM 3.4. The existence of the solution to the velocity function.

(1) If $z_{o}<z_{i}, \Psi\left(u ; \mathbf{U}_{i}, z_{i}, z_{o}\right)$ always has solutions.

(2) Otherwise if $z_{o}>z_{i}, \Psi\left(u ; \mathbf{U}_{i}, z_{i}, z_{o}\right)$ has a solution if and only if

$$
z_{o}-z_{i} \leq h_{i}\left(\frac{F_{i}^{2}}{2}-\frac{3}{2} F_{i}^{\frac{2}{3}}+1\right) .
$$

Theorem 3.4 indicates that on one hand the water can always spread across the lowered jump of the bottom step; on the other hand, the water can overflow the elevated jump of the bottom step if and only if the bottom step is not too high. Specifically it should be less than a critical value which is determined by the height and the Froude number of the inflow.

REMARK 3.5. For the fixed inflow state $\mathbf{U}_{i}$ and two outflow bottom steps $z_{o}^{1}<z_{o}^{2}$, if $\mathbf{J}\left(z_{o}^{2} ; \mathbf{U}_{i}, z_{i}\right)$ exists, then $\mathbf{J}\left(z_{o}^{1} ; \mathbf{U}_{i}, z_{i}\right)$ also exists. Since we regard the discontinuous 


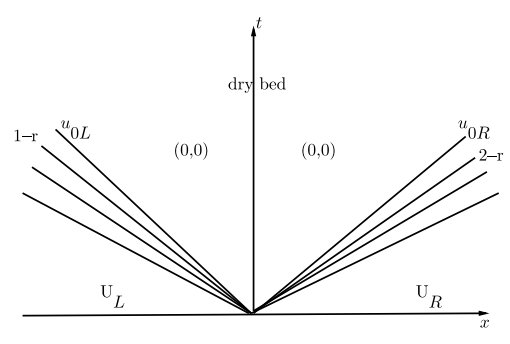

FIG. 1. $A_{v}$

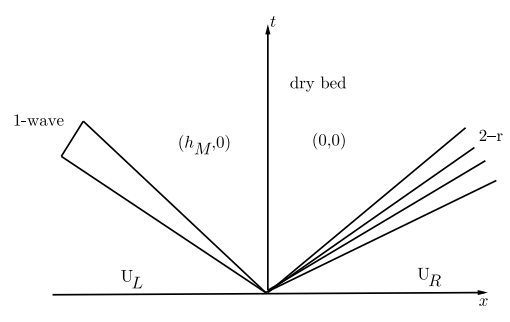

FIG. 3. $H_{1}$



FIG. 2. $A$

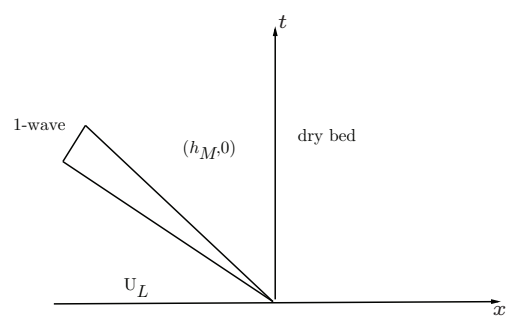

FIG. 4. $H_{2}$

bottom step as the limiting case of monotonic bottom step, it make sense to assume that the solution inside this transition layer is also continuous if there is no resonant wave.

4. $\mathbf{L}-\mathbf{M}$ and $\mathbf{R}-\mathbf{M}$ wave curves. In this work we always assume without loss of generality that

$$
z_{L}<z_{R}
$$

According to Lemma 3.1. the sonic state can only be located on the side $z=z_{R}$ of the stationary wave. The opposite case $z_{L}>z_{R}$ can be treated as the mirror-image problem by reversing the Riemann initial data and setting the velocity in the opposite direction.

Here we study the general Riemann solution which contains a stationary wave. The sufficient condition for this requirement is that $u_{0 L}>0$ or $u_{0 R}<0$, where $u_{0 L}$ and $u_{0 R}$ were defined in (2.21) and (2.22). Otherwise if $u_{0 L}<0$ and $u_{0 R}>0$ the dry bed appears around the initial discontinuity point $x=x_{0}$. Specifically the solution has the wave configuration $A_{v}$; see Figure 1. Hereafter the symbols $k-\mathrm{r}, k=1,2$ denote the $k$-rarefactions. An example of this case can be found in Figure 5 . We can see that the jump of the bottom does not affect the motion of the flow. Therefore there is no stationary wave.

The general exact Riemann solution for the system (1.1) with (1.3) with $u_{0 L}>0$ or $u_{0 R}<0$ consists of a stationary wave which is located at $x=0$ as well as a sequence of 1 - and 2-shocks or rarefactions. Alcrudo and Benkhaldoun in [5] presented more than 20 different solution patterns. Indeed, the solution patterns without the dry bed under the condition (4.1) can be classified into 10 different wave configurations. We show them in Figures 2, 6, 8, 10, 12, 9, 14, 16, 17, and 18, In all of the wave configurations, the 1 - and 2-wave represent a shock or a rarefaction. The dashed right arrow indicates that 

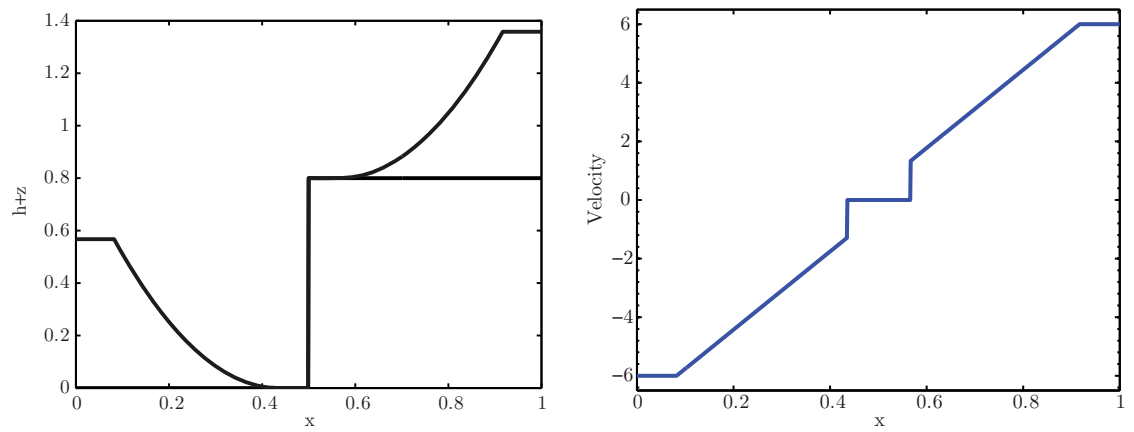

Fig. 5. Left: The water free surface $h+z$ at $t=0.05$; Right: The velocity. The Riemann initial data are $\left(z_{L}, h_{L}, u_{L}\right)=$ $(0.0,0.5674,-6.0)$ when $x<0.5$ and $\left(z_{R}, h_{R}, u_{R}\right)=(0.8,0.558,6.0)$ when $x>0$.

the velocity across the bottom jump is positive, while the dashed left arrow indicates that the velocity across the bottom jump is negative. Note that the wave configuration $E$ has been omitted by LeFloch and Thanh in [20].

The wave configurations $A^{T}, C^{T}$ and $D^{T}$, in some sense, can be viewed as the imagereflection of the wave configurations $A, C$ and $D$ in terms of $x=0$ respectively. Moreover, the wave configurations $B$ and $G$ contain a resonant wave due to the coincidence of the stationary wave with a 1 - and 2 -rarefaction wave respectively. The wave configurations $C$ and $C^{T}$ result from the coincidence of a stationary wave with a 0 -speed $1-$ and $2-$ shock wave respectively, while the wave configurations $E$ and $F$ are the combination of a transonic rarefaction, a stationary wave and a 0 -speed shock. We point out that analogous resonant waves to these mentioned here for other systems can be found in Goatin and LeFloch [15, Rochette and Clain [17, Han et al. 9] etc.

The solution patterns with a dry bed consist of the wave configurations $A_{v}, H_{1}$ and $H_{2}$; see Figures 1 [3, and 4 respectively. Also the wave configuration $B_{v}$, see Figure 7 , belongs to this category. Note that the wave configuration $B_{v}$ originated from the wave configuration $B$. But $B_{v}$ contains a dry bed intermediate state $(0,0)$, and the $2-$ wave is a rarefaction wave. Here we should keep in mind that a 2-rarefaction wave will totally disappear if $h_{R}=0$. This is analogous to the wave configurations $D_{v}$ and $E_{v}$; see Figures 11 and 13 which comes from the wave configurations $D$ and $E$ respectively. The wave configuration $G_{v}$, see Figure 15, originated from the wave configuration $G$. Be advised that $G_{v}$ contains a dry bed state $(0,0)$ and a 1 -rarefaction if $h_{L}>0$, or no 1-wave if $h_{L}=0$. The situation for the wave configuration $D_{v}^{T}$, see Figure 19, is similar.

For one given set of initial data we cannot determine the wave configuration of the solution from the initial data in advance due to many possibilities of the mutual position between the stationary wave and shocks or rarefactions. This is the nature of a nonstrictly hyperbolic system. Analogous to the Euler equations in a duct, see Han et al. 9], we again introduce the $\mathrm{L}-\mathrm{M}$ and $\mathrm{R}-\mathrm{M}$ curves to solve this problem. We merge the stationary wave curve into the 1 -wave curve $T_{1}\left(\mathbf{U}_{L}\right)$ or the 2 -wave curve $T_{2}\left(\mathbf{U}_{R}\right)$. Here we also name them $\mathrm{L}-\mathrm{M}$ and $\mathrm{R}-\mathrm{M}$ curves. These two curves can be regarded as extensions of 


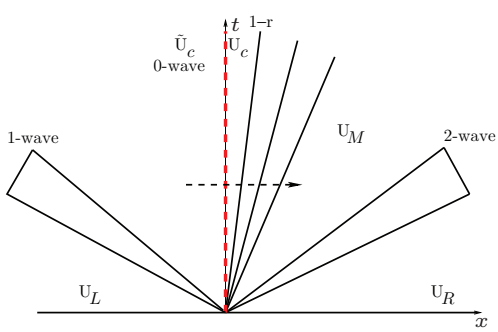

FIG. 6. $B$

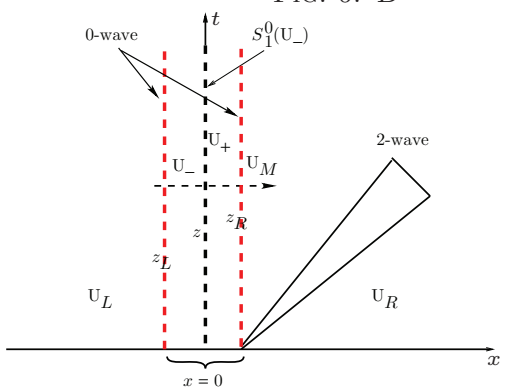

FIG. 8. $C$

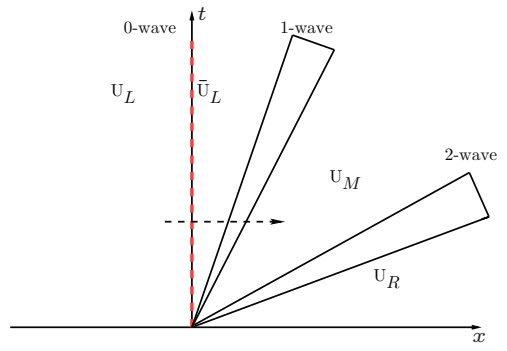

FIG. 10. D

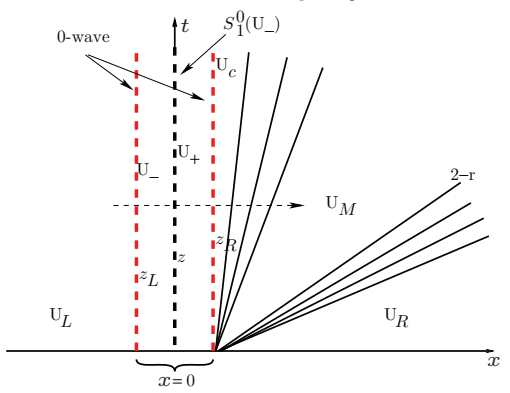

FIG. 12. $E$

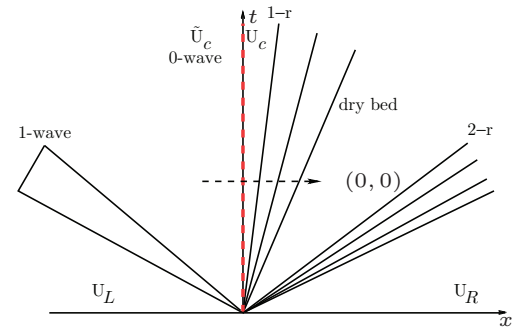

FIG. 7. $B_{v}$

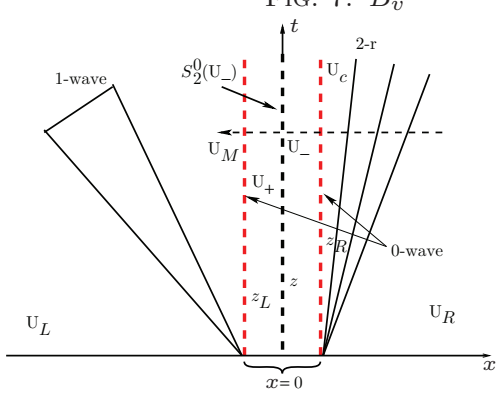

FIG. 9. F

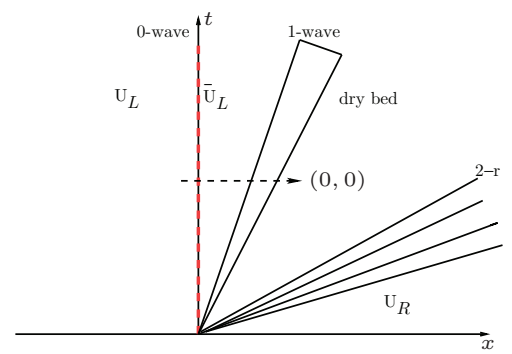

FIG. 11. $D_{v}$

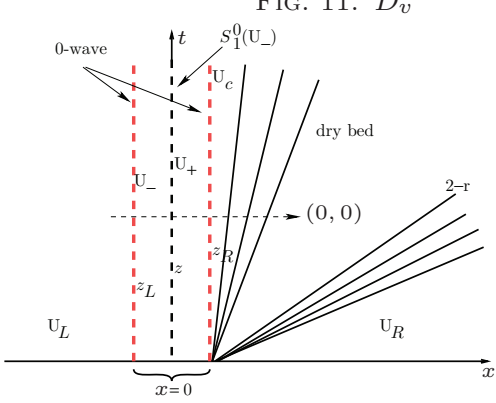

FIG. 13. $E_{v}$

the $T_{1}\left(\mathbf{U}_{L}\right)$ and $T_{2}\left(\mathbf{U}_{R}\right)$ curves respectively. They will serve as building blocks for the calculation of the Riemann solutions to the shallow water equation in a uniform way. 


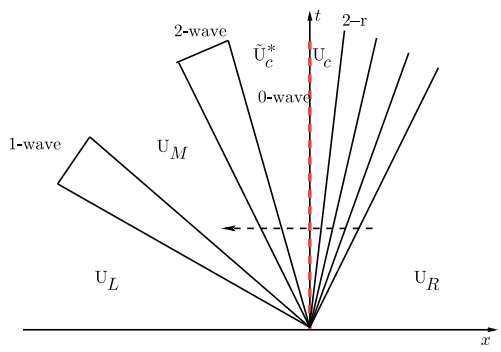

FIG. 14. $G$
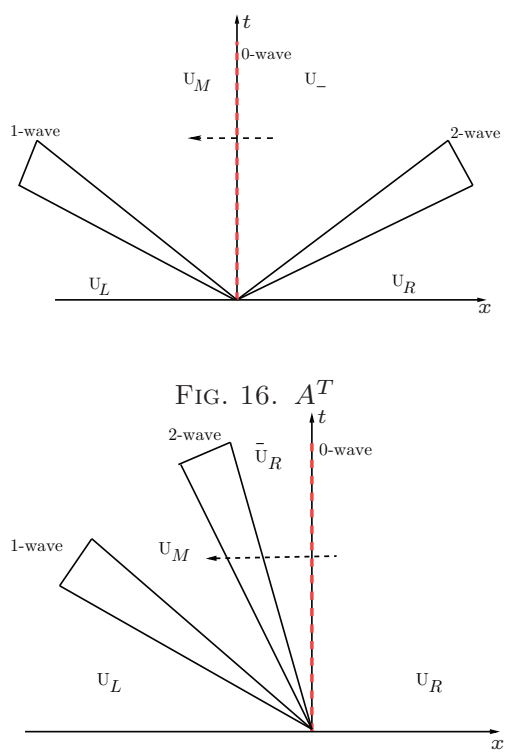

FiG. 18. $D^{T}$

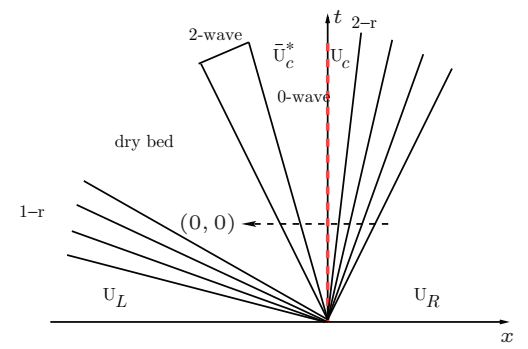

FIG. 15. $G_{v}$
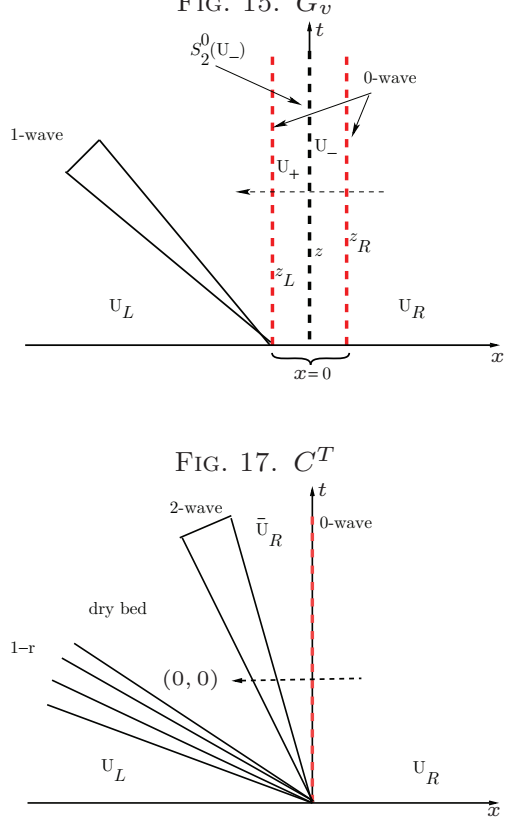

FiG. 19. $D_{v}^{T}$

There is precisely one stationary wave in a full wave curve from $\mathbf{U}_{L}$ to $\mathbf{U}_{R}$ located either on the $\mathrm{L}-\mathrm{M}$ curve or the $\mathrm{R}-\mathrm{M}$ curve. Due to the fact that the velocity does not change sign across the stationary wave, the location of the stationary wave is determined by this rule: If $u>0$ the stationary wave is on the $\mathrm{L}-\mathrm{M}$ curve; if $u<0$ the stationary wave is on the $\mathrm{R}-\mathrm{M}$ curve.

Hence if $u_{0 L}>0$ the $\mathrm{L}-\mathrm{M}$ curve always contains the segment

$$
P_{1}^{l}\left(\mathbf{U}_{L}\right)=\left\{\mathbf{U} \mid \mathbf{U} \in T_{1}\left(\mathbf{U}_{L}\right) \text { with } u \leq 0\right\} ;
$$

otherwise if $u_{0 L} \leq 0$ the $\mathrm{L}-\mathrm{M}$ will be

$$
P_{1}^{l}\left(\mathbf{U}_{L}\right)=\left\{\mathbf{U} \mid \mathbf{U} \in T_{1}\left(\mathbf{U}_{L}\right) \text { with } u \leq u_{0 L}\right\} .
$$

Similarly if $u_{0 R}<0$ the $\mathrm{R}-\mathrm{M}$ curve always contains the segment

$$
P_{1}^{r}\left(\mathbf{U}_{R}\right)=\left\{\mathbf{U} \mid \mathbf{U} \in T_{2}\left(\mathbf{U}_{R}\right) \text { with } u \geq 0\right\} ;
$$


otherwise if $u_{0 R} \geq 0$ the $\mathrm{R}-\mathrm{M}$ curve will be

$$
P_{1}^{r}\left(\mathbf{U}_{R}\right)=\left\{\mathbf{U} \mid \mathbf{U} \in T_{2}\left(\mathbf{U}_{R}\right) \text { with } u \geq u_{0 R}\right\} .
$$

It is necessary to construct the remaining segments of $\mathrm{L}-\mathrm{M}$ curves with $u_{0 L}>0$ and $u>0$, as well as for the $\mathrm{R}-\mathrm{M}$ curves with $u_{0 R}<0$ and $u<0$. Since $z_{L}<z_{R}$, Theorem 3.4 implies that the stationary wave always exists if the fluid flows from $z_{R}$ to $z_{L}$. However the stationary wave equations (3.4) and (3.5) may not have solutions if the fluid flows from $z_{L}$ to $z_{R}$. Before constructing the $\mathrm{L}-\mathrm{M}$ and $\mathrm{R}-\mathrm{M}$ curves, we need to consider the preliminaries for $\mathrm{L}-\mathrm{M}$ and $\mathrm{R}-\mathrm{M}$ curves first.

4.1. Preliminaries for $L-M$ curves with $u>0$. We now investigate the existence of the state $J\left(z_{R} ; \mathbf{U}_{-}, z_{L}\right)$, where $\mathbf{U}_{-} \in T_{1}\left(\mathbf{U}_{L}\right)$ and is connected to $\mathbf{U}_{L}$ by a negative-speed 1 -wave. Theorem 3.4 suggests the study of the following function

$$
\omega\left(h_{-}\right):=h_{-}\left(\frac{1}{2} F\left(h_{-}\right)^{2}-\frac{3}{2} F\left(h_{-}\right)^{\frac{2}{3}}+1\right)-\left(z_{R}-z_{L}\right),
$$

where the Froude number $F\left(h_{-}\right):=\frac{U\left(h_{-}\right)}{\sqrt{g h_{-}}}$and $U\left(h_{-}\right)=u_{L}-f\left(h_{-} ; h_{L}\right)$. Theorem 3.4 implies that if $\omega\left(h_{-}\right) \geq 0$ the state $J\left(z_{R} ; \mathbf{U}_{-}, z_{L}\right)$ exists and vice versa. So we need to study the behavior of $\omega\left(h_{-}\right)$.

Lemma 4.1. The function $\omega\left(h_{-}\right)$is strictly increasing if $0<F\left(h_{-}\right)<1$.

Proof. The function $\omega\left(h_{-}\right)$is continuous and differentiable. The derivative of $\omega\left(h_{-}\right)$ is

$$
\omega^{\prime}\left(h_{-}\right)=\frac{1}{2} F\left(h_{-}\right)^{2}-\frac{3}{2} F\left(h_{-}\right)^{\frac{2}{3}}+1+h_{-} F\left(h_{-}\right)^{-\frac{1}{3}}\left[F\left(h_{-}\right)^{\frac{4}{3}}-1\right] F^{\prime}\left(h_{-}\right),
$$

where by (2.19) and $U\left(h_{-}\right)>0$, we have

$$
F^{\prime}\left(h_{-}\right)=-\frac{f^{\prime}\left(h_{-} ; h_{L}\right)}{\sqrt{g h_{-}}}-\frac{U\left(h_{-}\right) \sqrt{g}}{2} h_{-}^{-\frac{3}{2}}<0 .
$$

As we have mentioned in (3.16),$\frac{1}{2} F\left(h_{-}\right)^{2}-\frac{3}{2} F\left(h_{-}\right)^{\frac{2}{3}}+1 \geq 0$. It takes the value 0 if and only if $F\left(h_{-}\right)=1$. So we obtain that $\omega^{\prime}\left(h_{-}\right)>0$ if $0<F\left(h_{-}\right)<1$ and $\omega^{\prime}\left(h_{-}\right)=0$ if $F\left(h_{-}\right)=1$.

Denote the minimum value of $h_{-}$as $h_{L}^{\min }$ and the maximum as $h_{L}^{\max }$. The curve $T_{1}\left(\mathbf{U}_{L}\right)$ is strictly decreasing in the $(u, h)$ state space. Also, $\mathbf{U}_{-} \in T_{1}\left(\mathbf{U}_{L}\right)$ is connected to $\mathbf{U}_{L}$ by a negative-speed 1-wave. Hence if $u_{L} \leq c_{L}, h_{L}^{\min }$ is the height corresponding to the sonic state on the curve $T_{1}\left(\mathbf{U}_{L}\right)$; while if $u_{L}>c_{L}, h_{L}^{\text {min }}$ is $\hat{h}_{L}$ which is defined in (2.12). That is to say we have

$$
h_{L}^{\min }= \begin{cases}\frac{\left(u_{L}+2 c_{L}\right)^{2}}{9 g}, & \text { if } u_{L} \leq c_{L}, \\ \hat{h}_{L}, & \text { if } u_{L}>c_{L} .\end{cases}
$$

Now we pay attention to $h_{L}^{\max }$. It should satisfy

$$
0=u_{L}-f\left(h_{-}^{\max } ; \mathbf{U}_{L}\right) .
$$

If $u_{L} \leq 0$, we have $h_{L}^{\max }<h_{L}$ which is the solution to the equation

$$
u_{L}-2\left(\sqrt{g h}-c_{L}\right)=0 .
$$


This leads to $h_{L}^{\max }=\frac{\left(u_{L}+2 c_{L}\right)^{2}}{4 g}$. Otherwise if $u_{L}>0$, we have $h_{L}^{\max }>h_{L}$. Hence from (2.16) it is the solution of the equation

$$
u_{L}-\left(h-h_{L}\right) \sqrt{\frac{g}{2}\left(\frac{1}{h}+\frac{1}{h_{L}}\right)}=0 .
$$

After a short calculation we have

$$
\left(\frac{h}{h_{L}}\right)^{3}-\left(\frac{h}{h_{L}}\right)^{2}-\left(1+2 F_{L}^{2}\right) \frac{h}{h_{L}}+1=0 .
$$

Setting $x=\frac{h}{h_{L}}>1$, (4.11) becomes

$$
f(x)=x^{3}-x^{2}-\left(1+2 F_{L}^{2}\right) x+1 .
$$

Direct calculation yields the following facts. The function $f(x)$ defined in (4.12) reaches the maximum at $x_{l}:=\frac{1}{3}-\frac{2}{3} \sqrt{1+\frac{3}{2} F_{L}^{2}}<0$ and the minimum at $x_{r}:=\frac{1}{3}+\frac{2}{3} \sqrt{1+\frac{3}{2} F_{L}^{2}}>$ 1. When $x<x_{l}, f(x)$ increases from $-\infty$ to the maximum value at $x=x_{l}$. When $x \in] x_{l}, x_{r}$, it decreases from the maximum value to the minimum value at $x=x_{r}$. When $x>x_{r}$, it increases from the minimum value to $\infty$. Furthermore, be advised that $x_{l}<1<x_{r}$ and $f(1)=-2 F_{L}^{2}<0$, so $f\left(x_{r}\right)<f(1)<0$. Thus there is exactly one real solution to the cubic equation $f(x)=0$ when $x>x_{r}>1$. We denote this solution as $x_{u_{1}^{0}}$ which can be directly calculated by the method for the exact solution to cubic equations; see Nickalls [8]. Finally we have

$$
h_{L}^{\max }= \begin{cases}\frac{\left(u_{L}+2 c_{L}\right)^{2}}{4 g}, & \text { if } u_{L} \leq 0, \\ h_{L} x_{u_{l}^{0}}, & \text { if } u_{L}>0 .\end{cases}
$$

Thus the reasonable region for $\omega\left(h_{-}\right)$is $] h_{L}^{\min }, h_{L}^{\max }$ [. Moreover, we have the following lemma.

Lemma 4.2. Set

$$
z_{\max }:=z_{L}+h_{L}^{\max }
$$

The stationary state $\mathbf{U}=J\left(z_{R} ; \mathbf{U}_{-}, z_{L}\right)$ with $0<u_{-} \leq c_{-}$cannot exist if $z_{\max }<z_{R}$.

Proof. Note that $\omega\left(h_{L}^{\max }\right)=h_{L}^{\max }-\left(z_{R}-z_{L}\right)=z_{\max }-z_{R}$. So if $z_{\max }<z_{R}$, $\omega\left(h_{L}^{\max }\right)<0$. The function $\omega\left(h_{-}\right)$is increasing in terms of $\left.h_{-} \in\right] h_{L}^{\min }, h_{L}^{\max }$ [. Hence $\omega\left(h_{L}^{\min }\right)<\omega\left(h_{-}\right) \leq \omega\left(h_{L}^{\max }\right)<0$ if $z_{\max }<z_{R}$. Theorem 3.4 implies that if $\omega\left(h_{-}\right)<0$, the stationary wave $\mathbf{U}=J\left(z_{R} ; \mathbf{U}_{-}, z_{L}\right)$ cannot exist.

Lemma 4.3. Suppose that $z_{R}<z_{\max }$ and $u_{L}<c_{L}$. There exists a state $\tilde{\mathbf{U}}_{c} \in T_{1}\left(\mathbf{U}_{L}\right)$ which satisfies $\mathbf{U}_{c}=J\left(z_{R} ; \tilde{\mathbf{U}}_{c}, z_{L}\right)$.

Proof. Due to $z_{R}<z_{\max }$, we have $\omega\left(h_{L}^{\max }\right)=z_{\max }-z_{L}>0$ and $h_{L}^{\min }=\frac{\left(u_{L}+2 c_{L}\right)^{2}}{9 g}$. A short calculation yields that $\omega\left(h_{L}^{\text {min }}\right)=z_{L}-z_{R}<0$. The function $\omega\left(h_{-}\right)$is continuous and increasing. By the intermediate value theorem there is a unique solution to $\omega\left(h_{-}\right)=$ 0 . Denote the solution to $\omega\left(h_{-}\right)=0$ as $\tilde{h}_{c}$. Then the corresponding velocity $\tilde{u}_{c}$ can be calculated by

$$
\tilde{u}_{c}=u_{L}-f\left(\tilde{h}_{c} ; \mathbf{U}_{L}\right)
$$


The velocity function of $J\left(z_{R} ; \tilde{\mathbf{U}}_{c}, z_{L}\right)$ is

$$
\Psi\left(u ; \tilde{\mathbf{U}}_{c}, z_{L}, z_{R}\right):=\frac{u^{2}}{2}+\frac{\tilde{c}_{c}^{2} \tilde{u}_{c}}{u}-\frac{\tilde{u}_{c}^{2}}{2}-g \tilde{h}_{c}+g\left(z_{R}-z_{L}\right) .
$$

The minimum of this velocity function is

$$
\Psi\left(u^{*} ; \tilde{\mathbf{U}}_{c}, z_{L}, z_{R}\right)=g \omega\left(\tilde{h}_{c}\right)=0 .
$$

Hence Remark 3.3 implies that the outflow state of stationary wave is a sonic state, i.e. $\mathbf{U}_{c}=J\left(z_{R} ; \tilde{\mathbf{U}}_{c}, z_{L}\right)$.

REMARK 4.4. Lemma 4.3 is totally consistent with Lemma 3.1

Note that Lemma 4.2 states that in this case the flow coming from the left cannot spill over the obstacle caused by the jump in the bed height at $x=0$, whereas in the case of Lemma 4.3, overspill occurs if the velocity is large enough, leading to $\omega\left(h_{-}\right)>0$.

In the case when $z_{R}<z_{\max }$ and $u_{L}>c_{L}$, we have $h_{L}^{\text {min }}=\hat{h}_{L}$. We define two critical bottom steps

$$
z_{S}=z_{L}+\hat{h}_{L}\left(\frac{1}{2} \hat{F}_{L}^{2}-\frac{3}{2} \hat{F}_{L}^{\frac{2}{3}}+1\right)
$$

and

$$
z_{T}=z_{L}+h_{L}\left(\frac{1}{2} F_{L}^{2}-\frac{3}{2} F_{L}^{\frac{2}{3}}+1\right)
$$

where $\hat{h}_{L}$ and $\hat{u}_{L}$ were defined in (2.12) and (2.13) respectively. The Froude number

$$
\hat{F}_{L}=\frac{\hat{u}_{L}}{\hat{c}_{L}} .
$$

Since $\hat{c}_{L}=\sqrt{g \hat{h}_{L}}$, taking (2.12) and (2.13) into (4.18), we obtain

$$
\hat{F}_{L}=\frac{1}{8} F_{L}^{-2}\left[1+\sqrt{1+8 F_{L}^{2}}\right]^{\frac{3}{2}} .
$$

We invoke the existence condition for resonant waves due to the coincidence of a 0 -speed shock and the stationary wave.

Lemma 4.5. Suppose $z_{L}<z_{R}<z_{\max }$ and $u_{L}>c_{L}$. We have the following facts.

(1) The state $\mathbf{U}=J\left(z_{R} ; S_{1}^{0}\left(\mathbf{U}_{L}\right), z_{L}\right)$ exists if $z_{R} \leq z_{S}$; otherwise it fails to exist.

(2) The state $\mathbf{U}=J\left(z_{R} ; \mathbf{U}_{L}, z_{L}\right)$ exists if $z_{R} \leq z_{T}$; otherwise it fails to exist.

(3) One always has $z_{T}>z_{S}$.

Proof. From Theorem 3.4 the existence condition for the state $\mathbf{U}=J\left(z_{R} ; S_{1}^{0}\left(\mathbf{U}_{L}\right), z_{L}\right)$ is that

$$
z_{R}<z_{L}+\hat{h}_{L}\left(\frac{1}{2}\left(\hat{F}_{L}\right)^{2}-\frac{3}{2}\left(\hat{F}_{L}\right)^{\frac{2}{3}}+1\right)=z_{S} .
$$

Analogously we can prove the second statement. Now we investigate the relationship between $z_{S}$ and $z_{T}$. From (2.12) and (4.19), we have

$$
z_{S}=z_{L}+h_{L}\left[\frac{1}{32 F_{L}^{2}}\left(1+\sqrt{1+8 F_{L}^{2}}\right)^{2}+\frac{-1+\sqrt{1+8 F_{L}^{2}}}{2}-\frac{3}{2} F_{L}^{\frac{2}{3}}\right] .
$$


By (4.16) and (4.21), we have

$$
\begin{aligned}
z_{T}-z_{S} & =h_{L}\left[\frac{1}{2} F_{L}^{2}-\frac{3}{2} F_{L}^{\frac{2}{3}}+1-\frac{1}{32 F_{L}^{2}}\left(1+\sqrt{1+8 F_{L}^{2}}\right)^{2}-\frac{-1+\sqrt{1+8 F_{L}^{2}}}{2}+\frac{3}{2} F_{L}^{\frac{2}{3}}\right], \\
& =\frac{h_{L}}{F_{L}^{2}}\left[\frac{1}{2} F_{L}^{4}+F_{L}^{2}-\frac{1}{16}\left(1+4 F_{L}^{2}+\sqrt{1+8 F_{L}^{2}}\right)-\frac{-1+\sqrt{1+8 F_{L}^{2}}}{2} F_{L}^{2}\right], \\
& =\frac{h_{L}}{F_{L}^{2}}\left[\frac{1}{2} F_{L}^{4}+\frac{5}{4} F_{L}^{2}-\frac{1}{16}-\frac{\left(1+8 F_{L}^{2}\right)^{\frac{3}{2}}}{16}\right], \\
& =\frac{h_{L}}{128 F_{L}^{2}}\left[-3+\sqrt{1+8 F_{L}^{2}}\right]^{3}\left[1+\sqrt{1+8 F_{L}^{2}}\right] . \\
& >0
\end{aligned}
$$

when $F_{L}^{2}>1$.

Assume that $z_{L}<z_{R}$ and $u_{L}>c_{L}$. We now consider resonant waves due to the coincidence of the 0 -speed 1 -shock with stationary waves. The 0 -speed 1 -shock splits the stationary wave into a supersonic part and a subsonic part. The corresponding wave curve is defined as follows.

$$
\left\{\mathbf{U} \mid \mathbf{U}=\mathbf{J}\left(z_{R} ; \mathbf{U}_{+}, z\right) ; \mathbf{U}_{+}=S_{1}^{0}\left(\mathbf{U}_{-}\right) ; \mathbf{U}_{-}=\mathbf{J}\left(z ; \mathbf{U}_{L}, z_{L}\right)\right\},
$$

where $z \in] z_{L}, z_{R}$. We denote the Froude numbers for the states $\mathbf{U}_{ \pm}$in (4.22) as $F_{ \pm}=$ $\frac{u_{ \pm}}{\sqrt{g h_{ \pm}}}$. By using (3.4) we have

$$
h_{+} u_{+}=h_{-} u_{-}=h_{L} u_{L}
$$

Therefore we obtain the functions $F_{ \pm}$in terms of $h_{ \pm}$respectively:

$$
F\left(h_{ \pm}\right):=F_{ \pm}=\frac{u_{L}^{2} h_{L}^{2}}{\sqrt{g} h_{ \pm}^{\frac{3}{2}}} .
$$

By (4.23), the derivatives of the functions $F\left(h_{ \pm}\right)$are

$$
\frac{d F\left(h_{ \pm}\right)}{d h_{ \pm}}=-\frac{3}{2} \frac{F_{ \pm}}{h_{ \pm}}
$$

Similar to (4.19), we obtain the further relations for $F_{-}$and $F_{+}$

$$
F_{+}=\frac{1}{8} F_{-}^{-2}\left(1+\sqrt{1+8 F_{-}^{2}}\right)^{\frac{3}{2}} .
$$

The resonant wave curve in (4.22) is viewed as a function of $z$. Actually the variable $h_{-}$ is more convenient to analyze the existence of the wave curve in (4.22). Specifically, the following lemma holds.

Lemma 4.6. For the supersonic state $\mathbf{U}_{-}=\mathbf{J}\left(z ; \mathbf{U}_{L}, z_{L}\right)$ in (4.22) with $z_{L} \leq z \leq z_{R}$, we have

$$
h_{L} \leq h_{-} \leq \bar{h}_{L}
$$

where $\overline{\mathbf{U}}_{L}=\mathbf{J}\left(z_{R} ; \mathbf{U}_{L}, z_{L}\right)$. 
Proof. Considering (3.4) and (3.5) for $\mathbf{U}_{-}=\mathbf{J}\left(z ; \mathbf{U}_{L}, z_{L}\right)$, we study the following equation:

$$
\frac{h_{L}^{2} u_{L}^{2}}{2 g h_{-}^{2}}+h_{-}+z-\frac{u_{L}^{2}}{2 g}-h_{L}-z_{L}=0 .
$$

Taking $z$ as a function of $h_{-}$, we obtain

$$
z\left(h_{-}\right):=-\frac{h_{L}^{2} u_{L}^{2}}{2 g h_{-}^{2}}-h_{-}+\frac{u_{L}^{2}}{2 g}+h_{L}+z_{L} .
$$

Using (4.23), we have

$$
\frac{d z\left(h_{-}\right)}{d h_{-}}=F_{-}^{2}-1>0
$$

Note that $h_{-}=h_{L}$ when $z=z_{L}$, while $h_{-}=\bar{h}_{L}$ when $z=z_{R}$. Thus (4.30) implies that $h_{L} \leq h_{-} \leq \bar{h}_{L}$.

To prove the existence of the wave curve defined in (4.22), we have to study the existence of the supersonic state $\mathbf{U}_{-}=\mathbf{J}\left(z ; \mathbf{U}_{L}, z_{L}\right)$ and the subsonic state $\mathbf{U}=\mathbf{J}\left(z_{R} ; \mathbf{U}_{+}, z\right)$ with $z_{L} \leq z \leq z_{R}$. We present the details in the following lemmas.

Lemma 4.7. The region of $z$ for the existence of the subsonic state $\mathbf{U}=\mathbf{J}\left(z_{R} ; \mathbf{U}_{+}, z\right)$ defined in (4.22) is as follows:

(1) $z \in] z_{L}, z_{R}\left[\right.$ if $z_{S} \geq z_{R}$;

(2) $z \in] z_{c}, z_{R}$ if $z_{S}<z_{R}$ where $z_{c}$ is defined in (4.43).

Proof. Theorem 3.4 implies that $\mathbf{U}=\mathbf{J}\left(z_{R} ; \mathbf{U}_{+}, z\right)$ exists if

$$
z_{R}-z \leq h_{+}\left(\frac{1}{2} F_{+}^{2}-\frac{3}{2} F_{+}^{\frac{2}{3}}+1\right) .
$$

In addition, by (2.12) and (2.13), we have

$$
h_{+}=\frac{h_{-}}{2}\left(-1+\sqrt{1+8 F_{-}^{2}}\right) .
$$

That is to say, $h_{+}$can be treated as a function of $h_{-}$. This suggests to consider the function

$$
\Theta\left(h_{-}\right):=h_{+}\left(\frac{1}{2} F\left(h_{+}\right)^{2}-\frac{3}{2} F\left(h_{+}\right)^{\frac{2}{3}}+1\right)+z-z_{R} .
$$

For simplicity we introduce the function

$$
A\left(h_{+}\right):=h_{+}\left(\frac{1}{2} F\left(h_{+}\right)^{2}-\frac{3}{2} F\left(h_{+}\right)^{\frac{2}{3}}+1\right) .
$$

Therefore $\Theta\left(h_{-}\right)$in (4.33) can be rewritten as

$$
\Theta\left(h_{-}\right)=A\left(h_{+}\left(h_{-}\right)\right)+z\left(h_{-}\right)-z_{R} .
$$

By the chain rule we have

$$
\Theta^{\prime}\left(h_{-}\right)=\frac{d A\left(h_{+}\right)}{d h_{+}} \frac{d h_{+}}{d h_{-}}+\frac{d z\left(h_{-}\right)}{d h_{-}} .
$$

Using (4.25) we obtain

$$
\frac{d h_{+}}{d h_{-}}=-\frac{1}{2}+\frac{1-4 F_{-}^{2}}{2 \sqrt{1+8 F_{-}^{2}}}
$$


Besides, by (4.25) and (4.26), we have

$$
\begin{aligned}
\frac{d A\left(h_{+}\right)}{d h_{+}} & =\frac{1}{2} F\left(h_{+}\right)^{2}-\frac{3}{2} F\left(h_{+}\right)^{\frac{2}{3}}+1+h_{+}\left(F\left(h_{+}\right)-F\left(h_{+}\right)^{-\frac{1}{3}}\right) \frac{d F\left(h_{+}\right)}{d h_{+}} \\
& =\frac{1}{2} F\left(h_{+}\right)^{2}-\frac{3}{2} F\left(h_{+}\right)^{\frac{2}{3}}+1-\frac{3}{2} F\left(h_{+}\right)\left(F\left(h_{+}\right)-F\left(h_{+}\right)^{-\frac{1}{3}}\right) \\
& =1-F\left(h_{+}\right) \\
& =1-\frac{1}{8} F_{-}^{-2}\left(1+\sqrt{1+8 F_{-}^{2}}\right)^{\frac{3}{2}} .
\end{aligned}
$$

By (4.30), (4.36) as well as (4.37), we have

$$
\begin{aligned}
\Theta^{\prime}\left(h_{-}\right) & =\left(1-\frac{1}{8} F_{-}^{-2}\left(1+\sqrt{1+8 F_{-}^{2}}\right)^{\frac{3}{2}}\right)\left(-\frac{1}{2}+\frac{1-4 F_{-}^{2}}{2 \sqrt{1+8 F_{-}^{2}}}\right)+F_{-}^{2}-1 \\
& =\frac{\left(3+\sqrt{1+8 F_{-}^{2}}\right)\left[\left(-\frac{5}{2}+\sqrt{1+8 F_{-}^{2}}\right)^{2}+2\left(1+\sqrt{1+8 F_{-}^{2}}\right)^{\frac{1}{2}}-\frac{17}{4}\right]}{8 \sqrt{1+8 F_{-}^{2}}} . \\
& >0
\end{aligned}
$$

when $F_{-}^{2}>1$. By Lemma 4.27, we have $h_{L} \leq h_{-} \leq \bar{h}_{L}$. Thus, due to (4.38), we have

$$
\Theta\left(h_{L}\right) \leq \Theta\left(h_{-}\right) \leq \Theta\left(\bar{h}_{L}\right) .
$$

From (4.31), the state $\mathbf{J}\left(z_{R} ; \mathbf{U}_{+}, z\right)$ exists if $\Theta\left(h_{-}\right) \geq 0$. Remember that we denote $\hat{\overline{\mathbf{U}}}_{L}=S_{k}^{0}\left(\overline{\mathbf{U}}_{L}\right)$. We have

$$
\Theta\left(\bar{h}_{L}\right)=\hat{\bar{h}}_{L}\left(\frac{1}{2} \hat{\bar{F}}_{L}^{2}-\frac{3}{2} \hat{\bar{F}}_{L}^{\frac{2}{3}}+1\right) \geq 0
$$

From (4.16) as well as (4.33), we obtain that

$$
\Theta\left(h_{L}\right)=z_{S}-z_{R}
$$

So, on one hand, if $z_{S} \geq z_{R}$, we have $0 \leq \Theta\left(h_{L}\right) \leq \Theta\left(h_{-}\right) \leq \Theta\left(\bar{h}_{L}\right)$. Thus the state $\mathbf{J}\left(z_{R} ; \mathbf{U}_{+}, z\right)$ exists for any $z_{L} \leq z \leq z_{R}$. On the other hand, if $z_{S}<z_{R}$, we have $\Theta\left(h_{L}\right)<0<\Theta\left(\bar{h}_{L}\right)$. From the intermediate value theorem there is a unique solution, denoted as $\tilde{h}_{c_{s}}$, to the equation $\Theta\left(h_{-}\right)=0$ where $\left.h_{-} \in\right] h_{L}, \bar{h}_{L}[$. The corresponding velocity can be calculated from

$$
\tilde{u}_{c_{s}}=\frac{h_{L} u_{L}}{\tilde{h}_{c_{s}}},
$$

and the related bottom step denoted as $z_{c}$ can be deduced from equation (4.28), i.e.

$$
z_{c}=-\frac{h_{L}^{2} u_{L}^{2}}{2 g \tilde{h}_{c_{s}}^{2}}+\tilde{h}_{c_{s}}-\frac{u_{L}^{2}}{2 g}-h_{L}-z_{L} .
$$

Hence $\Theta\left(h_{-}\right) \geq 0$ if $z_{c} \leq z \leq z_{R}$.

Lemma 4.8. Assume that $u_{L}>c_{L}$ for $z_{T}$, given by (4.17); we have $z_{T}<z_{c}$ if $z_{T}<z_{R}$. 
Proof. Denote $\mathbf{U}_{c, l}^{*}=\mathbf{J}\left(z_{R} ; \mathbf{U}_{+}, z_{T}\right)$. Taking $z=z_{T}$ in (4.28), we obtain that

$$
\alpha\left(h_{-}\right):=\frac{h_{L}^{2} u_{L}^{2}}{2 h_{-}^{2}}+g h_{-}-\frac{3}{2}\left(u_{L} c_{L}^{2}\right)^{\frac{2}{3}}=0 .
$$

The function $\alpha\left(h_{-}\right)$is continuous and differentiable. The derivative of this function is

$$
\alpha^{\prime}\left(h_{-}\right)=-\frac{h_{L}^{2} u_{L}^{2}}{h_{-}^{3}}+g
$$

Setting $h^{*}=h_{L} F_{L}^{\frac{2}{3}}$, we have $\alpha^{\prime}\left(h_{-}\right)<0$ if $h_{-}<h^{*}$, while $\alpha^{\prime}\left(h_{-}\right)>0$ if $h_{-}>h^{*}$. It has the minimum value at $h_{-}=h^{*}$ and $\alpha\left(h^{*}\right)=0$. Therefore there is a unique solution to $\alpha\left(h_{-}\right)=0$, i.e. $h_{c, l}^{*}=h^{*}=h_{L} F_{L}^{\frac{2}{3}}$. Using (4.23) we obtain that

$$
u_{c, l}^{*}=u_{L} F_{L}^{-\frac{2}{3}}=c_{L} F_{L}^{\frac{1}{3}}=\sqrt{g h_{c, l}^{*}}=c_{c, l}^{*} .
$$

Thus the state $\mathbf{U}_{c, l}^{*}$ is the sonic state. Hence we have $h^{+}=h_{c, l}^{*}$ and $F^{+}=1$ in (4.32) and (4.24) respectively. From (4.6) we have $\Theta\left(h_{c, l}^{*}\right)=z_{T}-z_{R}<0$ if $z_{T}<z_{R}$. Since $\Theta\left(\tilde{h}_{c_{s}}\right)=0$, we have by (4.38) $h_{c, l}^{*}<\tilde{h}_{c_{s}}$. Consequently we have $z_{T}<z_{c}$ due to (4.29).

Based on Lemmas 4.6, 4.7, and 4.8, we now study the existence region for the wave curve defined in (4.22).

Lemma 4.9. Assume that $z_{L}<z_{R}$ and $u_{L}>c_{L}$; then we have

(1) if $z_{R} \leq z_{S}<z_{T}$, the curve in (4.22) exists.

(2) if $z_{S}<z_{R} \leq z_{T}$, the curve in (4.22) exists when $\left.z \in\right] z_{c}, z_{R}[$.

(3) if $z_{S}<z_{T}<z_{R}$, the curve in (4.22) fails to exist.

Proof. The wave curve defined in (4.22) exists if the two states $\mathbf{U}_{-}=\mathbf{J}\left(z ; \mathbf{U}_{L}, z_{L}\right)$ and $\mathbf{U}=\mathbf{J}\left(z_{R} ; \mathbf{U}_{+}, z\right)$ exist. Lemma 4.5 implies that the state $\mathbf{U}_{-}=\mathbf{J}\left(z ; \mathbf{U}_{L}, z_{L}\right)$ exists if $z \leq z_{T}$.

Thus in one case when $z_{R}<z_{T}$, the state $\mathbf{U}_{-}$defined in (4.22) with $\left.z \in\right] z_{L}, z_{R}$ [ always exists. Lemma 4.7 conveys that on one hand if $z_{S} \geq z_{R}$ the state $\mathbf{U}=\mathbf{J}\left(z_{R} ; \mathbf{U}_{+}, z\right)$ exists when $z \in] z_{L}, z_{R}\left[\right.$. Thus the first statement is true due to $z_{S}<z_{T}$ by Lemma 4.5. On the other hand, if $z_{S}<z_{R}$, the state $\mathbf{U}=\mathbf{J}\left(z_{R} ; \mathbf{U}_{+}, z\right)$ exists when $\left.z \in\right] z_{c}, z_{R}[$. This is the second statement.

In the other case when $z_{R}>z_{T}$, the state $\mathbf{U}_{-}$exists if $\left.z \in\right] z_{L}, z_{T}[$. By Lemmas 4.8 and 4.7 we have $] z_{L}, z_{T}[\cap] z_{c}, z_{R}[=\emptyset$. This is sufficient for the third statement.

REMARK 4.10. Suppose that we have $z_{L}<z_{R}, u_{L}>c_{L}$ and $z_{S}<z_{R}<z_{T}$. Lemma 4.9 reveals that there exists an $\tilde{h}_{c_{s}}$ such that $\Theta\left(\tilde{h}_{c_{s}}\right)=0$. Moreover, note that $\Theta\left(\tilde{h}_{c_{s}}\right)$ is the minimum value of the velocity function to $\mathbf{J}\left(z_{R} ; \hat{\mathbf{U}}_{c_{s}}, z_{c}\right)$; i.e. the outflow state of $\mathbf{J}\left(z_{R} ; \hat{\mathbf{U}}_{c_{s}}, z_{c}\right)$ is the sonic state. We denote it as $\mathbf{U}_{c_{3}}$, i.e. $\mathbf{U}_{c_{3}}=\mathbf{J}\left(z_{R} ; \hat{\mathbf{U}}_{c_{s}}, z_{c}\right)$.

REMARK 4.11. Suppose $z_{S}<z_{R}<z_{T}$ and $u_{L}>c_{L}$, i.e. $h_{L}^{\text {min }}=\hat{h}_{L}$. Note that

$$
\omega\left(\hat{h}_{L}\right)=z_{S}-z_{R}<0 .
$$

Analogously to Lemma 4.3, there is a unique solution to $\omega\left(h_{-}\right)=0$. Here we denote this as $\tilde{h}_{c}^{L}$. The corresponding velocity $\tilde{u}_{c}^{L}$ can be calculated from (4.15) by setting $\tilde{h}_{c}=\tilde{h}_{c}^{L}$. 
Also we have $\mathbf{U}_{c_{2}}=J\left(z_{R} ; \tilde{\mathbf{U}}_{c}, z_{L}\right)$, where $\mathbf{U}_{c_{2}}$ is the sonic state. The subscript 2 is used to distinguish the sonic state $\mathbf{U}_{c_{2}}$ from the sonic state $\mathbf{U}_{c_{3}}$ in Remark 4.10.

4.1.1. Monotonicity. In this section we consider the monotonicity of two types of curves as the preliminary step for the study of the $\mathrm{L}-\mathrm{M}$ and $\mathrm{R}-\mathrm{M}$ curves.

We define

$$
P^{l}\left(\mathbf{U}_{R}\right)=\left\{\mathbf{U} \mid \mathbf{U}=\mathbf{J}\left(z_{R} ; \mathbf{U}_{-}, z_{L}\right) \text { and } \mathbf{U}_{-} \in T_{1}\left(\mathbf{U}_{L}\right)\right\}
$$

where $h_{L}^{\min }<h_{-}<h_{L}^{\max }$ and

$$
P^{r}\left(\mathbf{U}_{R}\right)=\left\{\mathbf{U} \mid \mathbf{U}=\mathbf{J}\left(z_{L} ; \mathbf{U}_{-}, z_{R}\right) \text { and } \mathbf{U}_{-} \in T_{2}\left(\mathbf{U}_{R}\right)\right\}
$$

where $0<u_{-}+c_{-}<c_{-}$. Note that $P^{l}\left(\mathbf{U}_{L}\right)$ and $P^{r}\left(\mathbf{U}_{R}\right)$ are the composites of the 1or 2-wave curve with a stationary wave. Before studying the behavior of $P^{l}\left(\mathbf{U}_{L}\right)$ and $P^{r}\left(\mathbf{U}_{R}\right)$, we consider the following lemma first.

Lemma 4.12. For any state $\mathbf{U}_{-} \in T_{1}\left(\mathbf{U}_{L}\right)$ connected to $\mathbf{U}_{L}$ by a negative speed 1-wave, we have

$$
u_{-}-h_{-} f^{\prime}\left(h_{-} ; h_{L}\right)<0, \quad \text { and } \quad u_{-} f^{\prime}\left(h_{-} ; h_{L}\right)-g<0 .
$$

Proof. We have $u_{-}-c_{-}<0$ since the states $\mathbf{U}_{-}$and $\mathbf{U}_{L}$ are connected by a negative speed 1-wave. From (2.18) we have

$$
u_{-}-h_{-} f^{\prime}\left(h_{-} ; h_{L}\right)= \begin{cases}u_{-}-c_{-}, & \text {if } h_{-} \leq h_{L}, \\ u_{-}-h_{-} \sqrt{\frac{g}{2}} \frac{\frac{1}{h_{-}}+\frac{2}{h_{L}}+\frac{h_{L}}{h_{-}^{2}}}{2 \sqrt{\frac{1}{h_{-}}+\frac{1}{h_{L}}}}, & \text { if } h_{-}>h_{L} .\end{cases}
$$

If $h_{-} \leq h_{L}$, obviously we have $u_{-}-h_{-} f^{\prime}\left(h_{-} ; h_{L}\right)<0$. Otherwise if $h_{-}>h_{L}$, we have

$$
\begin{aligned}
\sqrt{\frac{g}{2} \frac{\frac{1}{h_{-}}+\frac{2}{h_{L}}+\frac{h_{L}}{h_{-}^{2}}}{2 \sqrt{\frac{1}{h_{-}}+\frac{1}{h_{L}}}}} & =\frac{\sqrt{g}}{2 \sqrt{2}}\left(\sqrt{\frac{1}{h_{-}}+\frac{1}{h_{L}}}+\frac{\frac{1}{h_{L}}+\frac{h_{L}}{h_{-}^{2}}}{\sqrt{\frac{1}{h_{-}}+\frac{1}{h_{L}}}}\right) \\
& \geq \sqrt{\frac{g}{2}} \sqrt{\frac{1}{h_{L}}+\frac{h_{L}}{h_{-}^{2}}}>\sqrt{\frac{g}{h_{-}}}
\end{aligned}
$$

So using (4.51) in 4.50), we obtain

$$
u_{-}-h_{-} f^{\prime}\left(h_{-} ; h_{L}\right)<u_{-}-c_{-} .
$$

Hence we have $u_{-}-h_{-} f^{\prime}\left(h_{-} ; h_{L}\right)<0$ due to $u_{-}-c_{-}<0$.

Now we turn to $u_{-} f^{\prime}\left(h_{-} ; h_{L}\right)-g$. Note that

$$
u_{-} f^{\prime}\left(h_{-} ; h_{L}\right)-g= \begin{cases}\sqrt{\frac{g}{h_{-}}}\left(u_{-}-c_{-}\right)<0, & \text { if } h_{-} \leq h_{L}, \\ u_{-} \sqrt{\frac{g}{2}} \frac{\frac{1}{h_{-}}+\frac{2}{h_{L}}+\frac{h_{L}}{h_{-}^{2}}}{2 \sqrt{\frac{1}{h_{-}}+\frac{1}{h_{L}}}}-g, & \text { if } h_{-}>h_{L} .\end{cases}
$$

So it is only necessary to consider the case that $h_{-}>h_{L}$. To ensure that the $1-$ wave has a negative speed, we have $h_{-}>h_{-}^{\text {min }}$ where

$$
h_{-}^{\text {min }}= \begin{cases}h_{L}, & \text { if } u_{L} \leq c_{L}, \\ \hat{h}_{L}, & \text { if } u_{L}>c_{L},\end{cases}
$$


where $\hat{h}_{L}$ was defined in (2.12). Besides, by (2.20), we have

$$
\frac{\partial u_{-} f_{L}^{\prime}\left(h_{-} ; h_{L}\right)}{\partial h_{-}}=-\left(f_{L}^{\prime}\left(h_{-} ; h_{L}\right)\right)^{2}+u_{-} f_{L}^{\prime \prime}\left(h_{-} ; h_{L}\right)<0 .
$$

Therefore, when $h_{-}>h_{L}$, we have

$$
u_{-} f_{L}^{\prime}\left(h_{-} ; h_{L}\right)<u_{-}^{m i n} f_{L}^{\prime}\left(h_{-}^{m i n} ; \mathbf{U}_{L}\right),
$$

where $u_{-}^{\text {min }}=u_{L}-f\left(h_{-}^{\text {min }} ; \mathbf{U}_{L}\right)$. Specifically, by (4.54), we have

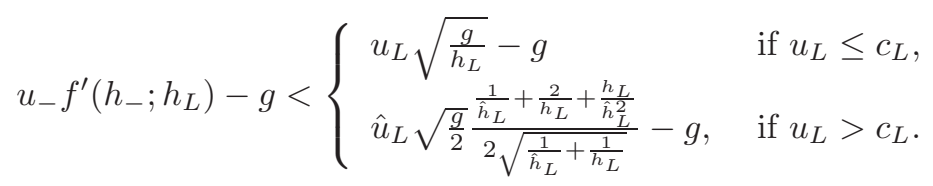

Note that when $u_{L} \leq c_{L}, u_{-} f^{\prime}\left(h_{-} ; h_{L}\right)-g<\sqrt{\frac{g}{h_{L}}}\left(u_{L}-c_{L}\right) \leq 0$. Now we consider the case when $u_{L}>c_{L}$. By $\hat{u}_{L}=\frac{h_{L} u_{L}}{\hat{h}_{L}}$, we have

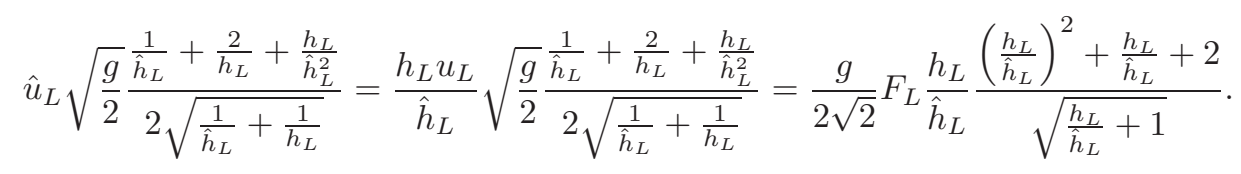

Moreover, from (2.12), we obtain that

$$
\frac{h_{L}}{\hat{h}_{L}}=\frac{1+\sqrt{1+8 F_{L}^{2}}}{4 F_{L}^{2}} .
$$

Set $x=\frac{h_{L}}{\hat{h}_{L}}$. Then $F_{L}=\frac{\sqrt{x+1}}{\sqrt{2} x}$. So we have

$$
\frac{g}{2 \sqrt{2}} F_{L} \frac{h_{L}}{\hat{h}_{L}} \frac{\left(\frac{h_{L}}{\hat{h}_{L}}\right)^{2}+\frac{h_{L}}{\hat{h}_{L}}+2}{\sqrt{\frac{h_{L}}{\hat{h}_{L}}+1}}=g \frac{x^{2}+x+2}{4}<g \quad \text { by } \quad 0<x<1 .
$$

Hence by (4.56), we obtain that $u_{-} f^{\prime}\left(h_{-} ; h_{L}\right)-g<0$ when $u_{L}>c_{L}$. This completes the proof of the lemma.

Theorem 4.13. The curve $P^{l}\left(\mathbf{U}_{L}\right)$ defined in (4.47) is strictly decreasing in the $(u, h)$ state plane, while $P^{r}\left(\mathbf{U}_{R}\right)$ defined in (4.48) is strictly increasing in the $(u, h)$ state plane.

Proof. It is sufficient to consider $P^{l}\left(\mathbf{U}_{L}\right)$. The other curve $P^{r}\left(\mathbf{U}_{R}\right)$ can be dealt with in an analogous way.

We need to prove that $\frac{d u}{d h}<0$. Due to $\mathbf{U}=\mathbf{J}\left(z_{R} ; \mathbf{U}_{-}, z_{L}\right)$, we have

$$
\begin{aligned}
h u & =h_{-} u_{-}, \\
\frac{u^{2}}{2}+g\left(h+z_{R}\right) & =\frac{u_{-}^{2}}{2}+g\left(h_{-}+z_{L}\right),
\end{aligned}
$$

where

$$
u_{-}=u_{L}-f\left(h_{-} ; h_{L}\right),
$$


and $f\left(h_{-} ; h_{L}\right)$ is defined in (2.16). By (4.57) and (4.58) we obtain the equations $\tau\left(h, h_{-}\right)=$ 0 and $\varpi\left(u, h_{-}\right)=0$, where

$$
\tau\left(h, h_{-}\right)=\frac{\left(h_{-} u_{-}\right)^{2}}{2 h^{2}}+g\left(h+z_{R}\right)-\frac{u_{-}^{2}}{2}-g\left(h_{-}+z_{L}\right)
$$

and

$$
\varpi\left(u, h_{-}\right)=\frac{u^{2}}{2}+g\left(\frac{h_{-} u_{-}}{u}+z_{R}\right)-\frac{u_{-}^{2}}{2}-g\left(h_{-}+z_{L}\right) .
$$

With the implicit function theorem we obtain

$$
\frac{d h}{d h_{-}}=-\frac{\frac{\partial \tau}{\partial h_{-}}}{\frac{\partial \tau}{\partial h}}=\frac{\frac{\partial \tau}{\partial h_{-}}}{\frac{u^{2}-c^{2}}{h}}
$$

and

so we have

$$
\frac{d u}{d h_{-}}=-\frac{\frac{\partial \varpi}{\partial h_{-}}}{\frac{\partial \varpi}{\partial h}}=-\frac{\frac{\partial \varpi}{\partial h_{-}}}{\frac{u^{2}-c^{2}}{u}},
$$

Lemma 4.49 tells us that

$$
\frac{d u}{d h}=\frac{\frac{d u}{d h_{-}}}{\frac{d h_{-}}{d h_{-}}}=\frac{-u \frac{\partial \varpi}{\partial h_{-}}}{h \frac{\partial \tau}{\partial h_{-}}} .
$$

$$
\frac{\partial \tau}{\partial h_{-}}=\frac{h_{-} u_{-}}{h^{2}} u_{-}\left(u_{-}-h_{-} f^{\prime}\left(h_{-} ; h_{L}\right)\right)+u_{-} f^{\prime}\left(h_{-} ; h_{L}\right)-g<0
$$

and

$$
\frac{\partial \varpi}{\partial h_{-}}=\frac{g}{u}\left(u_{-}-h_{-} f^{\prime}\left(h_{-} ; h_{L}\right)\right)+u_{-} f^{\prime}\left(h_{-} ; h_{L}\right)-g<0 .
$$

Hence we have $\frac{d u}{d h}<0$ from (4.64) by $h>0, u>0$. This completes the proof of the lemma.

Now we define the wave curve

$$
P_{s 0 s}\left(\mathbf{U}_{q}\right)=\left\{\mathbf{U} \mid \mathbf{U}=\mathbf{J}\left(z_{0} ; \mathbf{U}_{+}, z\right) ; \mathbf{U}_{+}=S_{0}^{k}\left(\mathbf{U}_{-}\right) ; \mathbf{U}_{-}=\mathbf{J}\left(z ; \mathbf{U}_{q}, z_{i}\right)\right\},
$$

where $u_{q}^{2} \geq c_{q}^{2}, z_{i} \leq z \leq z_{o}$, as well as $k=1$ when $u_{q}>0$ while $k=2$ when $u_{q}<0$. The state $\mathbf{U}_{-}=\mathbf{J}\left(z ; \mathbf{U}_{q}, z_{i}\right)$ is supersonic while $\mathbf{U}=\mathbf{J}\left(z_{o} ; \mathbf{U}_{+}, z\right)$ is subsonic. Note that this type of resonant wave curve is the general case of the wave curve defined in (4.22). Moreover, we have the following monotonicity lemma for $P_{s 0 s}\left(\mathbf{U}_{q}\right)$.

Lemma 4.14. Assume that $u_{q}^{2} \geq c_{q}^{2}$; we have $\frac{d h}{d z}>0$, while $\frac{d u}{d z}>0$ when $u_{q}>0$ as well as $\frac{d u}{d z}<0$ when $u_{q}<0$ for the wave curves in (4.67).

Proof. It is sufficient to consider the case that $k=1$. The case for $k=2$ can be dealt with in a similar way.

The curve $P_{s 0 s}\left(\mathbf{U}_{q}\right)$ defined in (4.67) is a function in terms of $z$. Note that $\frac{d h}{d z}=$ $\frac{d h}{d h_{-}} \frac{d h_{-}}{d z}$. So we consider $\frac{d h}{d h_{-}}$and $\frac{d h_{-}}{d z}$ in the following. Moreover, we have

$$
h_{q} u_{q}=h_{-} u_{-}=h_{+} u_{+}=h u .
$$

From $\mathbf{U}_{-}=\mathbf{J}\left(z ; \mathbf{U}_{q}, z_{i}\right)$ and $\mathbf{U}=\mathbf{J}\left(z_{o} ; \mathbf{U}_{+}, z\right)$, we respectively have

$$
\frac{u_{q}^{2} h_{q}^{2}}{2 g h_{-}^{2}}+h_{-}+z-\frac{u_{q}^{2}}{2 g}-h_{q}-z_{i}=0
$$


and

$$
\frac{u_{q}^{2} h_{q}^{2}}{2 g h^{2}}+h+z_{o}-\frac{\left(h_{q} u_{q}\right)^{2}}{2 g h_{+}^{2}}-h_{+}-z=0,
$$

where $h_{+}$is defined in (4.32). Similarly to (4.29) and (4.30), we have

$$
z\left(h_{-}\right):=-\frac{h_{q}^{2} u_{q}^{2}}{2 g h_{-}^{2}}-h_{-}+\frac{u_{q}^{2}}{2 g}+h_{q}+z_{q}
$$

and

$$
\frac{d z\left(h_{-}\right)}{d h_{-}}=F_{-}-1>0
$$

Taking (4.29) into (4.70), we introduce a equation $\xi\left(h, h_{-}\right)=0$ where

$$
\xi\left(h, h_{-}\right)=\frac{u_{q}^{2} h_{q}^{2}}{2 g h^{2}}+h+z_{o}-\frac{\left(h_{q} u_{q}\right)^{2}}{2 g h_{+}^{2}}-h_{+}-z\left(h_{-}\right) .
$$

So by the implicit function theorem we have

$$
\frac{d h_{-}}{d h}=-\frac{\frac{\partial \xi}{\partial h}}{\frac{\partial \xi}{\partial h_{-}}}=\frac{F^{2}-1}{\frac{\partial \xi}{\partial h_{-}}}
$$

where $F=\frac{u}{c}$. Using (4.26) and (4.37), we have

$$
\begin{aligned}
\frac{\partial \xi}{\partial h_{-}} & =\frac{\partial \xi}{\partial h_{+}} \frac{d h_{+}}{d h_{-}}+\frac{\partial \xi}{\partial h_{-}} \\
& =\left(F_{+}^{2}-1\right) \frac{d h_{+}}{d h_{-}}-F_{-}^{2}+1 \\
& =-\Theta^{\prime}\left(h_{-}\right)<0 .
\end{aligned}
$$

So we obtain that $\frac{\partial \xi}{\partial h_{-}}<0$ and $\frac{d h_{-}}{d h}>0$. From (4.30) and (4.75), we obtain that $\frac{d h}{d z}=\frac{d h}{d h_{-}} \frac{d h_{-}}{d z}>0$. Since $h u=h_{q} u_{q}, \frac{d u}{d z}=-\frac{u}{h} \frac{d h}{d z}$. Hence $\frac{d u}{d z}<0$ if $u>0$ and vice versa.

In the next section we study the $\mathrm{L}-\mathrm{M}$ and $\mathrm{R}-\mathrm{M}$ curves case by case. The gravity constant $g=9.81$ unless otherwise stated.

4.2. $L-M$ curves with $u_{0 L}>0$ and $u>0$. There are five different types of $\mathrm{L}-\mathrm{M}$ curves. We list the classification for all cases in the following:

- $\mathrm{CASE} I_{L}$ : $\quad z_{\max }<z_{R}$.

- CASE $I I_{L}: \quad z_{\max } \geq z_{R}, u_{L} \leq c_{L} \Longleftrightarrow F_{L}<1$.

- $\mathrm{CASE} I I I_{L}: \quad z_{\max } \geq z_{R}, u_{L}>c_{L} \Longleftrightarrow F_{L}>1, z_{R}<z_{S}<z_{T}$.

- CASE $I V_{L}: \quad z_{\max } \geq z_{R}, u_{L}>c_{L} \Longleftrightarrow F_{L}>1, z_{S}<z_{R}<z_{T}$.

- CASE $V_{L}$ : $\quad z_{\max } \geq z_{R}, u_{L}>c_{L} \Longleftrightarrow F_{L}>1, z_{S}<z_{T}<z_{R}$.

Later we will construct the $\mathrm{L}-\mathrm{M}$ curves for all cases. Before doing this, we consider an example given by Andrianov in [3, (8)]. To match with the assumption $z_{L}<z_{R}$, we reflect the Riemann initial data with respect to $x=0.5$. They become

$$
(z, h, u)= \begin{cases}(1.1,0.1,2.0), & x<0.5 \\ (1.5,1.3,2.0), & x>0.5\end{cases}
$$

with $x \in[0,1]$. Note that $g=2$ in this example. For the given data $c_{L}=\sqrt{0.2}$, we have $u_{L}-c_{L}>0$. From (4.14), we obtain $z_{\max }=1.7912, z_{S}=1.3028$ and $z_{T}=1.7928$. So 
the L-M curve of this example belongs to CASE $I V_{L}$. Reducing $z_{R}$ from 1.5 to 1.3 , we obtain the Riemann initial data for CASE $I I I_{L}$. Later these Riemann initial data will be used to give examples for the Riemann solutions.

4.2.1. CASE $I_{L}: z_{\max }<z_{R}$. This is the case when the jump of the bottom step is too high as compared with the inflow state $\mathbf{U}_{-}$of the stationary wave, which is connected to $\mathbf{U}_{L}$ by a negative 1-wave. Mathematically we say that there is no solution to $J\left(z_{R} ; \mathbf{U}_{-}, z_{L}\right)$ for any $\mathbf{U}_{-} \in T_{1}\left(\mathbf{U}_{L}\right)$ with a negative speed 1 -wave. This was proved in Lemma 4.2 .

Generally there are two different subcases for this case:

- $h_{R}=0$.

- $u_{0 R}>0$.

We have the following two Riemann problems:

$$
\begin{gathered}
h_{t}+(h u)_{x}=0, \\
(h u)_{t}+\left(h u^{2}+\frac{g h^{2}}{2}\right)_{x}=0 . \\
(h, u)(x, 0)= \begin{cases}\left(h_{L}, u_{L}\right), & x<x_{0}, \\
\left(h_{L},-u_{L}\right), & x>x_{0} .\end{cases} \\
(h, u)(x, 0)= \begin{cases}(0,0), & x<x_{0}, \\
\left(h_{R}, u_{R}\right), & x>x_{0} .\end{cases}
\end{gathered}
$$

We find that when $h_{R}=0$ or $u_{0 R}>0$, the solution of the Riemann problem can be split into two parts. One part is the solution to the Riemann problem (4.77) and (4.78) in the region $x<x_{0}$. The other part is the solution to Riemann problem (4.77) and (4.79) in the region $x>x_{0}$. Note that if $h_{R}=0$, the solution to (4.77) and (4.79) is $h=0$ and $u=0$ for $(x, t) \in \mathbb{R} \times \mathbb{R}^{+}$. The wave configuration of $u_{0 R}>0$ can be seen in Figure 3 , The wave configuration of $h_{R}=0$ can refer to Figure 4 .

Here we give two examples to illustrate our construction. The first example has the wave configuration $H_{1}$. The results are shown in Figure 20, where $z_{\max }=3.5769<z_{R}=$ 4.7. The second example has the wave configuration $H_{2}$. The results are shown in Figure 21. where $z_{\max }=2.4724<z_{R}=4.0$.
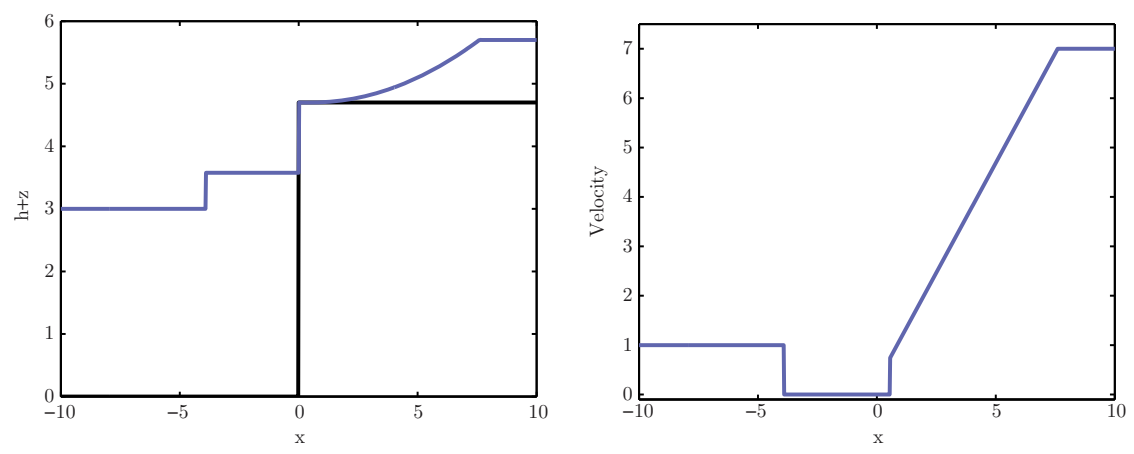

FIG. 20. Left: The water free surface $h+z$ at $t=0.75$. Right: The velocity. The Riemann initial data are $\left(z_{L}, h_{L}, u_{L}\right)=(0,3,1)$ when $x<0$ and $\left(z_{R}, h_{R}, u_{R}\right)=(4.7,1.0,7.0)$ when $x>0$. 



Fig. 21. Left: The water free surface $h+z$ at $t=0.75$. Right: The velocity. The Riemann initial data are $\left(z_{L}, h_{L}, u_{L}\right)=(0,3.0,-1.0)$ when $x<0$ and $\left(z_{R}, h_{R}, u_{R}\right)=(4.0,0,0)$ when $x>0$.

4.2.2. CASE $I I_{L}: z_{\max } \geq z_{R}, u_{L} \leq c_{L}$. In this case the $\mathrm{L}-\mathrm{M}$ curve consists of three segments which are defined as follows:

$$
\begin{aligned}
& P_{1}^{l}\left(\mathbf{U}_{L}\right)=\left\{\mathbf{U} \mid \mathbf{U} \in T_{1}\left(\mathbf{U}_{L}\right) \text { with } u<0\right\}, \\
& P_{2}^{l}\left(\mathbf{U}_{L}\right)=\left\{\mathbf{U} \mid \mathbf{U}=\mathbf{J}\left(z_{R} ; \mathbf{U}_{-}, z_{L}\right) \text { and } \mathbf{U}_{-} \in T_{1}\left(\mathbf{U}_{L}\right) \text { with } 0<u_{-}<\tilde{u}_{c}, 0<u<u_{c}\right\}, \\
& P_{3}^{l}\left(\mathbf{U}_{L}\right)=\left\{\mathbf{U} \mid \mathbf{U} \in T_{1}\left(\mathbf{U}_{c}\right) \text { with } u>u_{c}\right\},
\end{aligned}
$$

where $\mathbf{U}_{c}=\mathbf{J}\left(z_{R} ; \tilde{\mathbf{U}}_{c}, z_{L}\right), \tilde{\mathbf{U}}_{c} \in T_{1}\left(\mathbf{U}_{L}\right)$ which is defined in 4.15).

The continuity of the three segments is obvious. According to Theorem 4.13, the segment $P_{2}^{l}\left(\mathbf{U}_{L}\right)$ is strictly decreasing in the $(u, h+z)$ space. Also the segments $P_{1}^{l}\left(\mathbf{U}_{L}\right)$ and $P_{3}^{l}\left(\mathbf{U}_{L}\right)$ are strictly decreasing in the $(u, h+z)$ space due to Lemma 2.1. So the $\mathrm{L}-\mathrm{M}$ curve $\bigcup_{k=1}^{3} P_{k}^{l}\left(\mathbf{U}_{L}\right)$ is strictly decreasing in the $(u, h+z)$ space.

We define

$$
u_{0 L}^{*}=3 u_{c}
$$

If $u_{0 L}^{*}>u_{0 R}$, there is a unique intersection point between the $\mathrm{L}-\mathrm{M}$ curve and the $\mathrm{R}-$ M curve. If the intersection point lies on the segment $P_{2}^{l}\left(\mathbf{U}_{L}\right)$, the solution has the wave configurations $A$; see Figure 2. Here we use an example given by Alcrudo and Benkhaldoun in 5 to illustrate the corresponding L-M curve and the exact free surface of the fluids, as well as the Froude number in Figure 22. If the intersection point lies on the segment $P_{3}^{l}\left(\mathbf{U}_{L}\right)$, the solution has the wave configuration $B$. An example is shown in Figure 23. We observe that the Froude number is greater than 1 when the water goes across the bottom jump.

Otherwise if $u_{0 L}^{*}<u_{0 R}$ and $h_{R}>0$, the Riemann solution contains a dry bed state and behaves in the manner of the wave configuration $B_{v}$; see Figure 7 The example for $h_{R}>0$ is shown in Figure 24. The example for $h_{R}=0$ is shown in Figure 25. 

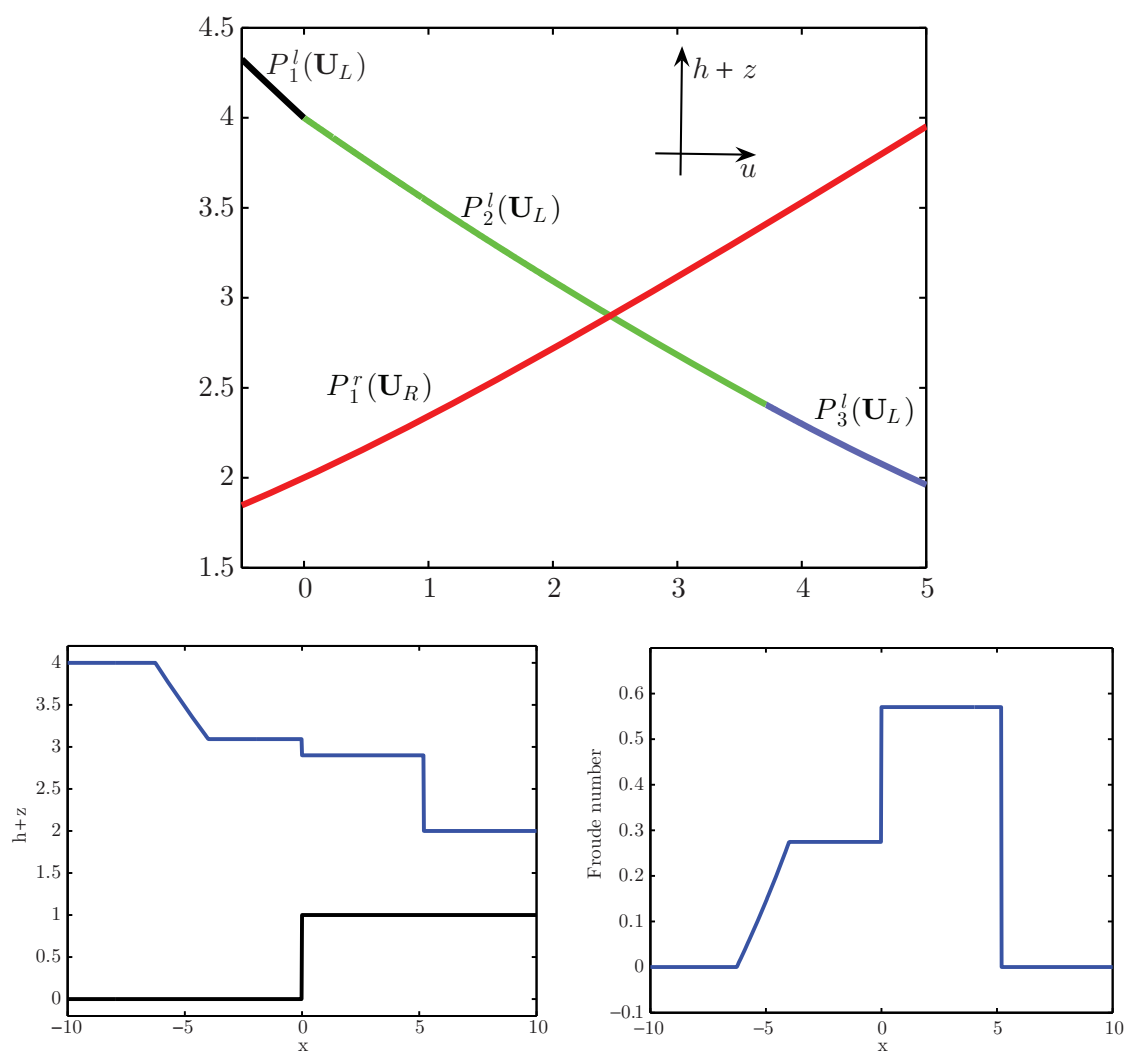

FIG. 22. Top: L-M curve $\bigcup_{k=1}^{3} P_{k}^{l}\left(\mathbf{U}_{L}\right)$. Bottom left: The water free surface $h+z$ at $t=1.0$. Bottom right: The Froude number. The Riemann initial data are $\left(z_{L}, h_{L}, u_{L}\right)=(0.0,4.0,0.0)$ when $x<0$ and $\left(z_{R}, h_{R}, u_{R}\right)=(1.0,1.0,0.0)$ when $x>0$.

4.2.3. CASE III $I_{L}: z_{\max } \geq z_{R}, u_{L}>c_{L}, z_{R}<z_{S}<z_{T}$. In this case the L-M curve consists of the following four parts:

$$
\begin{aligned}
& P_{1}^{l}\left(\mathbf{U}_{L}\right)=\left\{\mathbf{U} \mid \mathbf{U} \in T_{1}\left(\mathbf{U}_{L}\right) \text { with } u<0\right\}, \\
& P_{2}^{l}\left(\mathbf{U}_{L}\right)=\left\{\mathbf{U} \mid \mathbf{U}=\mathbf{J}\left(z_{R} ; \mathbf{U}_{-}, z_{L}\right) \text { and } \mathbf{U}_{-} \in S_{1}^{-}\left(\mathbf{U}_{L}\right) \text { with } 0<u_{-}<\hat{u}_{L}, 0<u<\overline{\hat{u}}_{L}\right\}, \\
& P_{3}^{l}\left(\mathbf{U}_{L}\right)=\left\{\mathbf{U} \mid \mathbf{U}=\mathbf{J}\left(z_{R} ; \mathbf{U}_{+}, z\right) ; \mathbf{U}_{+}=S_{1}^{0}\left(\mathbf{U}_{-}\right) ; \mathbf{U}_{-}=\mathbf{J}\left(z ; \mathbf{U}_{L}, z_{L}\right), z_{L} \leq z \leq z_{R}\right\}, \\
& P_{4}^{l}\left(\mathbf{U}_{L}\right)=\left\{\mathbf{U} \mid \mathbf{U} \in T_{1}\left(\overline{\mathbf{U}}_{L}\right) \text { with } u>\hat{\bar{u}}_{L}\right\},
\end{aligned}
$$

where $\overline{\hat{\mathbf{U}}}_{L}=\mathbf{J}\left(z_{R} ; \hat{\mathbf{U}}_{L}, z_{L}\right)$ and $\hat{\mathbf{U}}_{L}=S_{1}^{0}\left(\mathbf{U}_{L}\right)$, while $\hat{\mathbf{U}}_{L}=S_{1}^{0}\left(\overline{\mathbf{U}}_{L}\right)$ and $\overline{\mathbf{U}}_{L}=$ $\mathbf{J}\left(z_{R} ; \mathbf{U}_{L}, z_{L}\right)$. Due to $z_{L}<z_{R}$, Lemma 4.14 tells us that $h$ is increasing while $u$ is decreasing when $z$ varies monotonically from $z_{L}$ to $z_{R}$. So $\overline{\hat{h}}_{L}<\hat{\bar{h}}_{L}$ and $\overline{\hat{u}}_{L}>\hat{\bar{u}}_{L}$. As shown in Figure 26, the L-M curve is folding in the $(u, h+z)$ state space.

We define

$$
u_{0 L}^{*}=\bar{u}_{L}+2 \bar{c}_{L}
$$



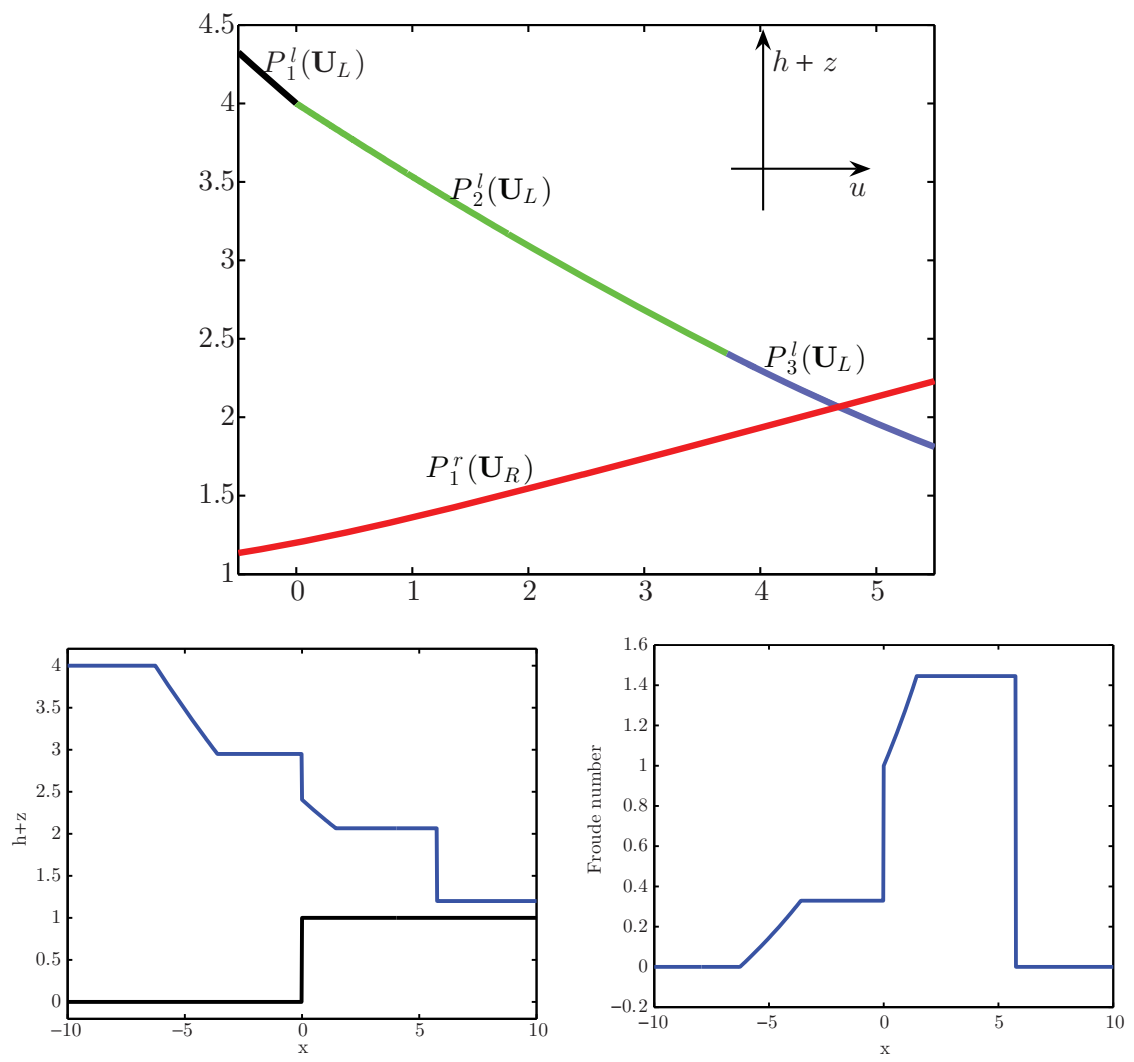

Fig. 23. Top: L-M curve $\bigcup_{k=1}^{3} P_{k}^{l}\left(\mathbf{U}_{L}\right)$. Bottom left: The water free surface $h+z$ at $t=1.0$. Bottom right: The Froude number. The Riemann initial data are $\left(z_{L}, h_{L}, u_{L}\right)=(0.0,4.0,0.0)$ when $x<0$ and $\left(z_{R}, h_{R}, u_{R}\right)=(1.0,0.2,0.0)$ when $x>0$.

Note that if $u_{0 L}^{*}>u_{0 R}$, there are intersection points between the L-M curve and the $\mathrm{R}-\mathrm{M}$ curve. If the intersection point lies on the segment $P_{2}^{l}\left(\mathbf{U}_{L}\right)$, the solution is in the pattern of the wave configuration $A$. This is analogous to the CASE $I I_{L}$. If the intersection point lies on the segment $P_{3}^{l}\left(\mathbf{U}_{L}\right)$, the solution has the wave configuration $C$, while if the intersection point lies on the segment $P_{4}^{l}\left(\mathbf{U}_{L}\right)$, the solution has the wave configuration $D$.

Due to the fact that the $\mathrm{L}-\mathrm{M}$ curve is folding in the $(u, h+z)$ state space, if the intersection point lies on the segment $P_{3}^{l}\left(\mathbf{U}_{L}\right)$, we can also find two other intermediate states lying on the segments $P_{2}^{l}\left(\mathbf{U}_{L}\right)$ and $P_{4}^{l}\left(\mathbf{U}_{L}\right)$ respectively. So for one set of given initial data, there are three solutions with wave configurations $A, C$ and $D$ respectively. An example with $g=2.0$ is shown in Figure 26. An example for $g=9.81$ is shown in Figure 27. 

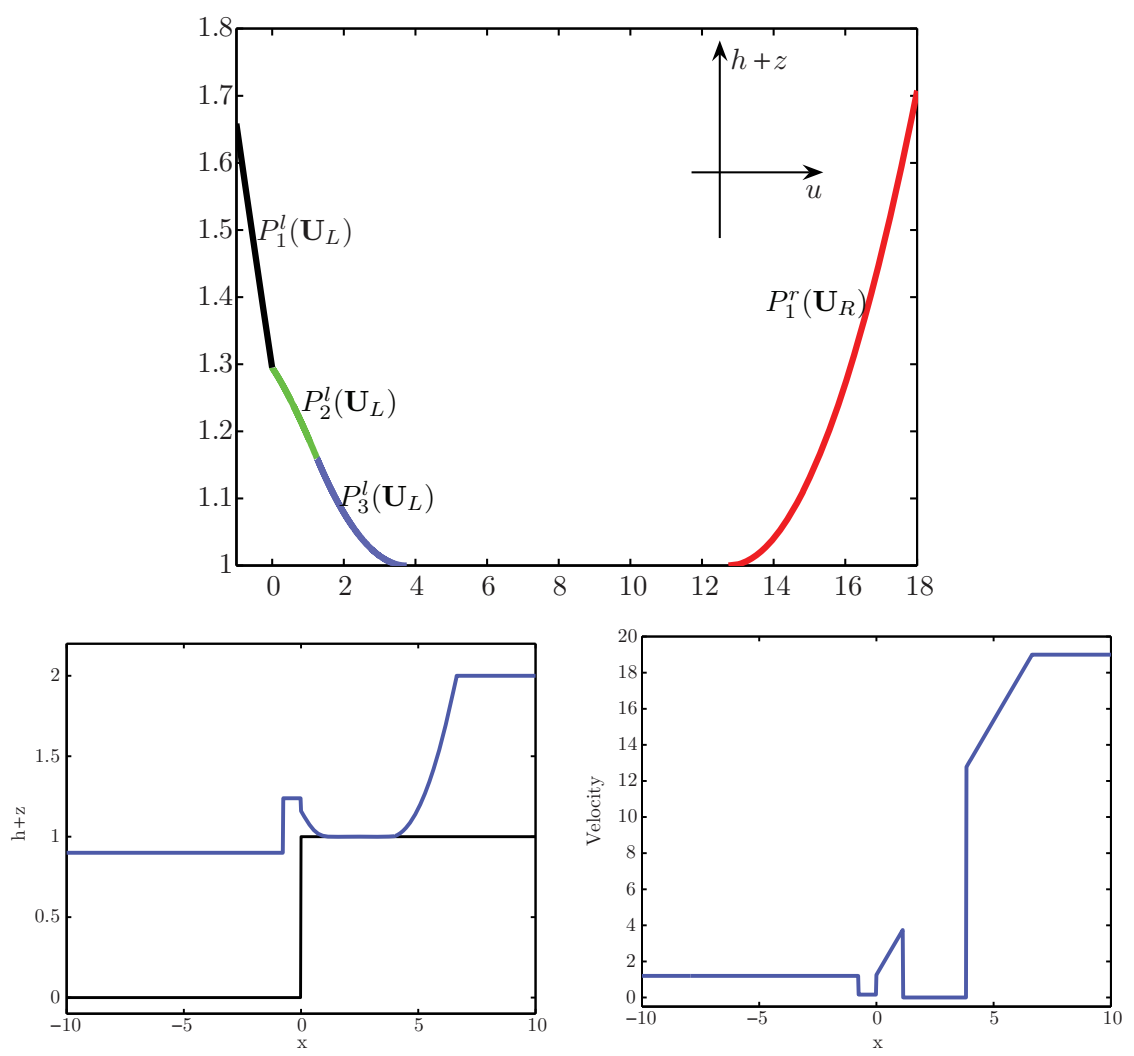

Fig. 24. Top: L-M curve $\bigcup_{k=1}^{3} P_{k}^{l}\left(\mathbf{U}_{L}\right)$. Bottom left: The water free surface $h+z$ at $t=0.3$. Bottom right: The velocity. The Riemann initial data are $\left(z_{L}, h_{L}, u_{L}\right)=(0,0.9,1.2)$ when $x<0$ and $\left(z_{R}, h_{R}, u_{R}\right)=(1.0,1.0,19.0)$ when $x>0$.

Moreover, if $u_{0 L}^{*}<u_{0 R}$, the solution with the wave configuration $D_{v}$ occurs. An example for $h_{R}>0$ is shown in Figure 28. An example for $h_{R}=0$ is shown in Figure 29. Note that the computational region for these two examples is $[-10,10]$ and $g=2.0$.

4.2.4. CASE $I V_{L}: z_{\max } \geq z_{R}, u_{L}>c_{L}, z_{S}<z_{R}<z_{T}$. In this case the L-M curve consists of six parts. They are defined as follows:

$$
\begin{aligned}
& P_{1}^{l}\left(\mathbf{U}_{L}\right)=\left\{\mathbf{U} \mid \mathbf{U} \in T_{1}\left(\mathbf{U}_{L}\right) \text { with } u<0\right\}, \\
& P_{2}^{l}\left(\mathbf{U}_{L}\right)=\left\{\mathbf{U} \mid \mathbf{U}=\mathbf{J}\left(z_{R} ; \mathbf{U}_{-}, z_{L}\right) \text { and } \mathbf{U}_{-} \in S_{1}^{-}\left(\mathbf{U}_{L}\right) \text { with } u_{-}<\tilde{u}_{c}^{L}, u<u_{c_{2}}\right\}, \\
& P_{3}^{l}\left(\mathbf{U}_{L}\right)=\left\{\mathbf{U} \mid \mathbf{U}=\mathbf{J}\left(z_{R} ; \mathbf{U}_{+}, z\right) ; \mathbf{U}_{+}=S_{0}\left(\mathbf{U}_{-}\right) ; \mathbf{U}_{-}=\mathbf{J}\left(z ; \mathbf{U}_{L}, z_{L}\right), z_{c} \leq z \leq z_{R}\right\}, \\
& P_{4}^{l}\left(\mathbf{U}_{L}\right)=\left\{\mathbf{U} \mid \mathbf{U} \in T_{1}\left(\overline{\mathbf{U}}_{L}\right) \text { with } u>\hat{\bar{u}}_{L}\right\}, \\
& P_{5}^{l}\left(\mathbf{U}_{L}\right)=\left\{\mathbf{U} \mid \mathbf{U} \in T_{1}\left(\mathbf{U}_{c_{2}}\right) \text { with } u>u_{c_{2}}\right\}, \\
& P_{6}^{l}\left(\mathbf{U}_{L}\right)=\left\{\mathbf{U} \mid \mathbf{U} \in T_{1}\left(\mathbf{U}_{c_{3}}\right) \text { with } u>u_{c_{3}}\right\},
\end{aligned}
$$

where $\left(\tilde{h}_{c}^{L}, \tilde{u}_{c}^{L}\right)$ and $\mathbf{U}_{c_{2}}$ are defined in Remark 4.10, while $\mathbf{U}_{c_{3}}$ is defined in Remark 4.11. Compared with the $\mathrm{L}-\mathrm{M}$ curve in CASE $I I I_{L}$, it seems that the boundary state 

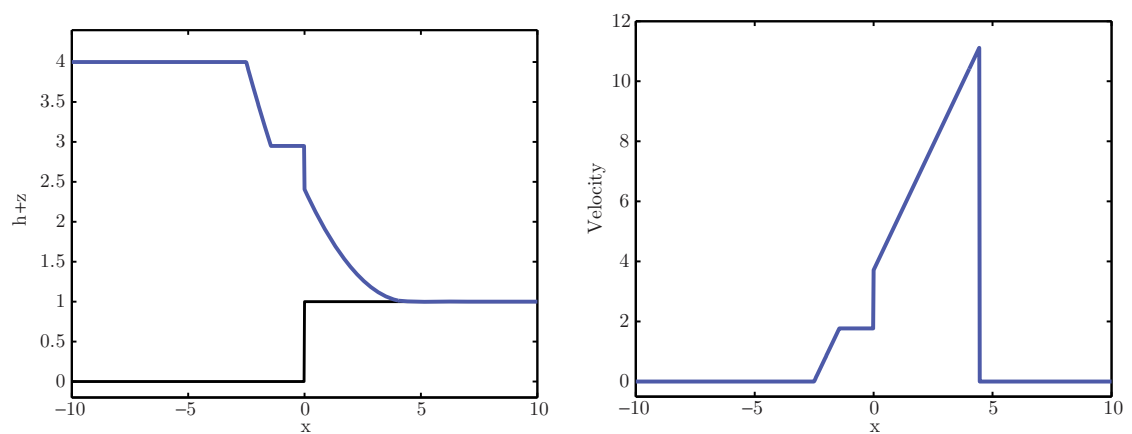

Fig. 25. Left: The water free surface $h+z$ at $t=0.4$. Right: The velocity. The Riemann initial data are $\left(z_{L}, h_{L}, u_{L}\right)=(0,4.0,0.0)$ when $x<0$ and $\left(z_{R}, h_{R}, u_{R}\right)=(1.0,0.0,0.0)$ when $x>0$.

$\hat{\mathbf{U}}_{L}$ bifurcates into two segments $P_{5}^{l}\left(\mathbf{U}_{L}\right)$ and $P_{6}^{l}\left(\mathbf{U}_{L}\right)$. Generally the $\mathrm{L}-\mathrm{M}$ curve in this case consists of three branches $P_{1}^{l}\left(\mathbf{U}_{L}\right) \cup P_{2}^{l}\left(\mathbf{U}_{L}\right) \cup P_{5}^{l}\left(\mathbf{U}_{L}\right), P_{3}^{l}\left(\mathbf{U}_{L}\right) \cup P_{6}^{l}\left(\mathbf{U}_{L}\right)$ and $P_{4}^{l}\left(\mathbf{U}_{L}\right)$; see Figure 31. Apparently, if the intersection points belong to $P_{3}^{l}\left(\mathbf{U}_{L}\right), P_{4}^{l}\left(\mathbf{U}_{L}\right), P_{5}^{l}\left(\mathbf{U}_{L}\right)$ or $P_{6}^{l}\left(\mathbf{U}_{L}\right)$, there are three possible solutions for the same initial data.

Analogously to CASE $I I I_{L}$, the wave configurations $A, C$ and $D$ are related to the segments $P_{2}^{l}\left(\mathbf{U}_{L}\right), P_{3}^{l}\left(\mathbf{U}_{L}\right)$ and $P_{4}^{l}\left(\mathbf{U}_{L}\right)$ respectively. Besides, the wave configuration $B$ is related to the segment $P_{5}^{l}\left(\mathbf{U}_{L}\right)$, while the wave configuration $E$, see Figure 12] is related to the segment $P_{6}^{l}\left(\mathbf{U}_{L}\right)$.

An example of the three solutions with the wave configurations $A, C$ and $D$ is presented in Figure 31. As we have mentioned, this example comes from Andrianov 3 . However, he omitted the solution with the wave configuration $C$ due to the fact that it contains a resonant wave $S 0 S\left(\mathbf{U}_{\mathbf{L}}\right)$; see [9]. We reduce $h_{R}$ in (4.76) from 1.3 to 0.45 . There are still three solutions but with the wave configurations $B, E$ and $F$; see Figure 30.

We define

$$
u_{0 L}^{*, 1}=3 u_{c_{2}}, \quad u_{0 L}^{*, 2}=3 u_{c_{3}}, \quad u_{0 L}^{*, 3}=\bar{u}_{L}+2 \bar{c}_{L}
$$

Note that if $u_{0 L}^{*, 1}<u_{0 R}$, a solution with the wave configuration $B_{v}$ occurs. Similarly, if $u_{0 L}^{*, 2}<u_{0 R}$, a solution with the wave configuration $E_{v}$ occurs, while if $u_{0 L}^{*, 3}<u_{0 R}$, the solution with the wave configuration $D_{v}$ occurs. The example of these three types of solutions with $h_{R}>0$ can be found in Figure 32. The example for the case when $h_{R}=0$ is shown in Figure 33 .

4.2.5. CASE $V_{L}: z_{\max } \geq z_{R}, u_{L}>c_{L}, z_{S}<z_{T}<z_{R}$. When $z_{T}<z_{S}<z_{R}$, Lemma 4.9 tells us the segment $P_{3}^{l}\left(\mathbf{U}_{L}\right)$ in (4.22) fails to exist. Therefore the $\mathrm{L}-\mathrm{M}$ curve in this case consists of three segments, which are defined as follows:

$$
\begin{aligned}
& P_{1}^{l}\left(\mathbf{U}_{L}\right)=\left\{\mathbf{U} \mid \mathbf{U} \in T_{1}\left(\mathbf{U}_{L}\right) \text { with } u<0\right\} \\
& P_{2}^{l}\left(\mathbf{U}_{L}\right)=\left\{\mathbf{U} \mid \mathbf{U}=\mathbf{J}\left(z_{R} ; \mathbf{U}_{-}, z_{L}\right) \text { and } \mathbf{U}_{-} \in S_{1}^{-}\left(\mathbf{U}_{L}\right) \text { with } u_{-}<\tilde{u}_{c}^{L}, u<u_{c_{2}}\right\} \\
& P_{3}^{l}\left(\mathbf{U}_{L}\right)=\left\{\mathbf{U} \mid \mathbf{U} \in T_{1}\left(\mathbf{U}_{c_{2}}\right) \text { with } u>u_{c_{2}}\right\} .
\end{aligned}
$$



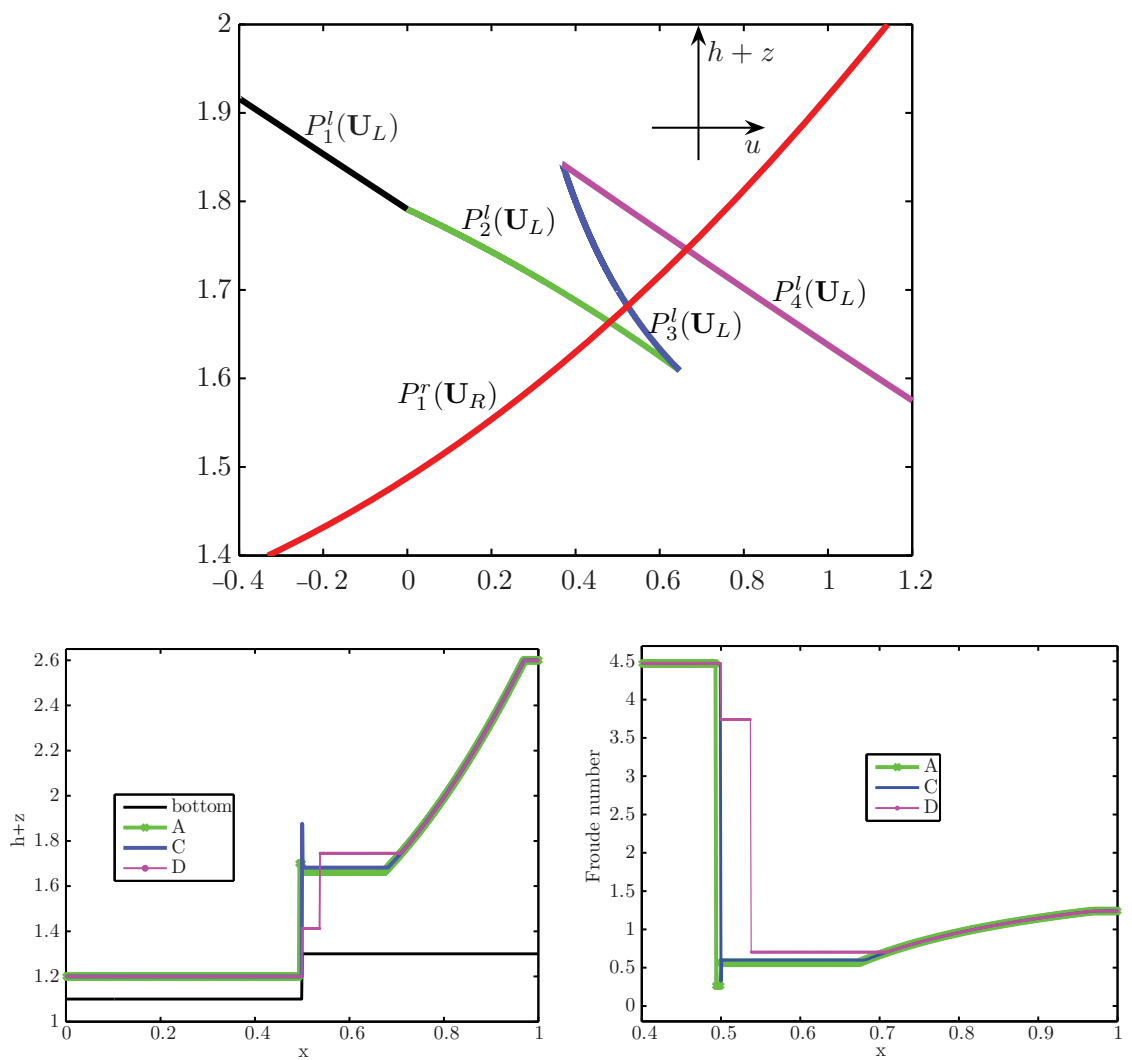

Fig. 26. Top: L-M curve $\bigcup_{k=1}^{4} P_{k}^{l}\left(\mathbf{U}_{L}\right)$. Bottom left: The water free surface $h+z$ at $t=0.13$. Bottom right: The Froude number. The Riemann initial data are given in (4.76) but with $z_{R}=1.3$.

Note that the L-M curve in this case is just one branch of the $\mathrm{L}-\mathrm{M}$ curve in CASE $I V_{L}$ and it is decreasing and continuous. We define

$$
u_{0 L}^{*}=3 u_{c_{2}} .
$$

We observe that if $u_{0 L}^{*}>u_{0 R}$, there is an intersection point between the $\mathrm{L}-\mathrm{M}$ curve and the $\mathrm{R}-\mathrm{M}$ curve. If the intersection point lies on $P_{2}^{l}\left(\mathbf{U}_{L}\right)$, the solution has the wave configuration $A$. An example is shown in Figure 34. In the other case when the intersection point lies on $P_{2}^{l}\left(\mathbf{U}_{L}\right)$, the solution has the wave configuration $B$. An example is shown in Figure 35. In the other case when $u_{0 L}^{*}<u_{0 R}$, the solution with the wave configuration $B_{v}$ occurs. An example with $h_{R}>0$ is shown in Figure 36. An example with $h_{R}=0$ is shown in Figure 37 .

4.3. $R-M$ curves with $u_{0 R}<0$ and $u<0$. Generally, there are two possible cases for the $\mathrm{R}-\mathrm{M}$ curves if $z_{L}<z_{R}$. Remember that we do not have to consider $z_{R}>z_{L}$ because these cases can be deduced by symmetry of solutions.

- CASE $I_{R}: u_{R}+c_{R} \geq 0 \Longleftrightarrow F_{R}>-1$. 

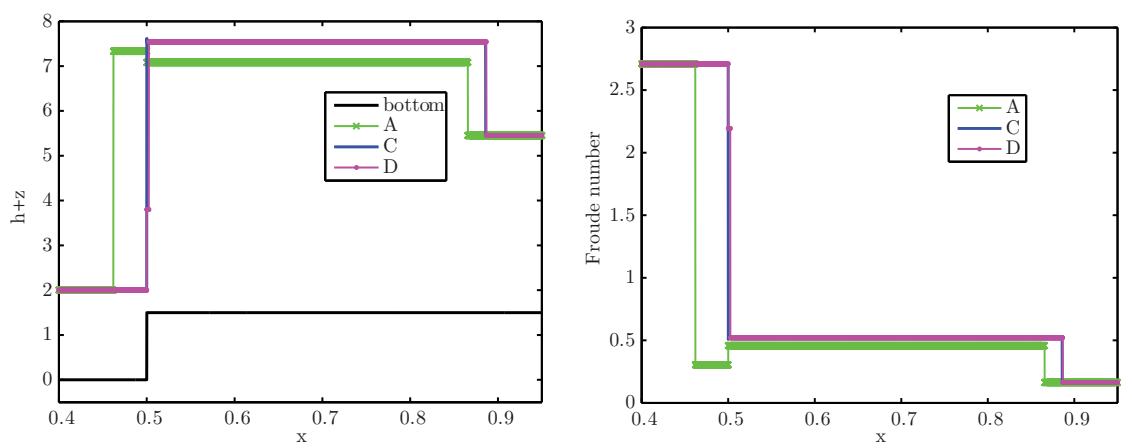

FIG. 27. Left: The water free surface $h+z$ at $t=0.03$. Right: The Froude number. The Riemann initial data are $\left(z_{L}, h_{L}, u_{L}\right)=$ $(0,2.0,12.0)$ when $x<0.5$ and $\left(z_{R}, h_{R}, u_{R}\right)=(1.5,3.9524,1.0142)$ when $x>0.5$. The computational region is [0.4, 0.95].


FIG. 28. Left: The water free surface $h+z$ at $t=0.4$. Right: The velocity. The Riemann initial data are $\left(z_{L}, h_{L}, u_{L}\right)=(0,0.7,4.0)$ when $x<0$ and $\left(z_{R}, h_{R}, u_{R}\right)=(0.8,0.2,14.0)$ when $x>0$.

- CASE $I I_{R}: u_{R}+c_{R}<0 \Longleftrightarrow F_{R}<-1$.

We define $h_{R}^{\max }$ as the solution to equation

$$
0=u_{R}+f\left(h_{R}^{\max } ; h_{R}\right)
$$

The calculation procedure for $h_{R}^{\max }$ is similar to $h_{L}^{\max }$ in (4.13). We intend to study these two cases of the $\mathrm{R}-\mathrm{M}$ curves in the following.

4.3.1. CASE $I_{R}: u_{R}+c_{R} \geq 0$. In this case the sonic state can only appear on the right side of the initial discontinuity located at $x=x_{0}$ due to the fact that $z_{L}<z_{R}$, i.e. $\mathbf{U}_{c} \in T_{2}\left(\mathbf{U}_{R}\right)$. According to Remark 3.3, $\mathbf{U}=\mathbf{J}\left(z_{R} ; \mathbf{U}_{c}, z_{L}\right)$ has two solutions. One is supersonic, and the other one is subsonic. We use $\overline{\mathbf{U}}_{c}^{*}=\mathbf{J}\left(z ; \mathbf{U}_{c}, z_{R}\right)$ to denote the supersonic one and $\overline{\mathbf{U}}_{c}=\mathbf{J}\left(z ; \mathbf{U}_{c}, z_{R}\right)$ to denote the subsonic one. 

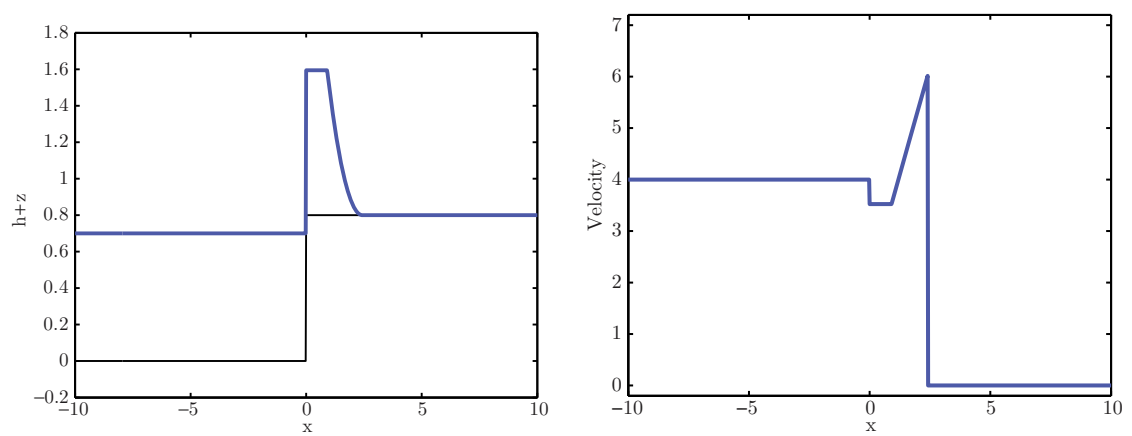

FIG. 29. Left: The water free surface $h+z$ at $t=0.4$. Right: The velocity. The Riemann initial data are $\left(z_{L}, h_{L}, u_{L}\right)=(0,0.7,4.0)$ when $x<0$ and $\left(z_{R}, h_{R}, u_{R}\right)=(0.8,0,0)$ when $x>0$.
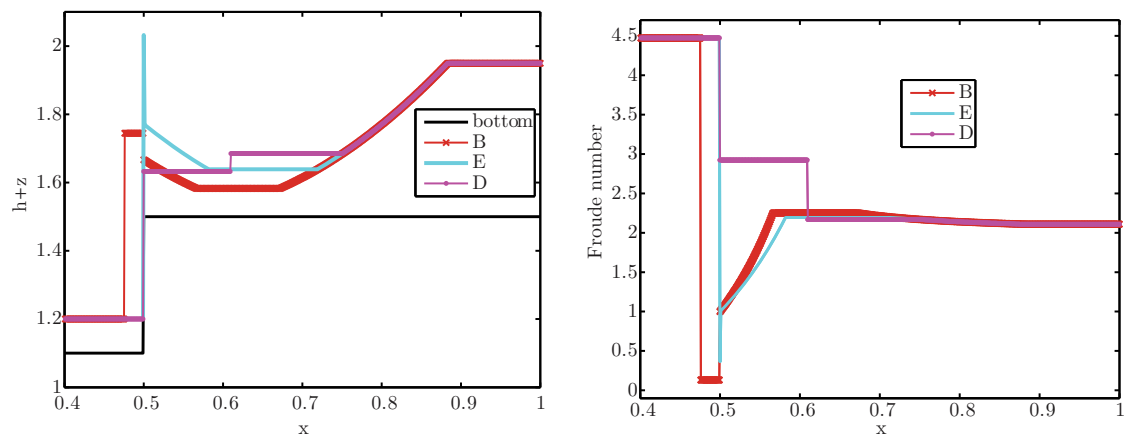

FIG. 30. Left: The water free surface $h+z$ at $t=1$. Right: The Froude number. The Riemann initial data are given in 4.76) but with $h_{R}=0.45$.

The $\mathrm{R}-\mathrm{M}$ curve in this case consists of four segments, which are defined in the following:

$$
\begin{aligned}
& P_{1}^{r}\left(\mathbf{U}_{R}\right)=\left\{\mathbf{U} \mid \mathbf{U} \in T_{2}\left(\mathbf{U}_{R}\right) \text { with } u>0\right\} \\
& P_{2}^{r}\left(\mathbf{U}_{R}\right)=\left\{\mathbf{U} \mid \mathbf{U}=\mathbf{J}\left(z_{L} ; \mathbf{U}_{-}, z_{R}\right) \text { and } \mathbf{U}_{-} \in T_{2}\left(\mathbf{U}_{R}\right) \text { with } u_{c}<u_{-}<0, \bar{u}_{c}<u<0\right\}, \\
& P_{3}^{r}\left(\mathbf{U}_{R}\right)=\left\{\mathbf{U} \mid \mathbf{U}=\mathbf{J}\left(z_{L} ; \mathbf{U}_{+}, z\right) ; \mathbf{U}_{+}=S_{2}^{0}\left(\mathbf{U}_{-}\right) ; \mathbf{U}_{-}=\mathbf{J}\left(z ; \mathbf{U}_{c}, z_{R}\right), z_{L} \leq z \leq z_{R}\right\}, \\
& P_{4}^{r}\left(\mathbf{U}_{L}\right)=\left\{\mathbf{U} \mid \mathbf{U} \in T_{2}\left(\overline{\mathbf{U}}_{c}^{*}\right) \text { with } u<\overline{\bar{u}}_{c}\right\} .
\end{aligned}
$$

We have to remember that the state $\mathbf{U}=\mathbf{J}\left(z_{R} ; \mathbf{U}_{+}, z\right)$ is subsonic, and the state $\mathbf{U}_{-}=$ $\mathbf{J}\left(z ; \mathbf{U}_{c}, z_{L}\right)$ is supersonic for the segment $P_{3}^{r}\left(\mathbf{U}_{R}\right)$.

The continuity of the three segments is obvious. From Lemma 2.1 the segments of $P_{1}^{r}\left(\mathbf{U}_{R}\right)$ and $P_{4}^{r}\left(\mathbf{U}_{R}\right)$ are strictly increasing in the $(u, h+z)$ space. Theorem 4.13 indicates that $P_{2}^{r}\left(\mathbf{U}_{R}\right)$ is strictly increasing in the $(u, h+z)$ space. Moreover, due to the fact that $z_{L}<z_{R}$, Lemma 4.14 tells us that $h$ and $u$ are strictly decreasing when $z$ varies from $z_{R}$ to $z_{L}$. So the segment $P_{3}^{r}\left(\mathbf{U}_{R}\right)$ is strictly increasing in the $(u, h+z)$ space. In summary, the $\mathrm{R}-\mathrm{M}$ curve in this case is continuous and strictly increasing in the $(u, h+z)$ space. 

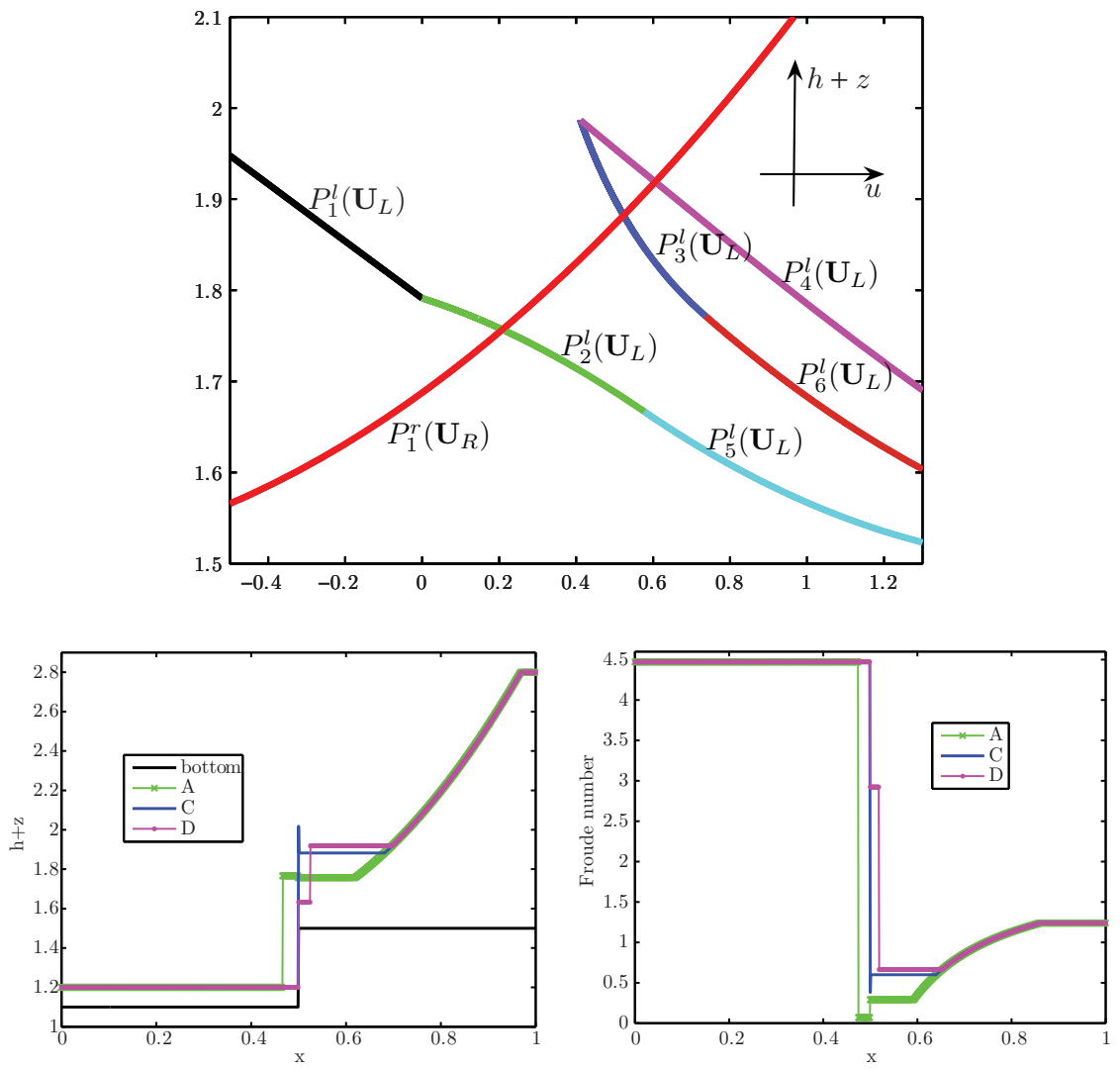

FIG. 31. Top: L-M curve $\bigcup_{k=1}^{6} P_{k}^{l}\left(\mathbf{U}_{L}\right)$. Bottom left: The water free surface $h+z$ at $t=1$. Bottom right: the Froude number. The Riemann initial data are given in 4.76).

We define

$$
u_{0 R}^{*}=u_{c}^{*}-2 c_{c}^{*} .
$$

Note that if $h_{L}>0$ and $u_{0 L}>u_{0 R}^{*}$, the curve $P_{1}^{l}\left(\mathbf{U}_{L}\right)$ and the $\mathrm{R}-\mathrm{M}$ curve always have an intersection point. If the intersection point lies on $P_{2}^{r}\left(\mathbf{U}_{R}\right)$, the solution has the wave configuration $A^{T}$; see Figure 16. An example can be found in Figure 38. If the intersection point lies on $P_{3}^{r}\left(\mathbf{U}_{R}\right)$, the solution has the wave configuration $F$; see Figure 9. An example is shown in Figure 39. We can see that the resonant wave occurs around $x=0.5$. Similarly, if the intersection point lies on $P_{4}^{r}\left(\mathbf{U}_{R}\right)$, the solution has the wave configuration $G$; see Figure 14. An example is shown in Figure 40.

However if $u_{0 L}<u_{0 R}^{*}$ and $h_{L}>0$, the Riemann solution contains a dry bed state since there is no intersection point between $P_{1}^{l}\left(\mathbf{U}_{L}\right)$ and the $\mathrm{R}-\mathrm{M}$ curve; see Figure 41, Specifically, the solution has the wave configuration $G_{v}$; see Figure 15. An example with $h_{L}>0$ can be found in Figure 41. An example with $h_{L}<0$ is shown in Figure 41, Here all of the examples are in the interval $[0,1]$. 

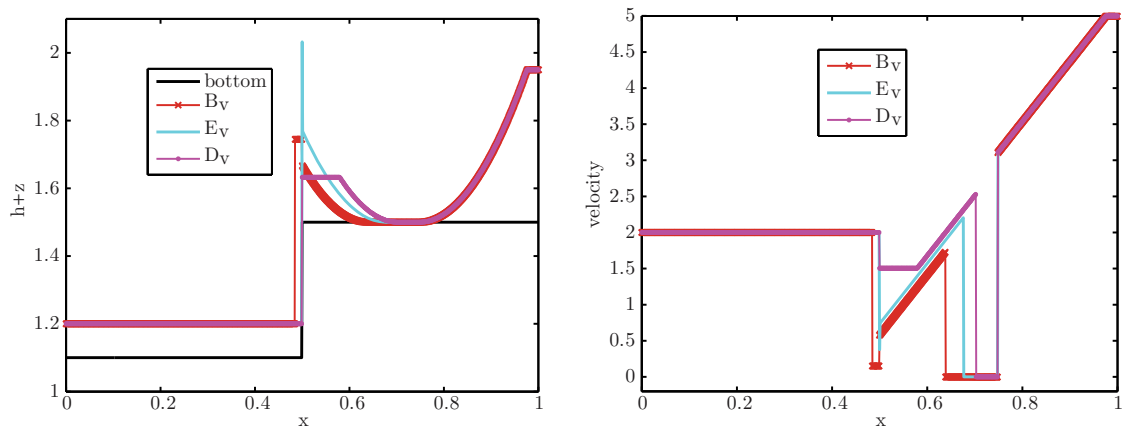

FIG. 32. Left: The water free surface $h+z$ at $t=0.08$. Right: The velocity. The Riemann initial data are $\left(z_{L}, h_{L}, u_{L}\right)=(1.1,0.1,2.0)$ when $x<0.5$ and $\left(z_{R}, h_{R}, u_{R}\right)=(1.5,0.45,5.0)$ when $x>0.5$.
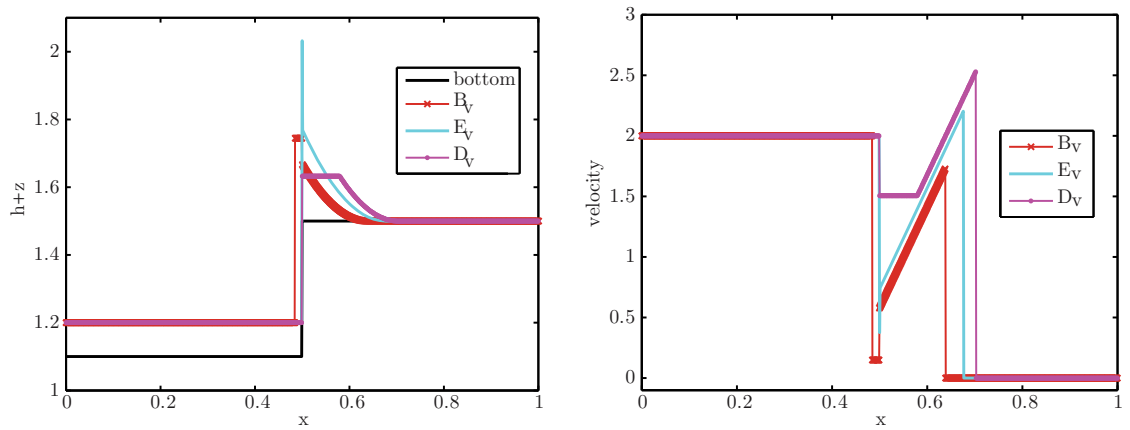

Fig. 33. Left: The water free surface $h+z$ at $t=0.08$. Right: The velocity. The Riemann initial data are $\left(z_{L}, h_{L}, u_{L}\right)=(1.1,0.1,2.0)$ when $x<0.5$ and $\left(z_{R}, h_{R}, u_{R}\right)=(1.5,0,0)$ when $x>0.5$.

4.3.2. CASE $I I_{R}: u_{R}+c_{R}<0$. In this case the $\mathrm{R}-\mathrm{M}$ curve also consists of four segments, which are defined as follows:

$P_{1}^{r}\left(\mathbf{U}_{R}\right)=\left\{\mathbf{U} \mid \mathbf{U} \in T_{2}\left(\mathbf{U}_{R}\right)\right.$ with $\left.u>0\right\}$,

$P_{2}^{r}\left(\mathbf{U}_{R}\right)=\left\{\mathbf{U} \mid \mathbf{U}=\mathbf{J}\left(z_{L} ; \mathbf{U}_{-}, z_{R}\right)\right.$ and $\mathbf{U}_{-} \in S_{2}^{+}\left(\mathbf{U}_{L}\right)$ with $\left.\hat{u}_{R}<u_{-}<0, \overline{\hat{u}}_{R}<u<0\right\}$, $P_{3}^{r}\left(\mathbf{U}_{R}\right)=\left\{\mathbf{U} \mid \mathbf{U}=\mathbf{J}\left(z_{L} ; \mathbf{U}_{+}, z\right) ; \mathbf{U}_{+}=S_{2}^{0}\left(\mathbf{U}_{-}\right) ; \mathbf{U}_{-}=\mathbf{J}\left(z ; \mathbf{U}_{R}, z_{R}\right), z_{L} \leq z \leq z_{R}\right\}$, $P_{4}^{r}\left(\mathbf{U}_{R}\right)=\left\{\mathbf{U} \mid \mathbf{U} \in T_{2}\left(\overline{\mathbf{U}}_{R}\right)\right.$ with $\left.u<\hat{\bar{u}}_{R}\right\}$,

where $\overline{\hat{\mathbf{U}}}_{R}=\mathbf{J}\left(z_{L} ; \hat{\mathbf{U}}_{R}, z_{R}\right)$ and $\hat{\mathbf{U}}_{R}=S_{2}^{0}\left(\mathbf{U}_{R}\right)$, while $\hat{\overline{\mathbf{U}}}_{R}=S_{2}^{0}\left(\overline{\mathbf{U}}_{R}\right)$ and $\overline{\mathbf{U}}_{R}=$ $\mathbf{J}\left(z_{L} ; \mathbf{U}_{R}, z_{R}\right)$. Analogously to CASE $I_{R}$, the $\mathrm{R}-\mathrm{M}$ curve in this case is continuous and strictly increasing in the state space $(u, h+z)$.

We define

$$
u_{0 R}^{*}=\bar{u}_{R}-2 \bar{c}_{R} .
$$

Note that if $u_{0 L}>u_{0 R}^{*}$ and $h_{L}>0$, the curve $P_{1}^{l}\left(\mathbf{U}_{L}\right)$ and the $\mathrm{R}-\mathrm{M}$ curve always have an intersection point. If the intersection point lies on $P_{2}^{r}\left(\mathbf{U}_{R}\right)$, the solution has the wave configuration $A^{T}$. This is the same as for the solution related to the segment 



Fig. 34. Left: The water free surface $h+z$ at $t=0.15$. Right: The Froude number. The Riemann initial data are $\left(z_{L}, h_{L}, u_{L}\right)=$ $(0.0,0.1,2.0)$ when $x<0.5$ and $\left(z_{R}, h_{R}, u_{R}\right)=(0.2,0.177,1.69)$ when $x>0.5$.
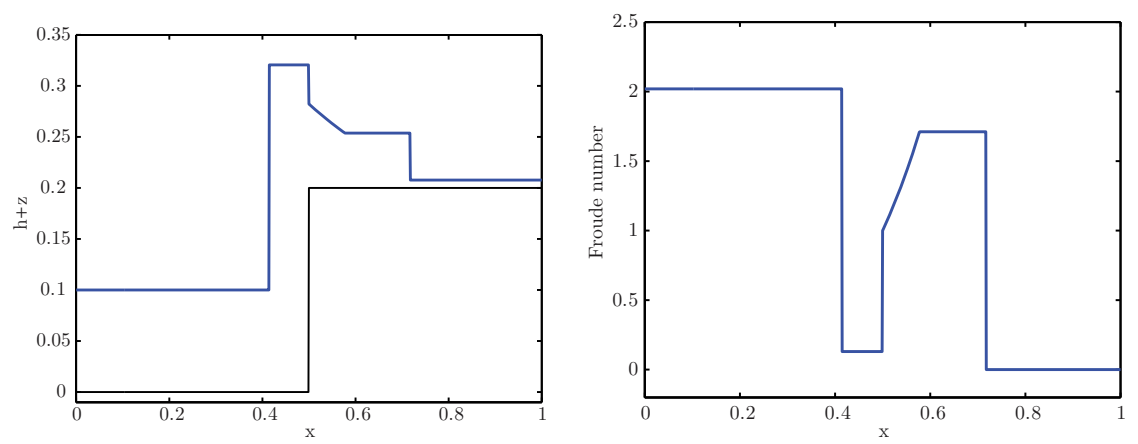

FIG. 35. Left: The water free surface $h+z$. Right: The Froude number at $t=0.15$. The Riemann initial data are $\left(z_{L}, h_{L}, u_{L}\right)=$ $(0.0,0.1,2.0)$ when $x<0.5$ and $\left(z_{R}, h_{R}, u_{R}\right)=(0.2,0.0077,0.0)$ when $x>0.5$.

$P_{2}^{r}\left(\mathbf{U}_{R}\right)$ in CASE $I_{R}$. If the intersection point lies on $P_{3}^{r}\left(\mathbf{U}_{R}\right)$, the solution has the wave configuration $C^{T}$; see Figure 17. An example is shown in Figure 43. We can see that the resonant wave occurs around $x=0.5$. If the intersection point lies on $P_{4}^{r}\left(\mathbf{U}_{R}\right)$, the solution has the wave configuration $D^{T}$; see Figure 18. An example is shown in Figure 44.

Otherwise, if $u_{0 L}<u_{0 R}^{*}$ or $h_{L}>0$, the solution has the wave configuration $D_{v}^{T}$. An example with $h_{L}>0$ is shown in Figure 45. An example with $h_{L}=0$ is shown in Figure 46.

5. Algorithm for exact Riemann solutions to the shallow water equations. In this section we present an algorithm for solving the exact Riemann problem for (1.1) and (1.3) under the assumption $z_{L}<z_{R}$. For the given Riemann initial data, if $h_{L}=$ $h_{R}=0$, the solution is $h=0$ and $u=0$ for $(x, t) \in \mathbb{R} \times \mathbb{R}^{+}$. So in the following we always assume that $h_{L}=0$ and $h_{R}=0$ cannot occur. 

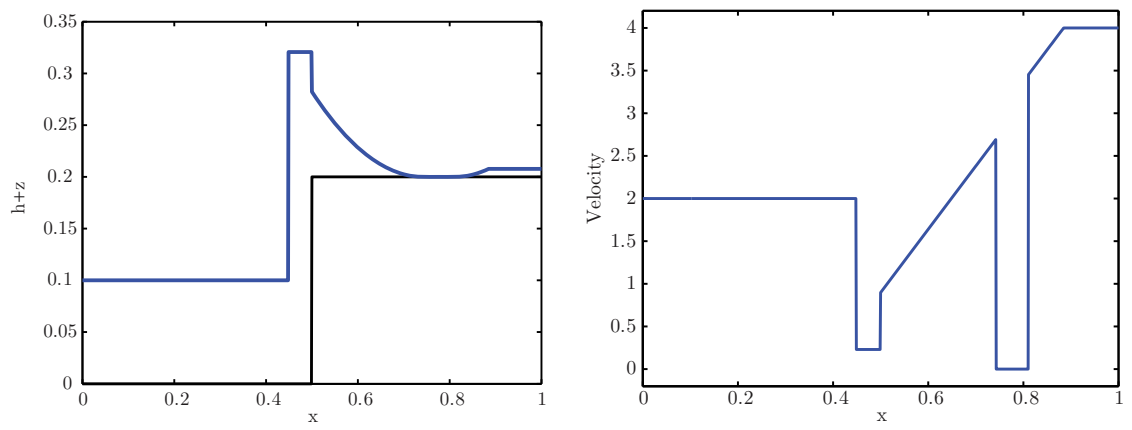

FIG. 36. Left: The water free surface $h+z$. Right: The Froude number at $t=0.09$. The Riemann initial data are $\left(z_{L}, h_{L}, u_{L}\right)=$ $(0.0,0.1,2.0)$ when $x<0.5$ and $\left(z_{R}, h_{R}, u_{R}\right)=(0.2,0.0077,4.0)$ when $x>0.5$.
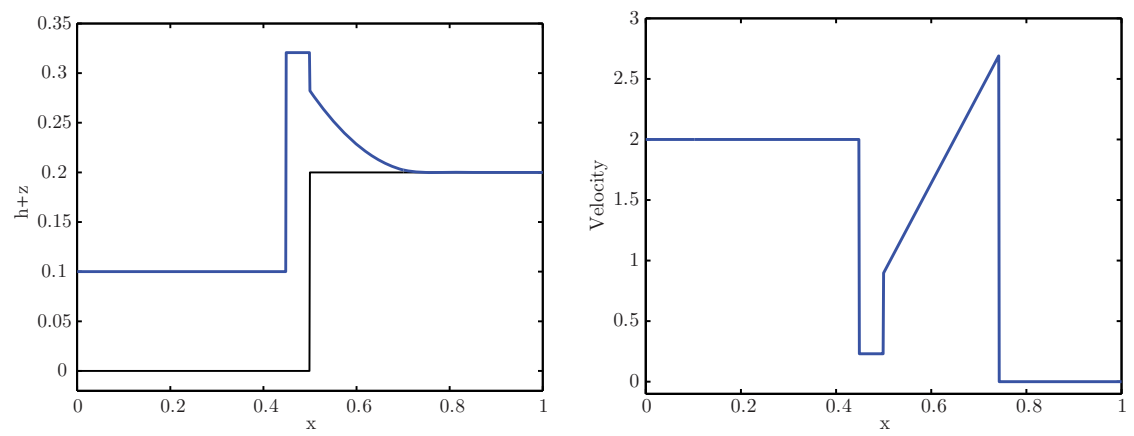

FIG. 37. Left: The water free surface $h+z$. Right: The Froudenumber at $t=0.09$. The Riemann initial data are $\left(z_{L}, h_{L}, u_{L}\right)=$ $(0.0,0.1,2.0)$ when $x<0.5$ and $\left(z_{R}, h_{R}, u_{R}\right)=(0.2,0,0)$ when $x>0.5$.

If $h_{L}=0$, there is no L-M curve. Otherwise, if $h_{L}>0$ but $u_{0 L}<0$, the $\mathrm{L}-\mathrm{M}$ curve contains only one segment which is $P_{1}^{l}\left(\mathbf{U}_{L}\right)$, defined in (4.3). Analogous to the $\mathrm{R}-\mathrm{M}$ curves, if $h_{R}=0$, there is no $\mathrm{R}-\mathrm{M}$ curve. Otherwise, if $h_{R}>0$ but $u_{0 R}>0$, the $\mathrm{R}-\mathrm{M}$ curve contains only one segment which is $P_{1}^{r}\left(\mathbf{U}_{R}\right)$, defined in (4.5). The common point of these cases is that there is no stationary wave on the $\mathrm{L}-\mathrm{M}$ and $\mathrm{R}-\mathrm{M}$ curves. Generally there are three types of possible solutions:

(1) If $h_{L}=0$ and $u_{0 R}>0$, the solution is defined in (2.27);

(2) If $h_{R}=0$ and $u_{0 L}<0$, the solution is defined in (2.25);

(3) If $u_{0 L}<0$ and $u_{0 R}>0$, the solution has the wave configuration $A_{v}$.

Besides, if $u_{O L}>0$ or $u_{0 R}<0$, the stationary wave exists except in the case when $h_{L}^{\max }+z_{L}<h_{R}^{\max }+z_{R}$ and $u_{0 R}>0$. Note that if $u_{0 R}>0$, we have $h_{R}^{\max }=0$. Therefore by (4.14) we obtain $z_{\max }<z_{R}$, i.e. CASE $I_{L}$ occurs. 

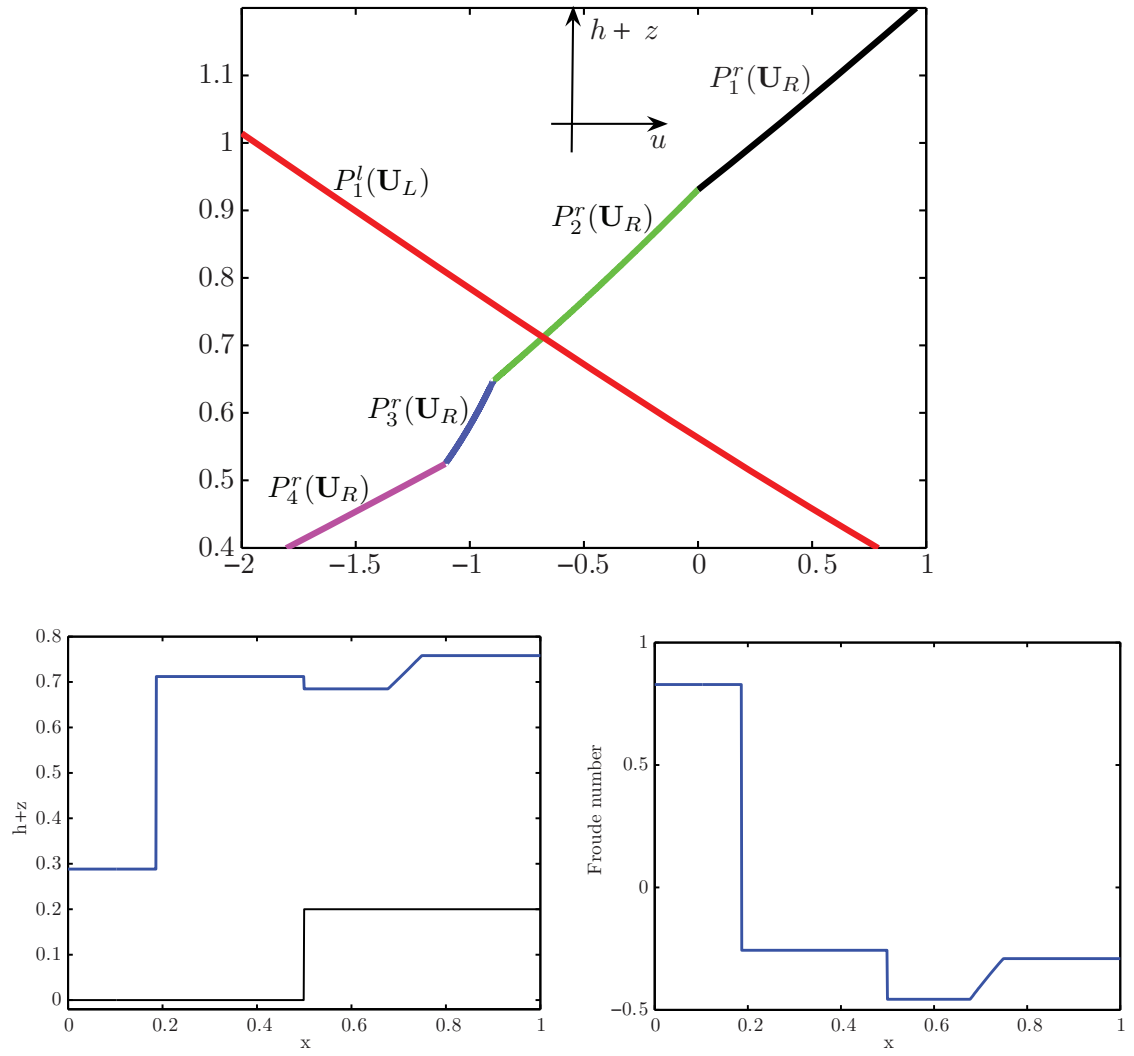

FIG. 38. Top: R-M curve $\bigcup_{k=1}^{4} P_{k}^{r}\left(\mathbf{U}_{R}\right)$. Bottom left: The water free surface $h+z$ at $t=0.15$. Bottom right: The Froude number. The Riemann initial data are $\left(z_{L}, h_{L}, u_{L}\right)=(0.0,0.2883,1.393)$ when $x<0.5$ and $\left(z_{R}, h_{R}, u_{R}\right)=(0.2,0.558,-0.68)$ when $x>0.5$.

Consider the case when the stationary wave exists. If $h_{L}^{\max }+z_{L}<h_{R}^{\max }+z_{R}$ and $u_{0 R}<0$, we have $u_{M}<0$. Hence the stationary wave is on the $\mathrm{R}-\mathrm{M}$ curve. Otherwise the stationary wave is on the $\mathrm{L}-\mathrm{M}$ curve.

According to our construction, the L-M curve is classified into 5 different cases, while the $\mathrm{R}-\mathrm{M}$ curve is classified into 2 different cases. Every case contains different types of wave configurations. Each type of wave configuration corresponds to a specific segment of the wave curve. The intermediate state $\left(h_{M}, u_{M}\right)$ of the exact Riemann solution is the intersection point of segments of the $\mathrm{L}-\mathrm{M}$ and $\mathrm{R}-\mathrm{M}$ curves. The $\mathrm{L}-\mathrm{M}$ curve, in the absence of CASES $I I I_{L}$ and $I V_{L}$ involving the bifurcation, is strictly decreasing while the $\mathrm{R}-\mathrm{M}$ curve is strictly increasing in the $(u, h+z)$ state space. This monotonicity behavior of the curves guarantees that the intersection point exists uniquely. Moreover, the L-M curve in CASES $I I I_{L}$ and $I V_{L}$ consists of more than one branch. 

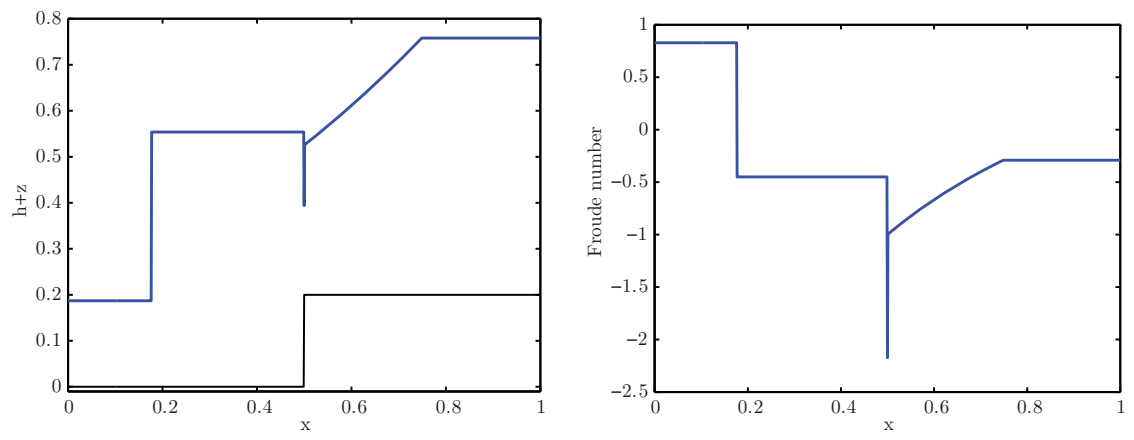

FIG. 39. Left: The water free surface $h+z$ at $t=0.15$. Right: The Froude number. The Riemann initial data are $\left(z_{L}, h_{L}, u_{L}\right)=(0.0,0.1871,1.1222)$ when $x<0.5$ and $\left(z_{R}, h_{R}, u_{R}\right)=$ $(0.2,0.558,-0.68)$ when $x>0.5$.
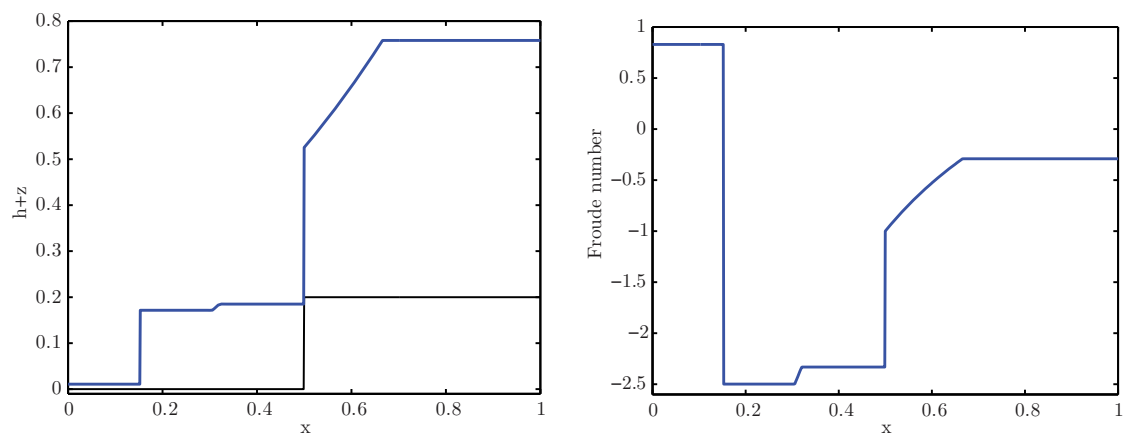

Fig. 40. Left: The water free surface $h+z$ at $t=0.1$. Right: The Froude number. The Riemann initial data are $\left(z_{L}, h_{L}, u_{L}\right)=(0.0,0.0109,0.2712)$ when $x<0.5$ and $\left(z_{R}, h_{R}, u_{R}\right)=$ $(0.2,0.558,-0.68)$ when $x>0.5$.

Every branch, however, is strictly decreasing. So every solution exists uniquely on the corresponding branch.

We present the algorithm for the exact Riemann solutions of (1.1) and (1.3) with $z_{L}<z_{R}$ in Algorithm 4 . Because of space limitations we just take the modular unit CASE $I I I_{L}$ as an example to show the algorithm for the $\mathrm{L}-\mathrm{M}$ and $\mathrm{R}-\mathrm{M}$ curves. Note that the $\mathrm{L}-\mathrm{M}$ curve in CASE $I I I_{L}$ contains bifurcation. Also the solver for the wave configuration $A$, see Algorithm 3 , is presented as an example to calculate the intermediate state $\left(u_{M}, h_{M}\right)$. The remaining cases of $\mathrm{L}-\mathrm{M}$ and $\mathrm{R}-\mathrm{M}$ curves and wave configurations can be dealt with in similar ways. The bisection method is used to solve the nonlinear system. Of course we can also adopt other iteration methods, say the secant method, to solve the problem. The Newton method is not so easy to apply because it is complicated to compute the derivative of the corresponding function. 

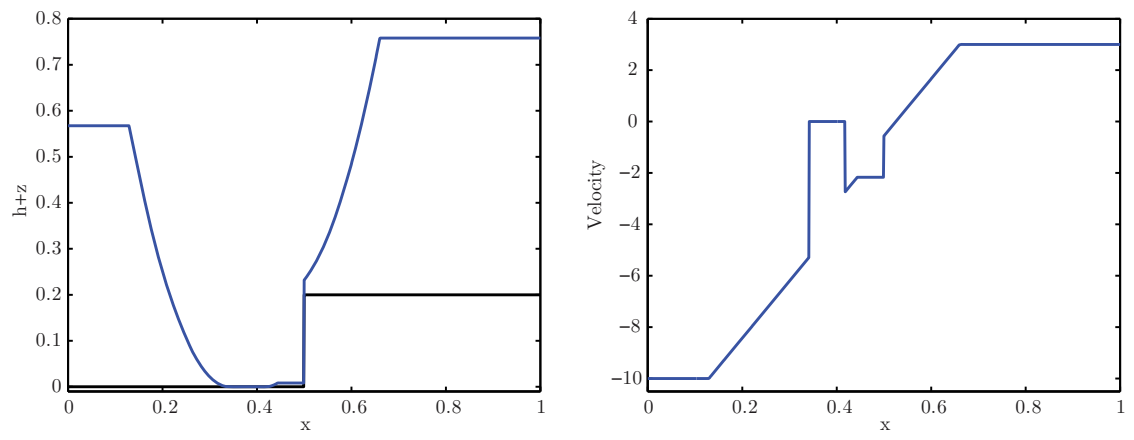

Fig. 41. Left: The water free surface $h+z$ at $t=0.03$. Right: The Froude number. The Riemann initial data are $\left(z_{L}, h_{L}, u_{L}\right)=$ $(0.0,0.5674,-10)$ when $x<0.5$ and $\left(z_{R}, h_{R}, u_{R}\right)=(0.2,0.558,3.0)$ when $x>0.5$.


FIG. 42. Left: The water free surface $h+z$ at $t=0.03$. Right: The Froude number. The Riemann initial data are $\left(z_{L}, h_{L}, u_{L}\right)=(0,0,0)$ when $x<0.5$ and $\left(z_{R}, h_{R}, u_{R}\right)=(0.1,0.558,3.0)$ when $x>0.5$.

6. Conclusion. For any given Riemann initial data $\mathbf{U}_{L}$ and $\mathbf{U}_{R}$ with $z_{L}<z_{R}$, we obtain all possible Riemann solutions to the shallow water equation by constructing the $\mathrm{L}-\mathrm{M}$ and $\mathrm{R}-\mathrm{M}$ curves. We analyze the behavior of the $\mathrm{L}-\mathrm{M}$ and $\mathrm{R}-\mathrm{M}$ curves. We observe that on one hand if the intersection points belong to CASES $I I I_{L}$ and $I V_{L}$ of the $\mathrm{L}-\mathrm{M}$ curves, a bifurcation appears on the L-M curves. There may be three possible solutions due to the bifurcation. In the other cases, the solution always uniquely exists. The dry bed problem has also been considered in this framework. Here the dry bed problem refers to two subcases. One is for the water propagating to a dry bed, i.e. $h_{L}=0$ or $h_{R}=0$. The other one is for the dry bed state emerging due to the motion of the flow. 

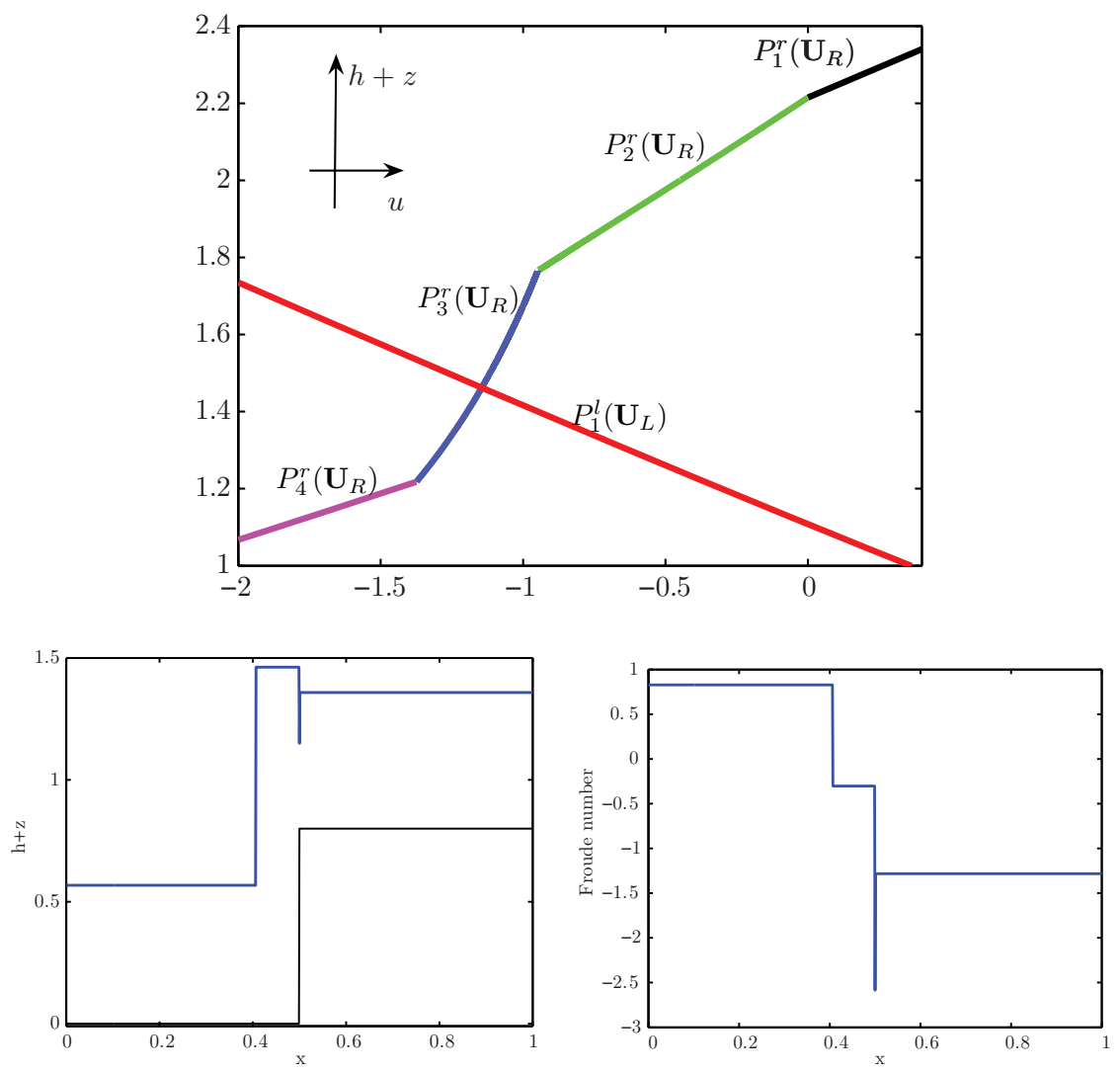

Fig. 43. Top: R-M curve $\bigcup_{k=1}^{4} P_{k}^{r}\left(\mathbf{U}_{R}\right)$. Bottom left: The water free surface $h+z$. Bottom right: The Froude number at $t=0.03$. The Riemann initial data are $\left(z_{L}, h_{L}, u_{L}\right)=(0.0,0.5674,1.9542)$ when $x<0.5$ and $\left(z_{R}, h_{R}, u_{R}\right)=(0.8,0.558,-3.0)$ when $x>0.5$. 



FIG. 44. Left: The water free surface $h+z$. Right: The Froude number at $t=0.01$. The Riemann initial data are $\left(z_{L}, h_{L}, u_{L}\right)=(0.0,0.0109,0.2712)$ when $x<0.5$ and $\left(z_{R}, h_{R}, u_{R}\right)=$ $(0.8,0.558,-3.0)$ when $x>0.5$.
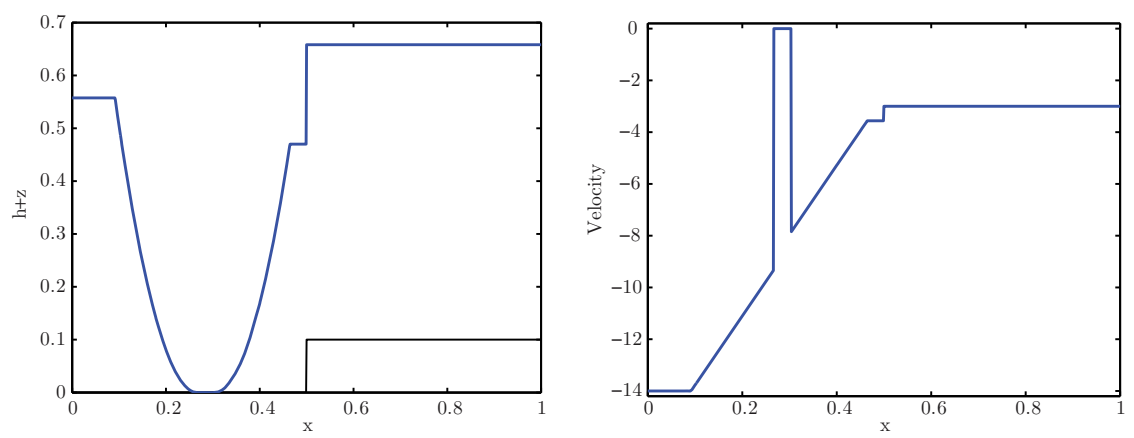

FIG. 45. Left: The water free surface $h+z$ at $t=0.025$. Right: The Froude number. The Riemann initial data are $\left(z_{L}, h_{L}, u_{L}\right)=(0.0,0.5574,-14.0)$ when $x<0.5$ and $\left(z_{R}, h_{R}, u_{R}\right)=$ $(0.1,0.558,-3.0)$ when $x>0.5$.
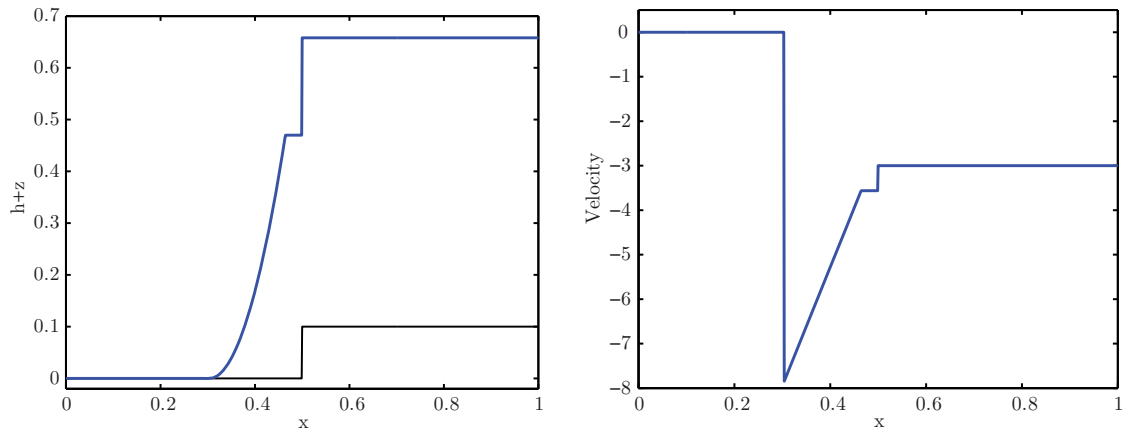

FIG. 46. Left: The water free surface $h+z$ at $t=0.025$. Right: The Froude number. The Riemann initial data are $\left(z_{L}, h_{L}, u_{L}\right)=(0,0,0)$ when $x<0.5$ and $\left(z_{R}, h_{R}, u_{R}\right)=(0.1,0.558,-3.0)$ when $x>0.5$. 


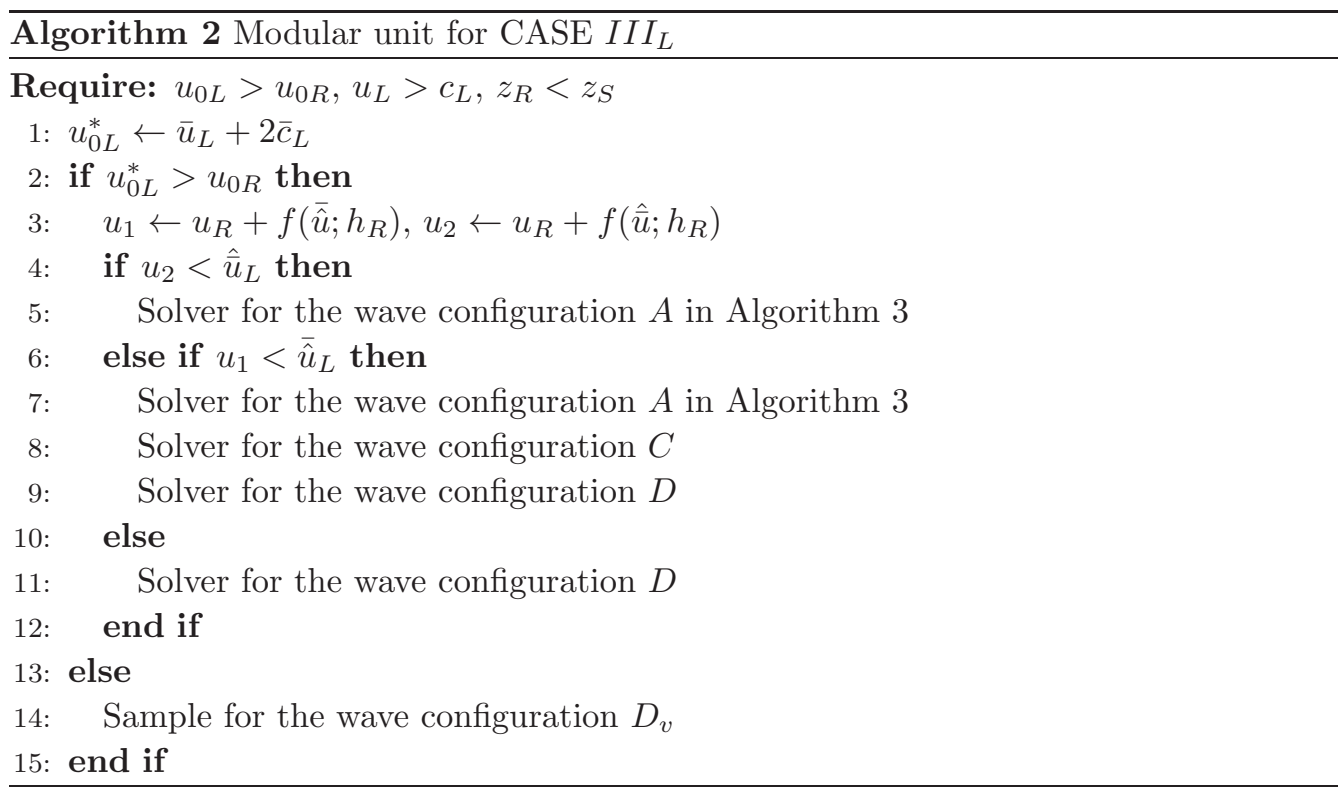




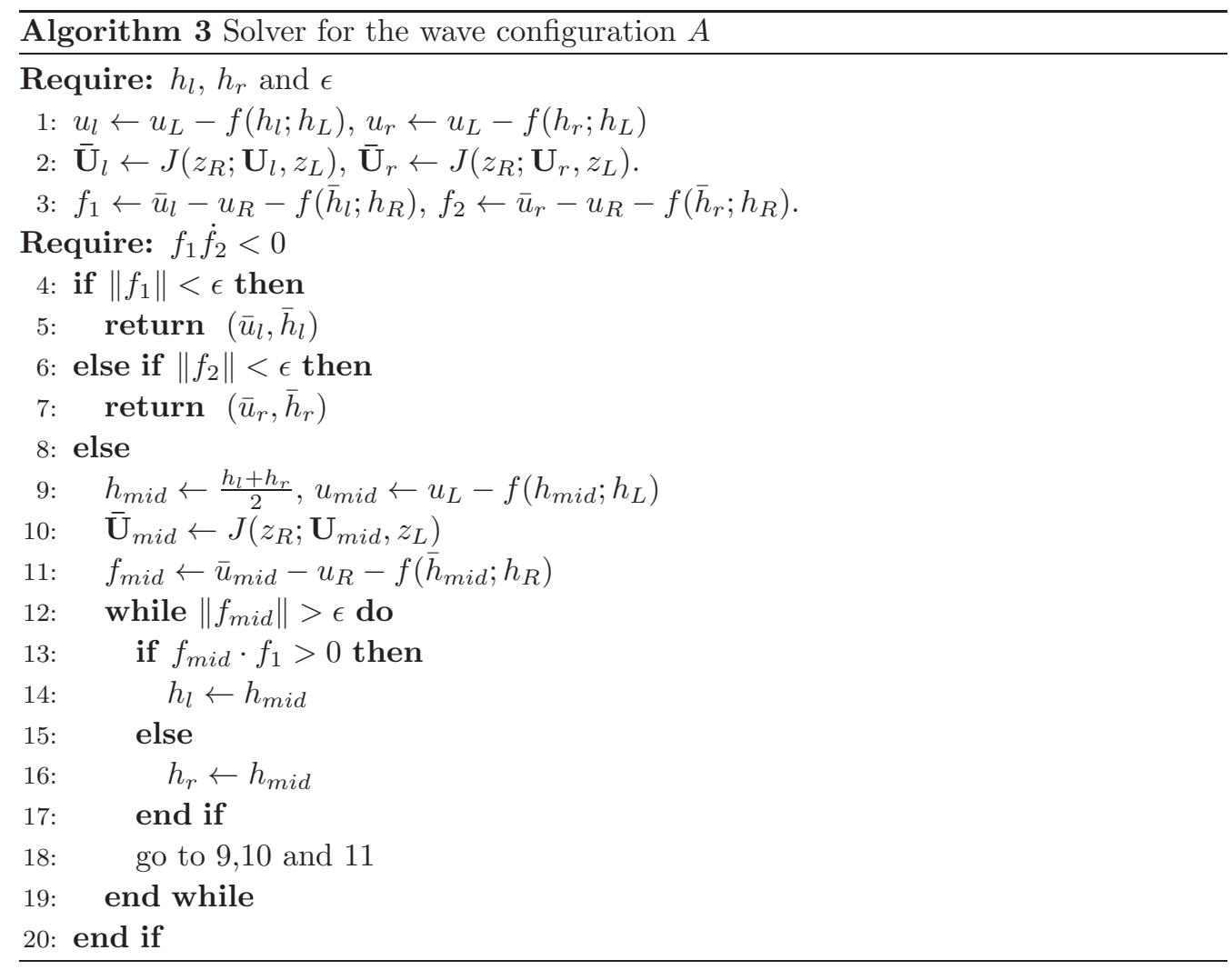




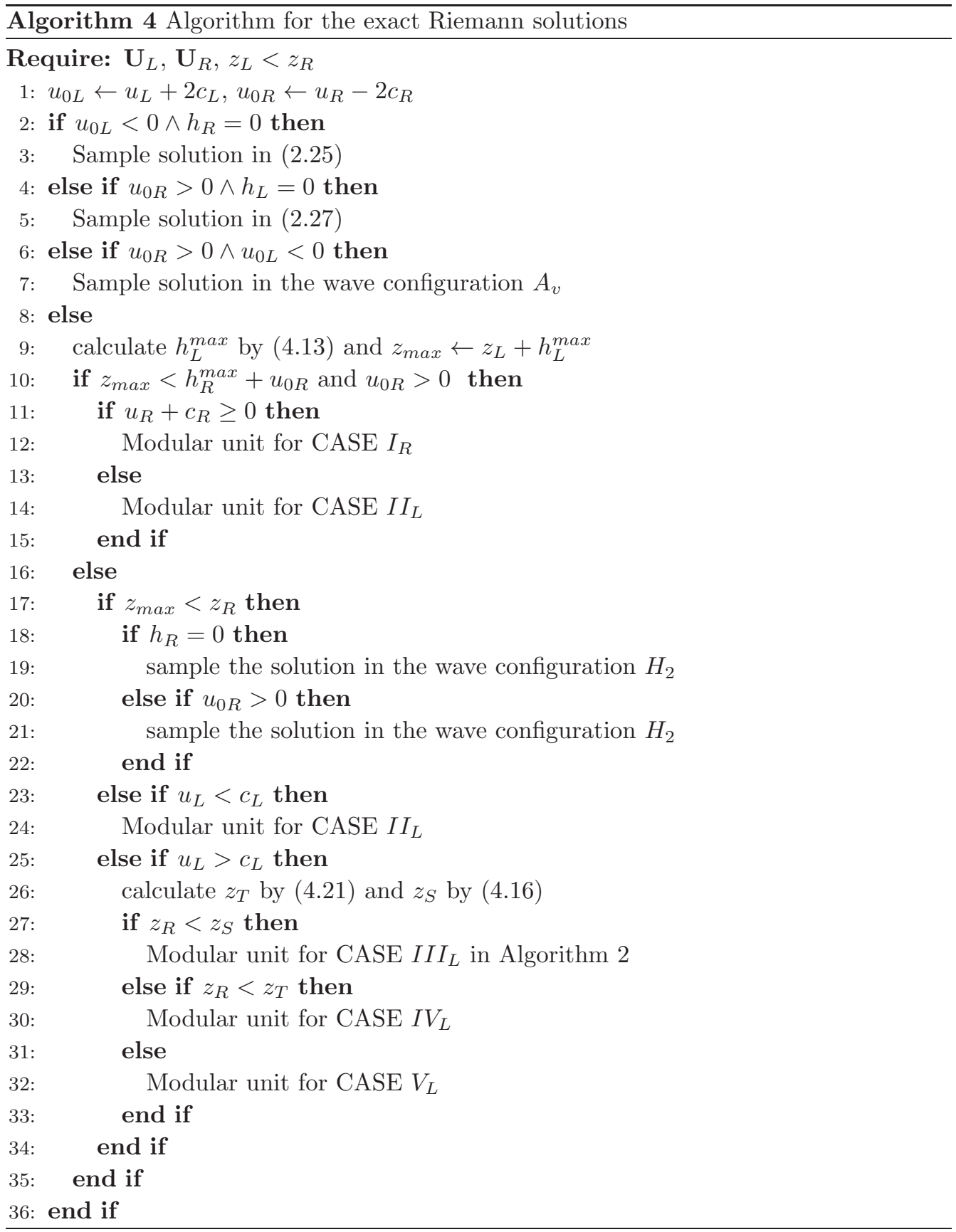




\section{REFERENCES}

[1] Ashwin Chinnayya, Alain-Yves LeRoux, and Nicolas Seguin, A well-balanced numerical scheme for the approximation of the shallow-water equations with topography: The resonance phenomenon, Int. J. Finite Vol. 1 (2004), no. 1, 33. MR2465450 (2009j:65186)

[2] J.J. Stoker, Water Waves, Interscience,. New York, 1957.

[3] Nikolai Andrianov, Performance of numerical methods on the non-unique solution to the Riemann problem for the shallow water equations, Internat. J. Numer. Methods Fluids 47 (2005), no. 8-9, 825-831, DOI 10.1002/fld.846. MR2123795(2005i:76079)

[4] Nikolai Andrianov and Gerald Warnecke, The Riemann problem for the Baer-Nunziato twophase flow model, J. Comput. Phys. 195 (2004), no. 2, 434-464, DOI 10.1016/j.jcp.2003.10.006. MR 2046106(2004m:76165)

[5] Francisco Alcrudo and Fayssal Benkhaldoun, Exact solutions to the Riemann problem of the shallow water equations with a bottom step, Comput. \& Fluids 30 (2001), no. 6, 643-671, DOI 10.1016/S0045-7930(01)00013-5. MR1859269 (2002g:76012)

[6] F. Benkhaldoun and L. Quivy, A Non Homogeneous Riemann Solver for shallow water and two phase flows, Flow, Turbulence and Combustion 76 (2006), pp. 391-402.

[7] R. Bernetti, V. A. Titarev, and E. F. Toro, Exact solution of the Riemann problem for the shallow water equations with discontinuous bottom geometry, J. Comput. Phys. 227 (2008), no. 6, 32123243, DOI 10.1016/j.jcp.2007.11.033. MR.2392731(2009a:76015)

[8] R. W. D. Nickalls, A New Approach to Solving the Cubic: Cardan's Solution Revealed, The Mathematical Gazette, 77 (Nov., 1993), pp. 354-359.

[9] E. Han, M. Hantke, and G. Warnecke, Exact Riemann solutions in ducts with discontinuous crosssection, J. Hyp. Diff. Equations 9 (2012), pp. 403-449.

[10] Jiequan Li and Guoxian Chen, The generalized Riemann problem method for the shallow water equations with bottom topography, Internat. J. Numer. Methods Engrg. 65 (2006), no. 6, 834-862, DOI 10.1002/nme.1471. MR2200026(2006j:76022)

[11] E. F. Toro, The Dry-Bed Problem in Shallow-Water Flows, College of Aeronautics Reports, 1990.

[12] E. F. Toro, Shock-Capturing Methods for Free-Surface Shallow Flows, Wiley and Sons. Ltd., 2001.

[13] Eli Isaacson and Blake Temple, Nonlinear resonance in systems of conservation laws, SIAM J. Appl. Math. 52 (1992), no. 5, 1260-1278, DOI 10.1137/0152073. MR1182123 (93f:35140)

[14] Jihwan Kim and Randall J. LeVeque, Two-layer shallow water system and its applications, Hyperbolic problems: Theory, numerics and applications, Proc. Sympos. Appl. Math., vol. 67, Amer. Math. Soc., Providence, RI, 2009, pp. 737-743. MR 2605269 (2011e:76024)

[15] Paola Goatin and Philippe G. LeFloch, The Riemann problem for a class of resonant hyperbolic systems of balance laws (English, with English and French summaries), Ann. Inst. H. Poincaré Anal. Non Linéaire 21 (2004), no. 6, 881-902, DOI 10.1016/j.anihpc.2004.02.002. MR2097035 (2006i:35225)

[16] V. V. Ostapenko, Dam-break flows over a bottom step (Russian, with Russian summary), Prikl. Mekh. Tekhn. Fiz. 44 (2003), no. 4, 51-63, DOI 10.1023/A:1024292822989; English transl., J. Appl. Mech. Tech. Phys. 44 (2003), no. 4, 495-505. MR2009465 (2004e:76026)

[17] D. Rochette, S. Clain, and W. Bussière, Unsteady compressible flow in ducts with varying crosssection: Comparison between the nonconservative Euler system and axisymmetric flow model. Preprint submitted.

[18] Philippe G. LeFloch, Kinetic relations for undercompressive shock waves. Physical, mathematical, and numerical issues, Nonlinear partial differential equations and hyperbolic wave phenomena, Contemp. Math., vol. 526, Amer. Math. Soc., Providence, RI, 2010, pp. 237-272, DOI 10.1090/conm/526/10384. MR2731995 (2012d:35222)

[19] Philippe G. LeFloch and Mai Duc Thanh, The Riemann problem for the shallow water equations with discontinuous topography, Commun. Math. Sci. 5 (2007), no. 4, 865-885. MR2375051 (2009k:35179)

[20] Philippe G. LeFloch and Mai Duc Thanh, A Godunov-type method for the shallow water equations with discontinuous topography in the resonant regime, J. Comput. Phys. 230 (2011), no. 20, 76317660, DOI 10.1016/j.jcp.2011.06.017. MR2823568 (2012g:76028)

[21] D. Marchesin and P. J. Paes-Leme, A Riemann problem in gas dynamics with bifurcation: Hyperbolic partial differential equations, III, Comput. Math. Appl. Part A 12 (1986), no. 4-5, 433-455. MR841979 (87e:76106) 